Library, N.W. Bldg

FEB 11965

NBS MONOGRAPH 79

On the Statistical Theory of

Electromagnetic Waves in a

Fluctuating Medium (II)

Mathematical Basis of the

Analogies to Quantum Field Theory

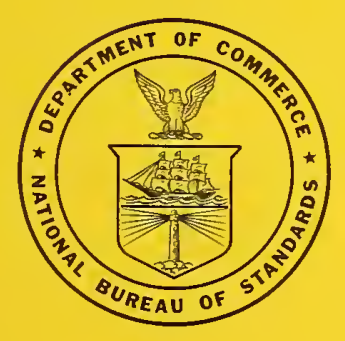

U.S. DEPARTMENT OF COMMERCE

NATIONAL BUREAU OF STANDARDS 


\section{THE NATIONAL BUREAU OF STANDARDS}

The National Bureau of Standards is a principal focal point in the Federal Government for assuring maximum application of the physical and engineering sciences to the advancement of technology in industry and commerce. Its responsibilities include development and maintenance of the national standards of measurement, and the provisions of means for making measurements consistent with those standards; determination of physical constants and properties of materials; development of methods for testing materials, mechanisms, and structures, and making such tests as may be necessary particularly for government agencies; cooperation in the establishment of standard practices for incorporation in codes and specifications; advisory service to government agencies on scientific and technical problems; invention and development of devices to serve special needs of the Government; assistance to industry, business, and consumers in the development and acceptance of commercial standards and simplified trade practice recommendations; administration of programs in cooperation with United States business groups and standards organizations for the development of international standards of practice; and maintenance of a clearinghouse for the collection and dissemination of scientific, technical, and engineering information. The scope of the Bureau's activities is suggested in the following listing of its four Institutes and their organizational units.

Institute for Basic Standards. Electricity. Metrology. Heat. Radiation Physics. Mechanics. Applied Mathematics. Atomic Physics. Physical Chemistry. Laboratory Astrophysics. ${ }^{*}$ Radio Standards Laboratory: Radio Standards Physics; Radio Standards Engineering. ${ }^{*}$ Office of Standard Reference Data.

Institute for Materials Research. Analytical Chemistry. Polymers. Metallurgy. Inorganic Materials. Reactor Radiations. Cryogenics.** Office of Standard Reference Materials.

Central Radio Propagation Laboratory.** Ionosphere Research and Propagation. Troposphere and Space Telecommunications. Radio Systems. Upper Atmosphere and Space Physics.

Institute for Applied Technology. Textiles and Apparel Technology Center. Building Research. Industrial Equipment. Information Technology. Performance Test Development. Instrumentation. Transport Systems. Office of Technical Services. Office of Weights and Measures. Office of Engineering Standards. Office of Industrial Services.

\footnotetext{
*NBS Group, Joint Institute for Laboratory Astrophysics at the University of Colorado.

** Located at Boulder, Colorado.
} 
UNITED STATES DEPARTMENT OF COMMERCE - Luther H. Hodges, Secretary NATIONAL BUREAU OF STANDARDS - A. V. Astin, Director

\title{
On the Statistical Theory of Electromagnetic Waves in a Fluctuating Medium(II)
}

\section{Mathematical Basis of the Analogies to Quantum Field Theory}

\author{
K. Furutsu \\ Central Radio Propagation Laboratory \\ National Bureau of Standards \\ Boulder, Colo.
}

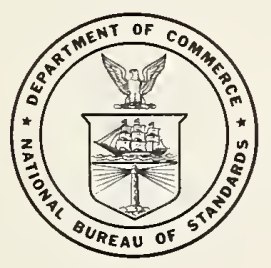

National Bureau of Standards Monograph 79 Issued December 7, 1964 
Library of Congress Catalog Card Number : 64-60057 


\title{
On the Statistical Theory of Electromagnetic Waves in a Fluctuating Medium (II). Mathematical Basis of the Analogies to Quantum Field Theory*
}

\author{
K. Furutsu \\ National Bureau of Standards, Boulder, Colo.
}

\begin{abstract}
Many analogies to quantum field theory are inherent in the statistical theory of waves. This is due to the fact that basic equations exist in the latter theory which correspond closely to the fundamental equations of the former theory; i.e., to the commutation relations and the Heisenberg equation of motion. A probability density function of waves is introduced here which corresponds to the probability amplitude function in quantum mechanies. The boundary conditions at infinity for this probability density function are then found to be expressed in the same form as the vacuum boundary conditions in field theory. The theory of the statistical Green's functions and their relationships to the expectation values of the physical variables is also extensively developed, using auxiliary external sources of the wave and of the fluctuating medium. It is found that there exists a one-to-one correspondence between the formalism of Green's functions presented here and that used in field theory. The above correspondence may be important for a further development of the statistical theory of waves, just as the advanced techniques of field theory have greatly influenced the development of thermodynamics (or statistical physics).
\end{abstract}

\section{1.. Introduction}

In recent years, important advances have been carried out in the field of statistical physics by a successful application of well-developed methods of quantum field theory to the study of systems consisting of a large number of interacting particles which are in thermodynamic equilibrium [Alekseev, 1961; Bardeen, Cooper, and Schrieffer, 1957; Matsubara, 1955; Martin and Schwinger, 1959]. Especially, the method of thermodynamic Green's functions has been extensively used in the study of important problems of statistical physics, and in principle, all the necessary physical observables seem to have been obtained in terms of the many particle Green's functions.

On the other hand, the situation is similar also in the statistical theory of ordinary waves in a fluctuating medium, as is emphasized in part I of this series of papers [Furutsu, 1963]. ${ }^{1}$ In fact, all the necessary information about the statistical system of waves can be obtained in terms of the manifold Green's functions. Let $\psi\left(x_{1}, x_{2}\right)$ be the single Green's function as defined by (I.2.14) and $\psi_{0}\left(x_{1}, x_{2}\right)$ be the corresponding function in the unperturbed medium; then $\psi\left(x_{1}, x_{2}\right)$ is generally the solution of an integral equation of the form

$$
\psi\left(x_{1}, x_{2}\right)=\psi_{0}\left(x_{1}, x_{2}\right)+\int(d x) \psi_{0}\left(x_{1}, x\right) \delta k(x) \psi\left(x, x_{2}\right),
$$

where $\delta k(x)=\gamma \phi(x)$ is the fluctuating part of $k(x)$ representing the medium constant, and $\gamma$ and $\phi(x)$ are a constant matrix and a scalar function having the vanishing average $\langle\phi(x)\rangle=0$, respectively. The expectation value $\left\langle\psi\left(x_{1}, x_{2}\right)\right\rangle \equiv G\left(x_{1}, x_{2}\right)$ is formally obtained by expanding (1.1) in a Born series,

$$
\begin{aligned}
\psi\left(x_{1}, x_{2}\right)=\psi_{0}\left(x_{1}, x_{2}\right)+\int(d x) \psi_{0}\left(x_{1}, x\right) \gamma \phi(x) \psi_{0}\left(x, x_{2}\right) \\
\quad+\iint(d x)\left(d x^{\prime}\right) \psi_{0}\left(x_{1}, x\right) \gamma \phi(x) \psi_{0}\left(x, x^{\prime}\right) \gamma \phi\left(x^{\prime}\right) \psi_{0}\left(x^{\prime}, x_{2}\right)+\ldots
\end{aligned}
$$

and taking the average value for each term of this series. Similarly, the dual Green's function, $G\left(x_{1}, x_{2} \mid x_{1}^{\prime}, x_{2}^{\prime}\right) \equiv\left\langle\psi\left(x_{1}, x_{1}^{\prime}\right) \psi\left(x_{2}, x_{2}^{\prime}\right)\right\rangle$, is obtained as the product of the series similar to (1.2), averaged

*The theory is developed in three dimensional space.

Part I appeared in J. Res. NBS 67D (Radio Prop.) No. 3, 303-323 (May-June 1963).

${ }^{1}$ Reference to equations in the work will be written in the typical form (I.2.14). Added in proof: Recently, R. C. Bourret kindly notified the author of his recent paper [1962] in which he also suggested a strong correspondence with field theory by several examples; Nuovo Cimento, XXVI, 1. 
over all possible values of $\phi(x)$. Thus the higher-order Green's functions can be obtained in the same way. Especially, when the fluctuation follows the gaussian multivariate normal distribution, it is well known [Middleton, 1960] that

$$
\left\langle\phi\left(x_{1}\right) \phi\left(x_{2}\right) \ldots \phi\left(x_{2 n}\right)\right\rangle=\Sigma_{p}\left\langle\phi\left(x_{1}\right) \phi\left(x_{2}\right)\right\rangle \ldots\left\langle\phi\left(x_{2 n-1}\right) \phi\left(x_{2 n}\right)\right\rangle,\left\langle\phi\left(x_{1}\right) \phi\left(x_{2}\right) \ldots \phi\left(x_{2 n+1}\right)\right\rangle=0
$$

where $\Sigma_{p}$ stands for the sum over all the terms obtained from all possible combinations of different pairs composing the set of variables $x_{1}, x_{2}, \ldots, x_{2 n}$.

On the other hand, the necessary and sufficient description of the whole statistical system of waves can be obtained only after obtaining the full set of these statistical Green's functions of infinite number. Their knowledge is proven to be equivalent to that of the probability distribution function of waves, defined such that the probability that the wave has particular values between $\psi(x)^{\prime}$ and $\psi(x)^{\prime}+d \psi(x)^{\prime}$ in the whole space $\Sigma$ is given by

$$
d \psi[\Sigma]^{\prime}\left\langle\psi[\Sigma]^{\prime} \mid\right\rangle,
$$

where $\psi[\Sigma]^{\prime}$ stands for the values $\psi(x)^{\prime}$ at all points in $\Sigma$ : it means that, if all points in space are represented by a discrete set of points $x_{1}, x_{2}, \ldots, x_{N}$ of finite number, assuming a scalar real value of $\psi(x)$ for the moment, (1.4) represents (appendix 7)

$$
d \psi[\Sigma]^{\prime}\left\langle\psi[\Sigma]^{\prime} \mid\right\rangle \equiv d \psi\left(x_{1}\right)^{\prime} d \psi\left(x_{2}\right)^{\prime} \ldots . d \psi\left(x_{N}\right)^{\prime}\left\langle\psi\left(x_{1}\right)^{\prime}, \psi\left(x_{2}\right)^{\prime}, \ldots, \psi\left(x_{N}\right)^{\prime} \mid\right\rangle .
$$

In the Fourier representation,

$$
\begin{aligned}
\left\langle\psi\left(x_{1}\right)^{\prime}, \psi\left(x_{2}\right)^{\prime}, \ldots, \psi\left(x_{N}\right)^{\prime} \mid\right\rangle & =\left(\frac{I}{2 \pi}\right)^{N} \int_{-\infty}^{\infty} d \lambda_{1} \int_{-\infty}^{\infty} d \lambda_{2} \ldots \int_{-\infty}^{\infty} d \lambda_{N} \\
\times & \exp \left[i\left\{\lambda_{1} \psi\left(x_{1}\right)^{\prime}+\lambda_{2} \psi\left(x_{2}\right)^{\prime}+\ldots .+\lambda_{N} \psi\left(x_{N}\right)^{\prime}\right\}\right]\left\langle\lambda_{1}, \lambda_{2}, \ldots, \lambda_{N} \mid\right\rangle,
\end{aligned}
$$

it is proven that all the coefficients of the Taylor series of $\left.\left\langle\lambda_{1}, \lambda_{2}, \ldots ., \lambda_{N}\right\rangle\right\rangle$ can be given in terms of the statistical Green's functions. In fact, assuming the excitation of the wave by an external source $j(x)$ :

$$
\psi(x)=\int_{\Sigma} \psi\left(x, x^{\prime}\right) j\left(x^{\prime}\right)\left(d x^{\prime}\right)
$$

the inverse Fourier transformation of (1.6) yields

$$
\begin{gathered}
\left\langle\lambda_{1}, \lambda_{2}, \ldots, \lambda_{N} \mid\right\rangle=\sum_{n_{1}, n_{2}, \ldots, n_{N}=0}^{\infty} \frac{\left(-i \lambda_{1}\right)^{n_{1}}\left(-i \lambda_{2}\right)^{n_{2}} \ldots\left(-i \lambda_{N}\right)^{n_{N}}}{n_{1} ! n_{2} ! \ldots n_{N} !} \\
\times\left\langle\left\{\psi\left(x_{1}\right)\right\}^{n_{1}}\left\{\psi\left(x_{2}\right)\right\}^{n_{2}} \ldots .\left\{\psi\left(x_{N}\right)\right\}^{n_{N}}\right\rangle,
\end{gathered}
$$

where $\psi\left(x_{j}\right)$ (without prime) is given by (1.7) and generally

$$
\begin{aligned}
\left\langle\psi\left(y_{1}\right) \psi\left(y_{2}\right) \ldots \psi\left(y_{m}\right)\right\rangle=\int\left(d y_{1}^{\prime}\right) \int & \left(d y_{2}^{\prime}\right) \ldots \int\left(d y_{m}^{\prime}\right) \\
& \times G\left(y_{1}, y_{2}, \ldots, y_{m} \mid y_{1}^{\prime}, y_{2}^{\prime}, \ldots, y_{m}^{\prime}\right) j\left(y_{1}^{\prime}\right) j\left(y_{2}^{\prime}\right) \ldots . j\left(y_{m}^{\prime}\right),
\end{aligned}
$$

in terms of the Green's functions

$$
G\left(y_{1}, y_{2}, \ldots, y_{m} \mid y_{1}^{\prime}, y_{2}^{\prime}, \ldots, y_{m}^{\prime}\right)=\left\langle\psi\left(y_{1}, y_{1}^{\prime}\right) \psi\left(y_{2}, y_{2}^{\prime}\right) \ldots \psi \psi\left(y_{m}, y_{m}^{\prime}\right)\right\rangle
$$


The above results are the same even if the wave function is a complex vector; the independent variables, corresponding to the above scalar $\psi(x)$, are to be prepared for each component and also for each real and imaginary part.

When evaluating the Green's functions of (1.9), we may use the Born series expansion (1.2), together with (1.3), or other corresponding rule which purely depends on the prescribed distribution function of the medium fluctuation. Thus, especially when the fluctuation has a gaussian multivariate normal distribution so that (1.3) is applicable, the diagram method can be used almost in the same way as in the field theory (part I); i.e., in the integrand of each term of the series thus obtained, every factor of $\psi_{0}\left(x, x^{\prime}\right)$ can be represented by a solid line on a sheet of paper connecting the points $x$ and $x^{\prime}$, and every factor of $\left\langle\phi(x) \phi\left(x^{\prime}\right)\right\rangle$ can be represented by a dotted line connecting the points $x$ and $x^{\prime}$, and the final result is obtained by summing up the contributions from all the possible graphs in the given order of approximation. Thus, in the same way as in the field theory, we would have the self-energy parts of a graph, to each of which only two solid lines are connected, and the vertex parts of a graph to each of which two solid lines and one dotted line are connected, etc. Thus, the same techniques as used in the field theory [Dyson, 1949a, 1949b] are available to treat these parts of a graph (theory of renormalization), as is done in part $\mathrm{I}^{2}$

Here a question might arise: why are there such close analogies between the statistical theory of waves and the quantum field theory? The Feynman diagram technique is a due consequence of the quantization of field, the Heisenberg equation of motion, and the boundary (vacuum) condition at infinity. If the expectation values of all the physical observables in the field theory can be expressed in terms of Green's functions, we ask whether, inversely, the commutation relations and the equation of motion, which are the fundamental bases of the quantum field theory, could be derived from the knowledge of the Green's functions. It may be possible [Schwinger, 19511954], and the basic equations to be used for the proof will be those equations corresponding (though not exactly) to (1.6) and (1.8) which give the connection between the Green's functions and the probability density function of the waves. The latter function apparently corresponds to the pobability amplitude function in quantum mechanics, which satisfies the Schrödinger equation. In this equation, all the physical variables are represented by matrices or operators which operate on the probability amplitude function, and the equation prescribes the time variation of the probability amplitude function. Thus, it implies the complete description of the quantum field theory.

The major purpose of this Monograph is to show the existence of fundamental equations in the statistical theory of waves that correspond to the commutation relations in quantum mechanics between the physical variables involved, and also to the Schrödinger equation (or the Heisenberg equation of motion in the Heisenberg picture). As a consequence, many analogies to the field theory are obtained. These fundamental relationships between the two theories will be worth while since other useful techniques used in the field theory, such as unperturbational methods not based on Born series, may then be available also for the statistical theory of waves.

In this Monograph, the distribution of the electromagnetic wave on a plane is represented in terms of a complete set of orthogonal modes of waves by the use of a method which is essentially the same as the one Marcuvitz and Schwinger [1951] used for the representation of the electromagnetic Green's function. A probability density function of waves is then introduced, which is somewhat different from that defined in (1.4) and (1.5), to describe the statistical system of the waves in a fluctuating medium. A major effort is made to find the equation to be satisfied by the probability density function, followed by an inevitable introduction of matrix representations of the physical variables involved; the boundary conditions at infinity are examined next. The Green's functions are extensively discussed, together with their relationships to the expectation values of physical observables.

The standard notations used in quantum mechanics are employed here unless otherwise specially indicated. ${ }^{3}$

\footnotetext{
${ }^{2}$ A similar diagram method is adopted in the theory of scattering by impurities in metals by Rickayzen [1961].

${ }^{3}$ For example, P. A. M. Dirac, The Principle of Quantum Mechanics, Oxford Press (1958).
} 


\section{Wave Equation and Representation of the Wave Function}

In this Monograph, Maxwell's equations are used in a symbolic form, and the following notations are employed: Latin subscripts assume values ranging from 1 to 3 , and a repeated index is to be so summed. The coordinate vector in space is denoted by $x_{i}=(x)$.

Using the conventional notations, Maxwell's equations are given in gaussian units (time factor $\left.e^{i \omega t}\right)$ by

$$
\operatorname{rot} H-i(\omega / c) \epsilon E=4 \pi \mathrm{I} / c, \operatorname{rot} E+i(\omega / c) \mu H=0 .
$$

Introducing the matrices

$$
s_{1}=\left(\begin{array}{rrr}
0 & 0 & 0 \\
0 & 0 & i \\
0 & -i & 0
\end{array}\right), \quad s_{2}=\left(\begin{array}{rrr}
0 & 0 & -i \\
0 & 0 & 0 \\
i & 0 & 0
\end{array}\right), \quad s_{3}=\left(\begin{array}{rrr}
0 & i & 0 \\
-i & 0 & 0 \\
0 & 0 & 0
\end{array}\right),
$$

the operator rot as a three-rowed matrix is expressed by

$$
r o t=s_{i} \partial_{i}=(s \partial), \quad \partial_{j}=i \frac{\partial}{\partial x_{j}} \quad(j=1,2,3) .
$$

Thus, Maxwell's equations can be expressed in the form

$$
(s \partial)\left(\begin{array}{ll}
0 & 1 \\
1 & 0
\end{array}\right)\left(\begin{array}{l}
i E \\
H
\end{array}\right)-\frac{\omega}{c}\left(\begin{array}{ll}
\epsilon & 0 \\
0 & \mu
\end{array}\right)\left(\begin{array}{l}
i E \\
H
\end{array}\right)=\left(\begin{array}{c}
\frac{4 \pi}{c} I \\
0
\end{array}\right),
$$

which, on using the two-rowed Pauli's matrices,

$$
\rho_{1}=\left(\begin{array}{ll}
0 & 1 \\
1 & 0
\end{array}\right), \quad \rho_{2}=\left(\begin{array}{rr}
0 & -i \\
i & 0
\end{array}\right), \quad \rho_{3}=\left(\begin{array}{rr}
1 & 0 \\
0 & -1
\end{array}\right),
$$

takes the form

$$
\left[\rho_{1}(s \partial)-k\right] \psi=\eta
$$

or

$$
\left[\gamma_{i} \partial_{i}-k\right] \psi=\eta, \quad \gamma_{i}=\rho_{1} s_{i} .
$$

Here

$$
\psi=\left(\begin{array}{c}
i E \\
H
\end{array}\right) \text { and } \eta=\left(\begin{array}{c}
\frac{4 \pi}{c} I \\
0
\end{array}\right)
$$

are one-column matrices of 6 elements, and

$$
k=\frac{\omega}{2 c}\left\{\left(1+\rho_{3}\right) \epsilon+\left(1-\rho_{3}\right) \mu\right\},
$$

a $6 \times 6$ matrix, is Hermitian when the medium is lossless. This lossless assumption will be kept throughout this paper, but we shall assume an infinitesimal anti-Hermitian part for $k$, having minus pure imaginary eigenvalues, if necessary; the latter assumption corresponds to the outward propagating wave condition at infinity. 
The matrices $s_{i}$ and $\rho_{i}$ are all Hermitian and especially

$$
\gamma_{i}^{*}=\gamma_{i}^{\tau}=-\gamma_{i}
$$

where $\gamma_{i}^{*}$ and $\gamma_{i}^{\tau}$ are the complex conjugate and transposed matrices of $\gamma_{i}$, respectively.

The matrices $s_{i}$ and $\rho_{i}$ satisfy the following relations which are often very convenient to use:

$$
s_{i} s_{j} s_{k}+s_{k} s_{j} s_{i}=\delta_{i j} s_{k}+\delta_{j k} s_{i}, \quad s_{i} s_{j}-s_{j} s_{i}=i^{-1} \epsilon_{i j k} s_{k},
$$

$$
s_{1}^{2}+s_{2}^{2}+s_{3}^{2}=2, \rho_{1}^{2}=\rho_{2}^{2}=\rho_{3}^{2}=1, \rho_{1} \rho_{2}=-\rho_{2} \rho_{1}=i \rho_{3}, \text { etc. }
$$

Here $\epsilon_{i j k}$ is the antisymmetrical tensor corresponding to $\epsilon_{123}=1$; its elements are +1 or -1 as the number of permutations necessary to change the order of subscripts $i, j, k$ into the order $1,2,3$ is even or odd. From (2.2a), it especially follows, for any two orthogonal vectors $m$ and $n$ in the ordinary three-dimensional space, that

$(n \gamma)^{3}=n^{2}(n \gamma),(n \gamma)(m \gamma)^{2}=\left\{m^{2}-(m \gamma)^{2}\right\}(n \gamma),(n \gamma)(m \gamma)(n \gamma)=0$,

$$
(n \gamma)^{2}(m \gamma)^{2}=(m \gamma)^{2}(n \gamma)^{2}=n^{2} m^{2}-(m \times n \cdot \gamma)^{2} .
$$

The traces of these matrices are frequently used in actual computations, and the following formulas hold:

$$
\operatorname{Tr}^{\prime}\left(s_{i}\right)=0, \operatorname{Tr}^{\prime}\left(s_{i} s_{j}\right)=2 \delta_{i j}, \operatorname{Tr}^{\prime}\left(s_{i} s_{j} s_{k}\right)=i^{-1} \epsilon_{i j k}, \operatorname{Tr}^{\prime}\left(s_{i} s_{j} s_{k} s_{l}\right)=\delta_{i j} \delta_{k l}+\delta_{j k} \delta_{i l}
$$

Here $\operatorname{Tr}^{\prime}$ stands for the trace in the subspace of $s_{i}$ 's matrices.

Besides the wave equation (2.1), it is convenient to introduce the adjoint wave function $\bar{\psi}$ defined by

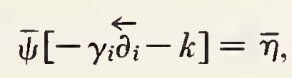

where $\bar{\eta}$ is an external source current for $\bar{\psi}$ which is independent of $\eta$, and $\overleftarrow{\partial_{i}}$ operates on the coordinates $x$ on the left side.

At infinity, the medium constant $k(x)$ is assumed to reduce adiabatically to a constant $k_{0}$ [Lippmann and Schwinger, 1950], while $\eta(x)=\bar{\eta}(x)$ should vanish there; this enables us to define the outward and inward propagating waves explicitly there. Also, the field at any point in space will be represented by a complete set of orthogonal modes of waves which can describe any field distribution on a plane perpendicular to the $x_{3}$-axis, passing through the point considered.

In free space or at infinity where $k=k_{0}$, the wave equations (2.1) and (2.3) become

$$
\left[(\gamma \partial)-k_{0}\right] \psi=\bar{\psi}\left[-(\stackrel{\leftarrow}{\partial})-k_{0}\right]=0
$$

which will have a complete set of solutions of plane waves as follows:

$$
\psi_{\lambda}(x)=e^{-i\left\{\lambda_{1} x_{1}+\lambda_{2} x_{2}+\lambda_{3} x_{3}\right\}} \xi_{\lambda}, \quad \bar{\psi}_{\lambda}(x)=e^{+i\left\{\lambda_{1} x_{1}+\lambda_{2} x_{2}+\lambda_{3} x_{3}\right\}} \bar{\xi}_{\lambda},
$$

where $\lambda_{1}$ and $\lambda_{2}$ are arbitrary real values and $\lambda_{3}$ 's are roots of

$$
\operatorname{Det}\left[(\lambda \gamma)-k_{0}\right]=0 \text {, }
$$

and $\xi_{\lambda}$ and $\bar{\xi}_{\lambda}$ are the solutions of

$$
\left[(\lambda \gamma)-k_{0}\right] \xi_{\lambda}=\bar{\xi}_{\lambda}\left[(\lambda \gamma)-k_{0}\right]=0 .
$$

\footnotetext{
${ }^{4}$ For example, in the case of an isotropic medium, $\lambda_{3}$ is given by $\pm \sqrt{(\omega / c)^{2} \epsilon \mu-\lambda_{1}^{2}-\lambda_{2}^{2}}$.
} 
There exists an orthogonal relation between the $\underline{\xi}_{\lambda}{ }^{\prime}$ s and $\bar{\xi}_{\lambda}$ s. In fact, if $\xi_{\lambda^{\prime}}$ and $\bar{\xi}_{\lambda^{\prime \prime}}$ are solutions of (2.7) for the same $\lambda_{1}$ and $\lambda_{2}$, it follows that

$$
\bar{\xi}_{\lambda^{\prime \prime}}\left[\lambda_{1} \gamma_{1}+\lambda_{2} \gamma_{2}-k_{0}\right] \xi_{\lambda^{\prime}}=-\lambda_{3}^{\prime \prime} \bar{\xi}_{\lambda^{\prime \prime}} \gamma_{3} \xi_{\lambda^{\prime}}=-\lambda_{3}^{\prime} \bar{\xi}_{\lambda^{\prime}} \gamma_{3} \xi_{\lambda^{\prime}}
$$

Hence, if $\lambda_{3}^{\prime \prime} \neq \lambda_{3}^{\prime}$,

$$
\bar{\xi}_{\lambda^{\prime \prime}} \gamma_{3} \xi_{\lambda^{\prime}}=0, \quad \lambda_{3}^{\prime \prime} \neq \lambda_{3}^{\prime},
$$

and, therefore, the following normalization is possible:

$$
\bar{\xi}_{\lambda^{\prime}} \gamma_{3} \xi_{\lambda^{\prime}}=\delta_{\lambda_{3} \lambda_{3}}^{\prime \prime \prime},
$$

where the $\lambda_{1}$ and $\lambda_{2}$-components of $\lambda^{\prime}$ and $\lambda^{\prime \prime}$ are to be the same, respectively.

In the following, the subscript $\lambda$ of $\xi_{\lambda}$ will stand for not only the $\lambda_{1}, \lambda_{2}$, and $\lambda_{3}$-components but also for a kind of polarization for the same $\lambda$, if necessary, so that the orthogonality similar to (2.10) holds between the $\xi_{\lambda}$ 's of different polarizations. Thus, from (2.5) and (2.10), there results the surface integral over the plane $x_{3}=0$

$$
\int_{x_{3}=0} \bar{\psi}_{\lambda^{\prime}} \gamma_{3} \psi_{\lambda^{\prime}} d \sigma_{3}=(2 \pi)^{2} \delta\left(\lambda_{1}^{\prime \prime}-\lambda_{1}^{\prime}\right) \delta\left(\lambda_{2}^{\prime \prime}-\lambda_{2}^{\prime}\right) \delta_{\lambda_{3^{\prime \prime} \lambda^{\prime}}}, \quad d \sigma_{3}=d x_{1} d x_{2} .
$$

Although the $\psi_{\lambda}$ 's thus defined constitute a continuous set of orthogonal functions, we may obtain a discrete set of orthogonal functions by restricting the integration domain of the surface integral on the left side of (2.11). Thus, if the whole system is assumed to be enclosed in a large square column with the cross section of $-L \leqslant x_{i} \leqslant+L(i=1,2)$, while $-\infty<x_{3}<+\infty$, the periodic function condition leads to the discrete set of values $\pi n / L(n=0, \pm 1, \pm 2, \ldots)$ for $\lambda_{1}$ and $\lambda_{2}$. The normalization condition (2.11) is then to be replaced by

$$
\int_{x_{3}=0} \bar{\psi}_{\lambda^{\prime}} \gamma_{3} \psi_{\lambda^{\prime}} d \sigma_{3}=\delta_{\lambda^{\prime \prime} \lambda^{\prime}} \equiv \delta_{\lambda_{1}^{\prime \prime} \lambda_{1}} \delta_{\lambda_{2}^{\prime \prime} \lambda_{2}^{\prime}} \delta_{\lambda_{3}^{\prime \prime} \lambda_{3}^{\prime}}
$$

For $L \rightarrow \infty$, the same results will be obtained as in the case of an unbounded space.

On the other hand, the complex conjugate functions $\psi_{\lambda}^{*}$ s satisfy

$$
\psi_{\lambda}^{*}\left[-(\overleftarrow{\gamma} \partial)-k_{0}\right]=0
$$

which is the same equation as for the $\bar{\psi}_{\lambda}$ 's in (2.4). Hence, it follows that $\bar{\psi}_{\lambda}$ can be expressed in terms of the $\psi_{\lambda}^{*}$ 's according to

$$
\bar{\psi}_{\lambda^{\prime}}=N_{\lambda^{\prime} \lambda^{\prime \prime}} \psi_{\lambda^{\prime \prime}}^{*}
$$

where the summation convention for quantities having the same subscripts is used here and henceforth. From the definitions of $\psi_{\lambda}$ and $\bar{\psi}_{\lambda}$ in (2.5), we infer that the matrix $N_{\lambda^{\prime} \lambda^{\prime \prime}}$ (with respect to the indices $\lambda^{\prime}$ and $\lambda^{\prime \prime}$ ) has nonvanishing matrix elements only for such $\lambda^{\prime}$ and $\lambda^{\prime \prime}$ having the same $\lambda_{1}$ - and $\lambda_{2^{-}}$-components.

Using (2.14) and (2.12), we find

$$
N_{\lambda^{\prime \prime} \lambda^{\prime \prime \prime}} \int_{x_{3}=0} \psi_{\lambda^{\prime \prime \prime \prime}}^{*} \gamma_{3} \psi_{\lambda^{\prime}} d \sigma_{3}=\delta_{\lambda^{\prime \prime} \lambda^{\prime}}
$$

so that the matrix $N$ is defined by the relation

$$
\int_{x_{3}=0} \psi_{\lambda^{\prime},}^{*} \gamma_{3} \psi_{\lambda^{\prime}} d \sigma_{3}=\left(N^{-1}\right)_{\lambda^{\prime \prime} \lambda^{\prime}}
$$


which is a Hermitian matrix, as is seen from the left side. For later convenience, we shall normalize the $\psi_{\lambda}$ 's such as to have

$$
|\operatorname{Det}(N)|=1 .
$$

From the orthogonality condition (2.15), we may expect the possibility to expand any $\psi$-function of the form $\gamma_{3}^{2} \psi$ in a linear combination of $\gamma_{3}^{2} \psi_{\lambda}$. Since $\gamma_{3}^{3}=\gamma_{3}$ according to $(2.2 \mathrm{~b})$, the eigenvalues of $\gamma_{3}$ are found to be either 0 or \pm 1 , and thus the components of $\psi_{\lambda^{\prime}}$ and $\bar{\psi} \lambda_{\lambda^{\prime \prime}}$ for which $\gamma_{3}$ has zero eigenvalue do not contribute to the normalization (2.15). Also, since $\gamma_{3}^{2}\left(\gamma_{3}^{2} \psi\right)=\left(\gamma_{3}^{2} \psi\right), \gamma_{3}^{2} \psi$ is the component of $\psi$ corresponding to the eigenvalue of $\gamma_{3}^{2}=1$, for which there is no component with the eigenvalue of $\gamma_{3}=0$. Thus, we may decompose an arbitrary $\psi(x)$ on any plane, say of $x_{3}=0$, into two parts,

with

$$
\psi(x)=\psi(\sigma(x))+\psi(n(x))
$$

$$
\gamma_{3}^{2} \psi(x)=\psi(\sigma(x)),\left(1-\gamma_{3}^{2}\right) \psi(x)=\psi(n(x))
$$

where $\psi(\sigma(x))$ represents the tangential components of the electromagnetic field, and $\psi(n(x))$ the normal components with respect to the surface $\sigma$ of $x_{3}=0$.

The component $\psi(n(x))$ is not independent of $\psi(\sigma(x))$, but can be derived from the latter with the aid of the wave equation (2.1) (appendix 1):

$\psi(n(x))=k_{33}^{-1}\left(1-\gamma_{3}^{2}\right)\left[\left\{\left(\gamma \partial_{T}\right)-k\right\} \psi(\sigma(x))-\eta(x)\right]$

$$
\bar{\psi}(n(x))=\left[\bar{\psi}(\sigma(x))\left\{-\left(\gamma \stackrel{\leftarrow}{\partial_{T}}\right)-k\right\}-\bar{\eta}(x)\right]\left(1-\gamma_{3}^{2}\right) k_{33}^{-1}
$$

where $n$ is the unit vector in the direction of the $x_{3}$-axis,

$$
k_{33}=\operatorname{Tr}^{\prime}\left[\left(1-\gamma_{3}^{2}\right) k\right], \quad \partial_{T}=\left(\partial_{1}, \partial_{2}, 0\right),
$$

and $T r^{\prime}$ is the trace of the referred matrix in the subspace of the $s_{i}$ 's matrices (see (2.2c)).

Now any $\psi(\sigma(x))$ and $\bar{\psi}(\sigma(x))$ may be expanded as ${ }^{5}$

$$
\psi(\sigma(x))=\psi_{\lambda^{\prime}}(\sigma(x)) N_{\lambda^{\prime} \lambda^{\prime \prime}} \psi_{\lambda^{\prime \prime}}[\sigma], \quad \bar{\psi}(\sigma(x))=\bar{\psi}_{\lambda^{\prime \prime}}[\sigma] N_{\lambda^{\prime \prime} \lambda^{\prime}} \psi_{\lambda^{\prime}}^{*}(\sigma(x)),
$$

where, in view of (2.16),

$$
\int_{x_{3}=0} \psi_{\lambda^{\prime \prime}}^{*}(\sigma(x)) \gamma_{3} \psi_{\lambda^{\prime}}(\sigma(x)) d \sigma_{3}=\left(N^{-1}\right) \lambda_{\lambda^{\prime \prime} \lambda^{\prime}}
$$

and thus

$$
\psi_{\lambda^{\prime}}[\sigma]=\int_{\sigma} \psi_{\lambda^{\prime}}^{*} \gamma_{3} \psi d \sigma_{3}, \quad \bar{\psi}_{\lambda^{\prime}}[\sigma]=\int_{\sigma} \bar{\psi} \gamma_{3} \psi_{\lambda^{\prime}} d \sigma_{3}
$$

and $\sigma$ stands for the surface $x_{3}=0$ in this case. Therefore, we also infer, from the completeness of the orthogonal functions, that

$$
\left\{\psi_{\lambda^{\prime}}(\sigma(x))\right\}_{\alpha} N_{\lambda^{\prime} \lambda^{\prime \prime}}\left\{\psi_{\lambda^{\prime \prime}}^{*}\left(\sigma\left(x^{\prime}\right)\right)\right\}_{\beta}=\left(\gamma_{3}\right)_{\alpha \beta} \delta\left(\sigma(x)-\sigma\left(x^{\prime}\right)\right),
$$

where the $\delta$-function on the right side is the two dimensional $\delta$-function on the plane $\sigma$.

${ }^{5}$ Generally, when $\eta(x) \neq 0$ and/or $k(x) \neq k_{0}, \psi(n(x)) \neq \psi_{\lambda^{\prime}}(n(x)) N_{\lambda^{\prime} \lambda^{\prime \prime}} \psi_{\lambda^{\prime \prime}}[\sigma]$. 
So far we have been considering the field on the special plane of $x_{3}=0$ and its representation by the complete set of plane wave solutions of (2.5), which, on the plane $x_{3}=0$, become

$$
\psi_{\lambda}(\sigma(x))=e^{-i\left\{\lambda_{1} x_{1}+\lambda_{2} x_{2}\right\}} \gamma_{3}^{2} \xi_{\lambda}, \quad \bar{\psi}_{\lambda}(\sigma(x))=e^{+i\left\{\lambda_{1} x_{1}+\lambda_{2} x_{2}\right\}} \bar{\xi}_{\lambda} \gamma_{3}^{2}
$$

Since we could do the same on other planes for which $x_{3} \neq 0$, we shall redefine $\psi_{\lambda}(\sigma(x))$ and $\bar{\psi}_{\lambda}(\sigma(x))$ by (2.20), which thus become independent of the coordinate $x_{3}$, in the following. Then the relations $(2.19 \mathrm{a}-\mathrm{d})$ are valid on any plane $\sigma$ perpendicular to the $x_{3}$-axis, and in view of (2.18) and (2.19), the whole field distribution on $\sigma$ can be obtained in terms of $\psi_{\lambda^{\prime}}[\sigma]$.

Besides the plane wave solutions (2.20), any other sets of wave functions, e.g., those based on the cylindrical coordinate system, might be used as well to represent the field on the plane $\sigma$. Therefore, in the following, we shall exclusively use the notation $\psi_{a}(\sigma(x))$ (having Latin subscripts), instead of $\psi_{\lambda}(\sigma(x))$, to mark a complete set of orthogonal functions for representing the field in $\sigma$. Correspondingly, $\psi_{\lambda}[\sigma]$ will be replaced by $\psi_{a}[\sigma]$, and the Greek subscripts will exclusively be used for representing the (six) components of the wave function.

Now $\psi_{a}\left[\sigma_{1}\right]$ and $\psi_{b}\left[\sigma_{2}\right]$ on different surfaces $\sigma_{1}$ and $\sigma_{2}$ are linearly connected as follows: let $\cup_{a}\left(x,\left[\sigma_{2}\right]\right)$ be the solution of the wave equation satisfying the boundary condition that $\gamma_{3}^{2} \cup_{a}\left(x,\left[\sigma_{2}\right]\right) \equiv \cup_{a}\left(\sigma(x),\left[\sigma_{2}\right]\right)$ tends to $\psi_{a}(\sigma(x))$ as $\sigma \rightarrow \sigma_{2}$. Thus,

$$
[(\gamma \partial)-k] \cup_{a}\left(x,\left[\sigma_{2}\right]\right)=0,\left.\cup_{a}\left(\sigma(x),\left[\sigma_{2}\right]\right)\right|_{\sigma \rightarrow \sigma_{2}}=\psi_{a}(\sigma(x)) .
$$

Here, according to $(2.19 \mathrm{a}-\mathrm{c})$,

$$
\cup_{a}\left(\sigma_{1}(x),\left[\sigma_{2}\right]\right)=\psi_{c}\left(\sigma_{1}(x)\right) N_{c b} \cup_{b a}\left[\sigma_{1}, \sigma_{2}\right],
$$

where, also in the following, $\sigma(x)$ is replaced by $\sigma_{1}(x)$ when the point $x$ is on a particular surface $\sigma_{1}$, and

$$
\cup_{b a}\left[\sigma_{1}, \sigma_{2}\right]=\int_{\sigma_{1}} \psi_{b}^{*} \gamma_{3} \cup_{a}\left(\sigma(x),\left[\sigma_{2}\right]\right) d \sigma_{3}
$$

The boundary condition in (2.21) corresponds to the condition

$$
\left.\cup_{b a}\left[\sigma_{1}, \sigma_{2}\right]\right|_{\sigma_{1} \rightarrow \sigma_{2}}=\left(N^{-1}\right)_{b a} .
$$

On the other hand, the complex conjugation of (2.21) and (2.22) yields

$$
\begin{aligned}
& \cup_{a}^{*}\left(x,\left[\sigma_{2}\right]\right)[-(\check{\gamma} \dot{\partial})-k]=0, \\
& \cup_{a}^{*}\left(\sigma_{1}(x),\left[\sigma_{2}\right]\right)=\cup_{a b}^{+}\left[\sigma_{2}, \sigma_{1}\right] N_{b c} \psi_{c}^{*}\left(\sigma_{1}(x)\right),
\end{aligned}
$$

where

$$
\cup_{a b}^{+}\left[\sigma_{2}, \sigma_{1}\right]=\cup_{b a}^{*}\left[\sigma_{1}, \sigma_{2}\right]
$$

Therefore, the matrix $\cup^{+}\left[\sigma_{2}, \sigma_{1}\right]$, thus defined, is the Hermitian conjugate of $\cup\left[\sigma_{1}, \sigma_{2}\right]$. Now, since

$$
\partial_{i}\left[\cup_{a}^{*}\left(x,\left[\sigma_{1}\right]\right) \gamma_{i} \cup_{b}\left(x,\left[\sigma_{2}\right]\right)\right]=0
$$

because of the wave equations in (2.21) and (2.25), it follows from the gaussian theory that

$$
\int_{\sigma} \cup_{a}^{*}\left(x,\left[\sigma_{1}\right]\right) \gamma_{3} \cup_{b}\left(x,\left[\sigma_{2}\right]\right) d \sigma_{3}=\cup_{a c}^{+}\left[\sigma_{1}, \sigma\right] N_{c d} \cup_{d b}\left[\sigma, \sigma_{2}\right]=\text { const },
$$


independent of the position of the surface $\sigma$. Therefore, setting $\sigma=\sigma_{2}$ and $\sigma=\sigma_{1}$ in (2.28) and using the boundary condition (2.24), we see that, since $N_{a b}^{+}=N_{a b}$ is Hermitian from (2.16),

$$
\cup_{a b}^{+}\left[\sigma_{1}, \sigma_{2}\right]=\cup_{a b}\left[\sigma_{1}, \sigma_{2}\right] .
$$

Thus $\cup\left[\sigma_{1}, \sigma_{2}\right]$ turns out to be the Hermitian conjugate of $\cup\left[\sigma_{2}, \sigma_{1}\right]$.

In the same way, we also see from (2.28) that, by replacing $\sigma_{2}$ by $\sigma_{1}$ and $\sigma$ by $\sigma_{2}$,

$$
\cup_{a c}\left[\sigma_{1}, \sigma_{2}\right] N_{c d} \cup_{d b}\left[\sigma_{2}, \sigma_{1}\right]=N^{-1} a b,
$$

which gives

$$
\cup_{G h b}^{-1}\left[\sigma_{1}, \sigma_{2}\right]=N_{a c} \bigcup_{c d}\left[\sigma_{2}, \sigma_{1}\right] N_{d b},
$$

or, symbolically,

$$
\cup^{-1}\left[\sigma_{1}, \sigma_{2}\right]=N \cup\left[\sigma_{2}, \sigma_{1}\right] N,
$$

where $U^{-1}\left[\sigma_{1}, \sigma_{2}\right]$ is the reciprocal matrix of $U\left[\sigma_{1}, \sigma_{2}\right]$ defined by $\cup^{-1}\left[\sigma_{1}, \sigma_{2}\right] \cup\left[\sigma_{1}, \sigma_{2}\right]=1$.

From the definition of $\cup\left[\sigma_{1}, \sigma_{2}\right]$, it is now evident that $\psi\left[\sigma_{1}\right]$ and $\psi\left[\sigma_{2}\right]$ are connected by the relation

$$
\psi_{a}\left[\sigma_{1}\right]=\cup_{a b}\left[\sigma_{1}, \sigma_{2}\right] N_{b c} \psi_{c}\left[\sigma_{2}\right], \quad \eta=0
$$

or, simply,

$$
\psi\left[\sigma_{1}\right]=\cup\left[\sigma_{1}, \sigma_{2}\right] N \psi\left[\sigma_{2}\right], \quad \eta=0,
$$

which is obtained, on using (2.19), from (2.22) with the boundary condition in (2.21).

We observe that the six-rowed matrix with respect to the Greek subscripts, $U\left(\sigma_{1}\left(x_{1}\right), \sigma_{2}\left(x_{2}\right)\right)$, defined by

$$
\begin{aligned}
\cup\left(\sigma_{1}\left(x_{1}\right), \sigma_{2}\left(x_{2}\right)\right) & =\cup_{a}\left(\sigma_{1}\left(x_{1}\right),\left[\sigma_{2}\right]\right) N_{a b} \psi_{b}^{*}\left(\sigma_{2}\left(x_{2}\right)\right) \\
& =\psi_{a}\left(\sigma_{1}\left(x_{1}\right)\right) N_{a b} \cup_{b c}\left[\sigma_{1}, \sigma_{2}\right] N_{c d} \psi_{d}^{*}\left(\sigma_{2}\left(x_{2}\right)\right)
\end{aligned}
$$

generally diverges and thus does not exist except for the case of $\sigma_{1}=\sigma_{2}$ where, because of the boundary condition (2.24) and $(2.19 \mathrm{~d})$,

$$
\cup\left(\sigma\left(x_{1}\right), \sigma\left(x_{2}\right)\right)=\gamma_{3} \delta\left(\sigma\left(x_{1}\right)-\sigma\left(x_{2}\right)\right), \quad \sigma_{1}=\sigma_{2}=\sigma .
$$

This situation is recognized as the special case of an isotropic homogeneous medium of $k=k_{0}$ by using the complete set of plane wave solutions $\psi_{\lambda}(x)$ of $(2.5)$ for $\psi_{a}(x)$; as observed in the footnote of (2.6), $\cup_{\lambda \lambda}\left[\sigma_{1}, \sigma_{2}\right]$ would then contain the factors $e^{-i \lambda_{3}\left(x_{3}-x_{3}{ }^{\prime}\right)}$ where $\lambda_{3} \rightarrow \pm i \infty$ as $\lambda_{1}, \lambda_{2} \rightarrow \pm \infty$. $^{6}$

In the general case of $\eta \neq 0$, the field at a point $x_{1}$ on $\sigma_{1}$ is determined by the boundary dis. tribution $\psi\left[\sigma_{2}\right]$ of the field at $\sigma_{2}$ and the external source $\eta$ between $\sigma_{1}$ and $\sigma_{2}$, and is given by (appendix 2).

$$
\begin{aligned}
\psi\left(x_{1}\right)=\sum_{a, b}\left[\cup_{a}\left(x_{1},\left[\sigma_{2}\right]\right) N_{a b} \psi_{b}\left[\sigma_{2}\right]\right. & \\
& \left.-i \int_{\sigma_{2}}^{\sigma_{1}} d x_{3}[\sigma] \cup_{a}\left(x_{1},[\sigma]\right) N_{a b} \int_{\sigma} \psi_{b}^{*}(x) \eta(x) d \sigma_{3}\right]-\left(1-\gamma_{3}^{2}\right) k_{33}\left(x_{1}\right)^{-1} \eta\left(x_{1}\right),
\end{aligned}
$$

${ }^{6}$ The general divergence of (2.33) involves the major difference from the case where $x_{3}$ is the time coordinate and $\psi$ is a wave function depending on the time explicitly; then $\lambda_{3}= \pm \sqrt{k_{0}^{2}+\lambda_{1}^{2}+\lambda_{2}^{3}} \rightarrow \pm \infty$ as $\lambda_{1}, \lambda_{2} \rightarrow \pm \infty$ so that $\cup\left(\sigma_{1}\left(x_{1}\right)\right.$, $\left.\sigma_{2}\left(x_{2}\right)\right)$ would exist generally. 
where

$$
\psi_{b}^{1} *(x)=\psi_{b}^{*}(\sigma(x))\left[1+\{-(\gamma \stackrel{\leftarrow}{\partial} \tau)-k\}\left(1-\gamma_{3}^{2}\right) k_{33}(x)^{-1}\right]
$$

is the complex conjugate field expected from the tangential components $\psi_{b}^{*}(\sigma(x))$ according to $(2.18 \mathrm{~b})$ in the medium of $k$ (but not of $k_{0}$ ). Now, all the integrals involved in (2.35) are convergent for any physically prescribed value of $\psi\left[\sigma_{2}\right]$, but the order of the integrations and the series sum. mation with respect to the Latin subscripts are not interchangeable in the series (2.35).

The equation (2.35) gives, using the matrix notation,

$$
\begin{aligned}
\psi\left[\sigma_{1}\right]=\cup\left[\sigma_{1}, \sigma_{2}\right] N \psi\left[\sigma_{2}\right]+\int_{\sigma_{2}}^{\sigma_{1}} d x_{3}[\sigma] \cup\left[\sigma_{1}, \sigma\right] N \eta[\sigma], \\
\eta_{a}[\sigma]=-i \int_{\sigma} \psi_{a}^{*}(\sigma(x))\left[1+\left\{\left(\vec{\partial}_{T}\right)-k\right\}\left(1-\gamma_{3}^{2}\right) k_{33}^{-1}\right] \eta(x) d \sigma_{3},
\end{aligned}
$$

which reduces to $(2.32 \mathrm{~b})$ in the case of $\eta=0$.

In the same way, we have the solution of (2.3) in terms of the boundary distribution $\bar{\psi}\left[\sigma_{2}\right]$ as follows:

$$
\begin{aligned}
\bar{\psi}\left[\sigma_{1}\right]=\bar{\psi}\left[\sigma_{2}\right] N \cup\left[\sigma_{2}, \sigma_{1}\right]+\int_{\sigma_{2}}^{\sigma_{2}} d x_{3}[\sigma] & \bar{\eta}[\sigma] N \cup\left[\sigma, \sigma_{1}\right], \\
& \bar{\eta}_{a}[\sigma]=i \int_{\sigma} \bar{\eta}(x)\left[1+k_{33}^{-1}\left(1-\gamma_{3}^{2}\right)\left\{-\left(\overleftarrow{\gamma}_{T}\right)-k\right\}\right] \psi_{a}(\sigma(x)) d \sigma_{3} .
\end{aligned}
$$

The complete set of wave functions $\psi_{a}(\sigma(x))$ can be divided into two groups, say $\psi_{a}^{ \pm}(\sigma(x))$, according to their directions of propagation. In the case of the plane wave functions $\psi_{\lambda}$ 's of (2.5), $\lambda_{3}$ is an analytic function of $\lambda_{1}$ and $\lambda_{2}$ in the whole range and tends to $\pm i \infty$ for $\left|\lambda_{1}\right|,\left|\lambda_{2}\right| \rightarrow \infty$, if an infinitesimal negative imaginary part of the medium constants are assumed, corresponding to an infinitesimal loss in the medium. Therefore, the set of wave functions $\psi_{\lambda}$ 's may be mathematically divided into the two groups whose $\lambda_{3}$ 's tend to $-i \infty$ and $+i \infty$, respectively, as $|\lambda|=\left(\lambda_{1}^{2}+\lambda_{2}^{2}\right)^{1 / 2} \rightarrow \infty$. As may be seen from the definitions in (2.5), the former group represents the waves propagating in the positive direction of the $x_{3}$-axis for the range of $|\lambda|$ where $\lambda_{3}$ assumes a real value, while the latter represents the waves propagating in the negative direction. Also, according to (2.5), the former group of solutions are not bounded in the domain $x_{3}<0$ for $|\lambda| \rightarrow \infty$, while the latter are not in the domain $x_{3}>0$. Thus, generally the two sets of functions $\psi_{\lambda}^{ \pm}(x)$, may be mathematically defined by those wave functions which are bounded in the domains $x_{3}>0$ and $x_{3}<0$, respectively, when $|\lambda| \rightarrow \infty$. This situation would be the same for the $\psi_{a}^{ \pm}$'s in the case of the most general orthogonal functions.

From (2.9), on noticing the different assignment of the phase terms for $\psi$ and $\bar{\psi}$, we deduce

$$
\int_{\sigma} \bar{\psi}_{a}^{ \pm} \gamma_{3} \psi_{b}^{ \pm} d \sigma_{3}=0
$$

where $\bar{\psi}_{a}^{ \pm}$'s are to be defined in the same way as for the $\psi_{a}^{ \pm}$'s.

\section{Introduction of a Probability Density Function for Waves}

As we have seen in the preceding section, the distribution of the wave function $\psi\left(x_{1}\right)$ on a surface, say $\sigma_{1}$, is completely described by its tangential components $\psi\left(\sigma_{1}\left(x_{1}\right)\right)$, which can be represented by $\psi_{a}\left[\sigma_{1}\right]$ 's (or simply $\psi\left[\sigma_{1}\right]$ ) as defined by (2.19). On the other hand, $\psi\left[\sigma_{1}\right]$ is completely determined if the boundary value $\psi\left[\sigma_{2}\right]$ is given on another surface $\sigma_{2}$, provided that the medium between $\sigma_{1}$ and $\sigma_{2}$ is prescribed. However, when the medium is fluctuating, $\psi\left[\sigma_{1}\right]$ would assume various values, and we could expect only a probability of having some value $\psi\left[\sigma_{1}\right]^{\prime}$ for a given boundary value $\psi\left[\sigma_{2}\right]^{\prime}$. We define the probability density function $\left\langle\psi\left[\sigma_{1}\right]^{\prime}, \sigma_{1} \mid \psi\left[\sigma_{2}\right]^{\prime}, \sigma_{2}\right\rangle$ in such a way that, for the given boundary value $\psi\left[\sigma_{2}\right]^{\prime}$ at $\sigma_{2}$, the probability that $\psi\left[\sigma_{1}\right]$ at $\sigma_{1}$ takes 
a value between $\psi\left[\sigma_{1}\right]^{\prime}$ and $\psi\left[\sigma_{1}\right]^{\prime}+d \psi\left[\sigma_{1}\right]^{\prime}$ is given by

$$
\left(d \psi\left[\sigma_{1}\right]^{\prime}\right)\left\langle\psi\left[\sigma_{1}\right]^{\prime}, \sigma_{1} \mid \psi\left[\sigma_{2}\right]^{\prime}, \sigma_{2}\right\rangle
$$

Here, $\left(d \psi\left[\sigma_{1}\right]^{\prime}\right)$ is defined by

$$
\left(d \psi\left[\sigma_{1}\right]^{\prime}\right)=\Pi_{a} d \psi_{a}\left[\sigma_{1}\right]_{R}^{\prime} d \psi_{a}\left[\sigma_{1}\right]_{I}^{\prime}
$$

where the subscripts $R$ and $I$ stand for the real and imaginary parts of the referring quantity, respectively.

Although $\psi\left[\sigma_{1}\right]^{\prime}$ and $\psi\left[\sigma_{2}\right]^{\prime}$ are quite independent of each other in most cases of a fluctuating medium, they are still bounded by the associated theory of reciprocity; from (2.37), we have, for a variation $d \psi\left[\sigma_{2}\right]^{\prime}$ of the boundary value on $\sigma_{2}$,

$$
d \psi\left[\sigma_{1}\right]^{\prime}=\cup\left[\sigma_{1}, \sigma_{2}\right] N d \psi\left[\sigma_{2}\right]^{\prime} .
$$

Therefore, according to appendix 3 ,

$$
\left(d \psi\left[\sigma_{1}\right]^{\prime}\right)=\left|\operatorname{Det}\left(\cup\left[\sigma_{1}, \sigma_{2}\right] N\right)\right|^{2}\left(d \psi\left[\sigma_{2}\right]^{\prime}\right)
$$

Here, in view of the normalization (2.17) and (2.30),

$$
\begin{aligned}
& |\operatorname{Det}(N)|=1 \text {, } \\
& \operatorname{Det}\left(\cup\left[\sigma_{1}, \sigma_{2}\right] N \cup\left[\sigma_{2}, \sigma_{1}\right]\right)=\operatorname{Det}\left(\cup\left[\sigma_{1}, \sigma_{2}\right]\right) \operatorname{Det}\left(\cup\left[\sigma_{2}, \sigma_{1}\right]\right) \\
& =\left|\operatorname{Det}\left(\cup\left[\sigma_{1}, \sigma_{2}\right]\right)\right|^{2},
\end{aligned}
$$

where (2.26) and (2.29) are taken into account.

Thus (3.4) reduces to

$$
\left(d \psi\left[\sigma_{1}\right]^{\prime}\right)=\left(d \psi\left[\sigma_{2}\right]^{\prime}\right),
$$

which is true, independent of the (lossless) medium between $\sigma_{1}$ and $\sigma_{2}$. By the substitution of (3.6) in (3.1), we infer that (3.1) also gives the probability that $\psi\left[\sigma_{2}\right]$ has a value between $\psi\left[\sigma_{2}\right]^{\prime}$ and $\psi\left[\sigma_{2}\right]^{\prime}+d \psi\left[\sigma_{2}\right]^{\prime}$ for the given value of $\psi\left[\sigma_{1}\right]^{\prime}$. Thus, according to the definition of (3.1),

$$
\left\langle\psi\left[\sigma_{1}\right]^{\prime}, \sigma_{1} \mid \psi\left[\sigma_{2}\right]^{\prime}, \sigma_{2}\right\rangle=\left\langle\psi\left[\sigma_{2}\right]^{\prime}, \sigma_{2} \mid \psi\left[\sigma_{1}\right]^{\prime}, \sigma_{1}\right\rangle .
$$

We shall see a more direct proof of (3.7) in section 5 .

\section{Lagrangian Variational Principle and Action Function of Waves}

As in quantum mechanics, the action function is fundamentally important also here in the statistical treatment of the waves. We define the Lagrangian density function $L$ by

$$
L=\bar{\psi}\left[\gamma_{i} \partial_{i}-k\right] \psi-\bar{\psi} \eta-\bar{\eta} \psi,
$$

and assume that the wave equations for $\psi$ and $\bar{\psi}$ are to be derived from the following variational principle for $\psi$ and $\bar{\psi}$, i.e.,

$$
\delta A=\delta \int_{\sigma_{2}}^{\sigma_{1}} L(d x)=0 .
$$

${ }^{7}$ Since the probability density function thus defined is a real function of both $\psi_{a}\left[\sigma_{1}\right]_{R}^{\prime}, \psi_{a}\left[\sigma_{1}\right]_{I}^{\prime}$, and $\psi_{a}\left[\sigma_{2}\right\}_{R}^{\prime}, \psi_{a}\left[\sigma_{2}\right]_{I}^{\prime}$, we should write it, strictly speaking, in the form

$$
\left\langle\psi\left[\sigma_{1}\right]^{\prime}, \psi^{*}\left[\sigma_{1}\right]^{\prime}, \sigma_{1} \mid \psi\left[\sigma_{2}\right]^{\prime}, \psi^{*}\left[\sigma_{2}\right]^{\prime}, \sigma_{2}\right\rangle
$$


Here, the space integration is to be performed over all the space between the surfaces $\sigma_{1}$ and $\sigma_{2}$, which are assumed as planes perpendicular to the $x_{3}$-axis; further, the variations $\delta \psi(x)$ and $\delta \bar{\psi}(x)$ are to vanish at the boundary surfaces.

On the other hand, adding the variations of $\eta(x), \bar{\eta}(x)$, and $k(x)$ for later convenience, we find with the aids of partial integration

$$
\begin{aligned}
\delta A=\int_{\sigma_{2}}^{\sigma_{1}}(d x)\left[\delta \bar{\psi}\left\{\left[\gamma_{i} \partial_{i}-k\right] \psi-\eta\right\}+\left\{\bar{\psi}\left[-\gamma_{i} \overleftarrow{\partial_{i}}-k\right]-\bar{\eta}\right\} \delta \psi\right] & \\
& +i\left[\int_{\sigma_{1}} \bar{\psi} \gamma_{3} \delta \psi d \sigma_{3}-\int_{\sigma_{2}} \bar{\psi} \gamma_{3} \delta \psi d \sigma_{3}\right]+\int_{\sigma_{2}}^{\sigma_{1}} \delta L(d x),
\end{aligned}
$$

where

$$
\delta L(x)=-\{\bar{\psi}(x) \delta \eta(x)+\delta \bar{\eta}(x) \psi(x)\}-\bar{\psi}(x) \delta k(x) \psi(x)
$$

According to the variational principle, the integrand of the first integral in (4.3) should vanish because it depends on the variations $\delta \psi$ and $\delta \bar{\psi}$ between $\sigma_{1}$ and $\sigma_{2}$, and thus the wave equations (2.1) and (2.3) are derived. The $\bar{\eta}(x)$ may be interpreted as a source current density for $\bar{\psi}(x)$. However, $\bar{\psi}$ has no physical meaning as yet and is introduced only for convenience.

Next we consider the variation $\delta A$ due to arbitrary displacements $\delta x_{j}(x)$ of the whole system, keeping the values of $\psi$ and $\bar{\psi}$ at every point, so that $\psi(x-\delta x)$ at the point $x-\delta x$ becomes the new wave function $\psi(x)$ at the point $x$ after the displacement. Hence, the corresponding variation $\delta \psi$ will be given by $\delta \psi=-\left(\partial / \partial x_{j}\right) \psi \delta x_{j}$, which are to be substituted in (4.3) for the variations of the wave function at the boundary surfaces.

Now the total variation $\delta_{x} A$ due to the displacement in space is obtained by adding the contribution due to the change of the integration domain: thus, assuming uniform and independent displacements $\delta x_{i}\left[\sigma_{1}\right]$ and $\left.\delta x_{i} \mid \sigma_{2}\right]$ on $\sigma_{1}$ and $\sigma_{2}$, respectively, we obtain

where

$$
\delta_{x} A=P_{j}\left[\sigma_{1}\right] \delta x_{j}\left[\sigma_{1}\right]-P_{j}\left[\sigma_{2}\right] \delta x_{j}\left[\sigma_{2}\right]
$$

$$
\begin{aligned}
P_{j}[\sigma] & =\int_{\sigma} T_{3 j} d \sigma_{3}, \\
T_{i j} & =-\bar{\psi} \gamma_{i} \partial_{j} \psi+L \delta_{i j} .
\end{aligned}
$$

With the aid of the wave equation (2.1) and the relation (2.18), all the $T_{i j}$ 's can be expressed in terms of the tangential components $\psi(\sigma(x)), \bar{\psi}(\sigma(x))$ and the tangential derivatives of $\psi(\sigma(x))$; for instance, in view of the identity $\gamma_{i} \partial_{i}-\gamma_{3} \partial_{3}=\gamma_{T} \partial_{T}$, we obtain from (4.6)

$$
\begin{aligned}
& T_{33}=\bar{\psi}\left[\left(\gamma \partial_{T}\right)-k\right] \psi-\bar{\psi} \eta-\bar{\eta} \psi \\
&=\bar{\psi}(\sigma)\left[\left\{\left(\gamma \partial_{T}\right)-k\right\} k_{33}^{-1}\left(1-\gamma_{3}^{2}\right)\left\{\left(\gamma \partial_{T}\right)-k\right\}-k\right] \psi(\sigma)-\bar{\eta}\left[1+k_{33}^{-1}\left(1-\gamma_{3}^{2}\right)\left\{\left(\gamma \partial_{T}\right)-k\right\}\right] \psi(\sigma) \\
& \quad-\bar{\psi}(\sigma)\left[1+\left\{\left(\gamma \partial_{T}\right)-k\right\}\left(1-\gamma_{3}^{2}\right) k_{33}^{-1}\right] \eta+\bar{\eta} k_{33}^{-1}\left(1-\gamma_{3}^{2}\right) \eta
\end{aligned}
$$

On the other hand, using the wave equations (2.1) and (2.3), $P_{3}[\sigma]$ is given in a simple form as

$$
\begin{aligned}
P_{3}[\sigma] & =\int_{\sigma}\left[\bar{\psi} \gamma_{3}^{2}\left\{\left(\left(\gamma \partial_{T}\right)-k\right) \psi-\eta\right\}-\bar{\eta} \psi\right] d \sigma_{3} \\
& =\int_{\sigma}\left[\left\{\bar{\psi}\left(-\left(\overleftarrow{\gamma}_{T}\right)-k\right)-\bar{\eta}\right\} \gamma_{3}^{2} \psi-\bar{\psi} \eta\right] d \sigma_{3}
\end{aligned}
$$

with the supplementary conditions 


$$
\begin{gathered}
\left(1-\gamma_{3}^{2}\right)\left\{\left(\left(\gamma \partial_{T}\right)-k\right) \psi-\eta\right\}=0 \\
\left\{\bar{\psi}\left(-\left(\overleftarrow{\gamma}_{\partial_{T}}\right)-k\right)-\bar{\eta}\right\}\left(1-\gamma_{3}^{2}\right)=0
\end{gathered}
$$

which define $\psi(n(x))$ and $\bar{\psi}(n(x))$ in terms of $\psi(\sigma(x)), \partial_{T} \psi(\sigma(x))$ and $\bar{\psi}(\sigma(x)), \partial_{T} \bar{\psi}(\sigma(x))$, respectively.

Thus, the total variation $\delta A$, amounting to the sum of (4.3) and (4.5), becomes

$$
\begin{aligned}
\delta A \equiv \delta A\left(\psi\left[\sigma_{1}\right], \sigma_{1} \mid \psi\left[\sigma_{2}\right], \sigma_{2}\right)=i \int_{\sigma_{1}} \bar{\psi}(\sigma) \gamma_{3} \delta \psi(\sigma) d \sigma_{3}+P_{j}\left[\sigma_{1}\right] \delta x_{j}\left[\sigma_{1}\right] \\
\\
\quad-i \int_{\sigma_{2}} \bar{\psi}(\sigma) \gamma_{3} \delta \psi(\sigma) d \sigma_{3}-P_{j}\left[\sigma_{2}\right] \delta x_{j}\left[\sigma_{2}\right]+\int_{\sigma_{2}}^{\sigma_{1}} \delta L(d x),
\end{aligned}
$$

which formerly gives the differential equation to be solved in order to obtain $A$ itself.

Now we conveniently introduce a surface functional derivative defined in such a way that we have from (4.8)

$$
\begin{aligned}
\delta A / \delta \psi_{\alpha}\left(\sigma_{1}(x)\right) & =i\left\{\bar{\psi}\left(\sigma_{1}(x)\right) \gamma_{3}\right\}_{\alpha} \\
-\delta A / \delta \psi_{\alpha}\left(\sigma_{2}(x)\right) & =i\left\{\bar{\psi}\left(\sigma_{2}(x)\right) \gamma_{3}\right\}_{\alpha},
\end{aligned}
$$

where $x$ is on the referred surface $\sigma_{1}$ or $\sigma_{2}$. Thus, from the completeness of the differential equation (4.8), i.e., $\left(\partial / \partial x_{3}[\sigma]\right)\left(\delta / \delta \psi_{\alpha}(\sigma(x))\right) A=\left(\delta / \delta \psi_{\alpha}(\sigma(x))\right)\left(\partial / \partial x_{3}[\sigma]\right) A$, we have

$$
\partial_{j}\left(\bar{\psi}(x) \gamma_{3}\right)_{\alpha}=\left(\delta / \delta \psi_{\alpha}(\sigma(x))\right) P_{j}[\sigma], \quad j=1,2,3 .
$$

In the same way, if we introduce $A^{\prime}$ defined by

$$
\begin{aligned}
& \qquad A^{\prime}=A\left(\psi\left[\sigma_{1}\right], \sigma_{1} \mid \psi\left[\sigma_{2}\right], \sigma_{2}\right)-i\left\{\int_{\sigma_{1}}-\int_{\sigma_{2}}\right\} \bar{\psi} \gamma_{3} \psi d \sigma_{3}, \\
& \text { we find, using (4.8), } \\
& \qquad \delta A^{\prime}=-i\left\{\int_{\sigma_{1}}-\int_{\sigma_{2}}\right\} \delta \bar{\psi} \gamma_{3} \psi d \sigma_{3}+P_{j}\left[\sigma_{1}\right] \delta x_{j}\left[\sigma_{1}\right]-P_{j}\left[\sigma_{2}\right] \delta x_{j}\left[\sigma_{2}\right]+\int_{\sigma_{2}}^{\sigma_{1}} \delta L(d x),
\end{aligned}
$$

which gives the equation corresponding to (4.10):

$$
-\partial_{j}\left(\gamma_{3} \psi(x)\right)_{\alpha}=\left(\delta / \delta \bar{\psi}_{\alpha}(\sigma(x))\right) P_{j}[\sigma]
$$

In the special case of $j=3,(4.12)$ together with (4.6) and (4.7) gives the wave equation in terms of $\psi(\sigma(x))$. Evidently (4.10) and (4.12) correspond to the Hamiltonian canonical equations in dynamics.

The explicit solution of (4.8) is obtained as follows: for arbitrary variations of $\psi\left(\sigma_{1}(x)\right), \psi\left(\sigma_{2}(x)\right)$ and $\eta(x)$,

$$
\delta A\left(\psi\left\lfloor\sigma_{1}\right], \sigma_{1} \mid \psi\left[\sigma_{2}\right], \sigma_{2}\right)=i\left\{\int_{\sigma_{1}}-\int_{\sigma_{2}}\right\} \bar{\psi} \gamma_{3} \delta \psi d \sigma_{3}-\int_{\sigma_{2}}^{\sigma_{1}} \bar{\psi} \delta \eta(d x)
$$

which gives, since $\bar{\psi}[\sigma]$ is given by (2.38) and thus the $\bar{\psi}$ is perfectly independent of $\psi$ and $\eta$,

$$
A\left(\psi\left[\sigma_{1}\right], \sigma_{1} \mid \psi\left[\sigma_{2}\right], \sigma_{2}\right)=i\left\{\int_{\sigma_{1}}-\int_{\sigma_{2}}\right\} \bar{\psi} \gamma_{3} \psi d \sigma_{3}-\int_{\sigma_{2}}^{\sigma_{1}} \bar{\psi} \eta(d x),
$$

where the integration constants are chosen such that $A$ vanishes for $\psi\left[\sigma_{1}\right]=\psi\left[\sigma_{2}\right]=\eta=0$.

The action $A$, given by (4.13), can be expressed in terms of $\psi\left[\sigma_{1}\right], \psi\left[\sigma_{2}\right], \eta[\sigma]$, and the corresponding quantities for $\bar{\psi}$ and $\bar{\eta}$ which are defined by (2.19), (2.37), and (2.38), and thus 
$A\left(\psi\left[\sigma_{1}\right], \sigma_{1} \mid \psi\left[\sigma_{2}\right], \sigma_{2}\right)=i\left\{\bar{\psi}\left[\sigma_{1}\right] N \psi\left[\sigma_{1}\right]-\bar{\psi}\left[\sigma_{2}\right] N \psi\left[\sigma_{2}\right]\right\}$

$$
-i \int_{\sigma_{2}}^{\sigma_{1}} \bar{\psi}[\sigma] N \eta[\sigma] d x_{3}[\sigma]+\int_{\sigma_{2}}^{\sigma_{1}} \bar{\eta}(x)\left(1-\gamma_{3}^{2}\right) k_{33}(x)^{-1} \eta(x)(d x)
$$

Here, although $\psi\left[\sigma_{1}\right]$ and $\psi\left[\sigma_{2}\right]$ are independent variables of $A, \bar{\psi}\left[\sigma_{1}\right]$ and $\bar{\psi}\left[\sigma_{2}\right]$ are not independent but are to be understood as related by (2.38); this is required from the "equation of motion" (4.10) for $j=3$ which is equivalent to the wave equation for $\bar{\psi}$.

In the special case of $\bar{\eta}=0,(4.14)$ takes the following form, when using (2.38) for $\bar{\psi}\left[\sigma_{1}\right]$ with the boundary value $\bar{\psi}\left[\sigma_{2}\right]^{\prime}$ and replacing $\psi\left[\sigma_{1}\right]$ by $\psi\left[\sigma_{1}\right]^{\prime}$ and $\psi\left[\sigma_{2}\right]$ by $\psi\left[\sigma_{2}\right]^{\prime}$,

$$
\begin{aligned}
A\left(\psi\left[\sigma_{1}\right]^{\prime}, \sigma_{1} \mid \psi\left[\sigma_{2}\right]^{\prime}, \sigma_{2}\right) & =i \bar{\psi}\left[\sigma_{2}\right]^{\prime} N\left\{\psi\left[\sigma_{2}\right]-\psi\left[\sigma_{2}\right]^{\prime}\right\} \\
& =i \bar{\psi}\left[\sigma_{1}\right] N\left\{\psi\left[\sigma_{1}\right]^{\prime}-\psi\left[\sigma_{1}\right]\right\} .
\end{aligned}
$$

Here, $\psi\left[\sigma_{1}\right], \psi\left[\sigma_{2}\right]$, and $\bar{\psi}\left[\sigma_{1}\right]$, having no primes, are the values determined from the boundary values $\psi\left[\sigma_{2}\right]^{\prime}, \psi\left[\sigma_{1}\right]^{\prime}$, and $\bar{\psi}\left[\sigma_{2}\right]^{\prime}$, respectively; according to (2.37) and (2.38),

$$
\begin{aligned}
& \psi\left[\sigma_{2}\right]=\cup\left[\sigma_{2}, \sigma_{1}\right] N \psi\left[\sigma_{1}\right]^{\prime}+\int_{\sigma_{1}}^{\sigma_{2}} \cup\left[\sigma_{2}, \sigma\right] N \eta[\sigma] d x_{3}[\sigma], \\
& \bar{\psi}\left[\sigma_{1}\right]=\bar{\psi}\left[\sigma_{2}\right]^{\prime} N \cup\left[\sigma_{2}, \sigma_{1}\right], \text { etc. }
\end{aligned}
$$

The proof of (4.15) is given in appendix 4.

\section{Equation Formulation of the Probability Density Function of Waves}

We first assume a given boundary value $\psi\left[\sigma_{1}\right]^{\prime}$ at $\sigma_{1}$ and ask the probability that $\psi\left[\sigma_{2}\right]$ at $\sigma_{2}$ will have a value in the range between $\psi\left[\sigma_{2}\right]^{\prime}$ and $\psi\left[\sigma_{2}\right]^{\prime}+d \psi\left[\sigma_{2}\right]^{\prime}$. Then, according to the definition (3.1) of the probability density function, it follows that

$$
\left\langle\psi\left[\sigma_{2}\right]^{\prime}, \sigma_{2} \mid \psi\left[\sigma_{1}\right]^{\prime}, \sigma_{1}\right\rangle=\operatorname{Av}\left[\delta\left(\psi\left[\sigma_{2}\right]^{\prime}-\psi\left[\sigma_{2}\right]\right)\right] .
$$

Here, $\psi\left[\sigma_{2}\right]$ is defined by (4.16) and is thus the value at $\sigma_{2}$ determined by the boundary value $\psi\left[\sigma_{1}\right]^{\prime}$; Av stands for the average value of referred variable over all the possible values of the medium constant $k$, and the complex $\delta$-function is defined by

$$
\delta\left(\psi[\sigma]^{\prime}-\psi[\sigma]^{\prime \prime}\right)=\Pi_{a} \delta\left(\psi_{a}[\sigma]_{R}^{\prime}-\psi_{a}[\sigma]_{R}^{\prime \prime}\right) \delta\left(\psi_{a}[\sigma]_{I}^{\prime}-\psi_{a}[\sigma]_{I}^{\prime \prime}\right)
$$

Indeed, if the medium does not fluctuate, the probability density is to be the $\delta$-function of (5.1).

By the use of the integral representation

$$
\delta(x)=\frac{1}{2 \pi} \int_{-\infty}^{\infty} e^{i \lambda x} d \lambda,
$$

and also the first expression of (4.15) for the action function $A$, we find the following fundamental relation between the action and the probability density function:

$$
\left\langle\psi\left[\sigma_{2}\right]^{\prime}, \sigma_{2} \mid \psi\left[\sigma_{1}\right]^{\prime}, \sigma_{1}\right\rangle=\operatorname{Av}\left[\int_{-\infty}^{\infty}\left(d \bar{\psi}\left[\sigma_{2}\right]^{\prime} N / 2 \pi\right) \exp \left[i \operatorname{Im}\left[A\left(\psi\left[\sigma_{1}\right]^{\prime}, \sigma_{1} \mid \psi\left[\sigma_{2}\right]^{\prime}, \sigma_{2}\right)\right]\right]\right] .
$$

Here, in view of (4.16) with (A.3.10) and (2.17),

$$
\left(d \bar{\psi}\left[\sigma_{2}\right]^{\prime} N\right)=\left(d \bar{\psi}\left[\sigma_{2}\right]^{\prime}\right)=\left(d \bar{\psi}\left[\sigma_{1}\right]\right)=\left(d \bar{\psi}\left[\sigma_{1}\right] N\right) .
$$


Therefore, using the second expression of (4.15) for $A$, it follows that (5.4) can be also expressed in the form

$$
\left\langle\psi\left[\sigma_{2}\right]^{\prime}, \sigma_{2} \mid \psi\left[\sigma_{1}\right]^{\prime}, \sigma_{1}\right\rangle=\operatorname{Av}\left[\delta\left(\psi\left[\sigma_{1}\right]^{\prime}-\psi\left[\sigma_{1}\right]\right)\right],
$$

whose right side is equal to $\left\langle\psi\left[\sigma_{1}\right]^{\prime}, \sigma_{1} \mid \psi\left[\sigma_{2}\right]^{\prime}, \sigma_{2}\right\rangle$ according to the definition (5.1). Thus, we have a direct proof of (3.7).

Now (5.4) can be expressed in the form

$$
\left\langle\psi\left[\sigma_{1}\right]^{\prime}, \sigma_{1} \mid \psi\left[\sigma_{2}\right]^{\prime}, \sigma_{2}\right\rangle=\operatorname{Av}\left[\int_{-\infty}^{\infty}\left(d \bar{\psi}\left[\sigma_{2}\right]^{\prime} / 2 \pi\right) \exp \left[\frac{1}{2}\left(A-A^{*}\right)\right]\right],
$$

where

$$
A=A\left(\psi\left[\sigma_{1}\right]^{\prime}, \sigma_{1} \mid \psi\left[\sigma_{2}\right]^{\prime}, \sigma_{2}\right),
$$

and $A^{*}$ is the complex conjugate of $A$.

Although (5.7) gives the probability density function only when $\bar{\eta}=0$, we shall keep $\bar{\eta} \neq 0$ in the following for mathematical convenience, with the understanding that $\bar{\eta}$ is to vanish in the final result.

\subsection{Matrix Representation of Fluctuating Medium and Representation of the Expec- tation Value as an Inner Product of Hilbert Vectors}

For a further development, we need assumptions concerning the distribution function of the medium constant $k$ in the wave equations (2.1) and (2.3). The $k$ is here assumed to be fluctuating around a mean value $k_{0}$ and thus will generally take the form (compare with (I.4.1) and (I.4.6a))

$$
k(x)=k_{0}+\gamma \phi(x),
$$

where both $k_{0}$ and $\gamma$ are constant Hermitian matrices (having the same physical dimension) operating on the $\psi$-vector, and $\phi(x)$ is a scalar real function. The $\gamma$ may be defined such as to have

$$
\operatorname{Av}[\phi(x)]=0, \quad \operatorname{Av}\left[\phi(x)^{2}\right]=1 .
$$

Here the medium fluctuation is assumed to be homogeneous over all the space except at infinity where the fluctuation is to vanish adiabatically.

We now introduce the distribution function $\left\langle\phi \mid \phi[\Sigma]^{\prime}\right\rangle$ which is defined so that the probability that $\phi[\Sigma]$, i.e., the distribution of $\phi$ over all the points of the space $\Sigma$ have some values between $\phi[\Sigma]^{\prime}$ and $\phi[\Sigma]^{\prime}+d \phi[\Sigma]^{\prime}$, is given by $\left\langle\phi \mid \phi[\Sigma]^{\prime}\right\rangle d \phi[\Sigma]^{\prime}$, the space $\Sigma$ being the whole space here (appendix 7).

The Fourier representation of $\langle\phi| \phi[\Sigma]$ ' $\rangle$ is usually called "characteristic function" and is very convenient in most cases. It is defined by the relations

$$
\begin{gathered}
\left\langle\phi \mid \phi[\Sigma]^{\prime}\right\rangle=\int_{-\infty}^{\infty}\left\langle\phi \mid \mu[\Sigma]^{\prime}\right\rangle d \mu[\Sigma]^{\prime}\left\langle\mu[\Sigma]^{\prime} \mid \phi[\Sigma]^{\prime}\right\rangle, \\
\left\langle\phi \mid \mu[\Sigma]^{\prime}\right\rangle=\int_{-\infty}^{\infty}\left\langle\phi \mid \phi[\Sigma]^{\prime}\right\rangle d \phi[\Sigma]^{\prime}\left\langle\phi[\Sigma]^{\prime} \mid \mu[\Sigma]^{\prime}\right\rangle,
\end{gathered}
$$

where [ compare (A.7.20)]

${ }^{8}$ The definition of $d \mu[\Sigma]^{\prime}$ and $d \phi[\Sigma]^{\prime}$ should be given properly (appendix 7). Also, the upper and lower limits of integrations with respect to $\phi[\Sigma]^{\prime}$ and $\mu[\Sigma]^{\prime}$ are always to be $\pm \infty$ and will not be assigned explicitly in the following. 


$$
\begin{aligned}
& \left\langle\mu[\Sigma]^{\prime} \mid \phi[\Sigma]^{\prime}\right\rangle=\exp \left[i \int_{\Sigma} \mu(x)^{\prime} \phi(x)^{\prime}(d x)\right], \\
& \left\langle\phi[\Sigma]^{\prime} \mid \mu[\Sigma]^{\prime}\right\rangle=\left\langle\mu[\Sigma]^{\prime} \mid \phi[\Sigma]^{\prime}\right\rangle^{*}=\exp \left[-i \int_{\Sigma} \mu(x)^{\prime} \phi(x)^{\prime}(d x)\right], \\
& \int\left\langle\phi[\Sigma]^{\prime} \mid \mu[\Sigma]^{\prime}\right\rangle d \mu[\Sigma]^{\prime}\left\langle\mu[\Sigma]^{\prime} \mid \phi[\Sigma]^{\prime \prime}\right\rangle=\delta\left(\phi[\Sigma]^{\prime}-\phi[\Sigma]^{\prime \prime}\right), \\
& \int\left\langle\mu[\Sigma]^{\prime} \mid \phi[\Sigma]^{\prime}\right\rangle d \phi[\Sigma]^{\prime}\left\langle\phi[\Sigma]^{\prime} \mid \mu[\Sigma]^{\prime \prime}\right\rangle=\delta\left(\mu[\Sigma]^{\prime}-\mu[\Sigma]^{\prime \prime}\right),
\end{aligned}
$$

and, from the normalization condition of the distribution function,

$$
\left.\left\langle\phi \mid \mu[\Sigma]^{\prime}\right\rangle\right|_{\mu^{\prime}=0}=\int\left\langle\phi \mid \phi[\Sigma]^{\prime}\right\rangle d \phi[\Sigma]^{\prime}=1 .
$$

When the fluctuation follows the gaussian multivariate distribution with the mean value zero as in (5.9), $\left\langle\phi \mid \mu[\Sigma]^{\prime}\right\rangle$ is assumed to take the form

$$
\left\langle\phi \mid \mu[\Sigma]^{\prime}\right\rangle=\exp \left[-\frac{1}{2} \iint_{\Sigma} \mu(x)^{\prime} D(x-y) \mu(y)^{\prime}(d x)(d y)\right] .
$$

Here the function $D(x-y)$ is easily identified as the expectation value of the product $\phi(x) \phi(y)$; i.e.,

$$
\operatorname{Av}[\phi(x) \phi(y)]=\int\left\langle\phi \mid \phi[\Sigma]^{\prime}\right\rangle d \phi[\Sigma]^{\prime} \phi(x)^{\prime} \phi(y)^{\prime}=D(x-y),
$$

which is derived by the use of $(5.10),(5.12),(5.13)$, and the partial integration together with

$$
\left(\delta / \delta \mu(x)^{\prime}\right)\left\langle\phi \mid \mu[\Sigma]^{\prime}\right\rangle=-\int_{\Sigma} D(x-y) \mu(y)^{\prime}(d y)\left\langle\phi \mid \mu[\Sigma]^{\prime}\right\rangle,
$$

where the left side is the functional derivative as ordinarily defined.

There exists an operational method for getting the average value Av $[Q]$ of any functional $Q(\phi[\Sigma])$ over all possible distributions of the medium. Thus, using the Fourier transformation (5.10) with (5.11),

$$
\begin{aligned}
\operatorname{Av}[Q(\phi)] & =\int\left\langle\phi \mid \phi[\Sigma]^{\prime}\right\rangle Q\left(\phi^{\prime}\right) d \phi[\Sigma]^{\prime} \\
& =\iint\left\langle\phi \mid \mu[\Sigma]^{\prime}\right\rangle d \mu[\Sigma]^{\prime}\left\langle\mu[\Sigma]^{\prime}|Q(\phi)| \mu[\Sigma]^{\prime \prime}\right\rangle d \mu[\Sigma]^{\prime \prime} \int\left\langle\mu[\Sigma]^{\prime \prime} \mid \phi[\Sigma]^{\prime}\right\rangle d \phi[\Sigma]^{\prime},
\end{aligned}
$$

where

$$
\left.\left\langle\mu[\Sigma]^{\prime}|Q(\phi)| \mu[\Sigma]^{\prime \prime}\right\rangle=\int\langle\mu \dot{\Sigma} \Sigma]^{\prime}\left|\phi[\Sigma]^{\prime}\right\rangle Q\left(\phi^{\prime}\right)\left\langle\phi[\Sigma]^{\prime} \mid \mu[\Sigma]^{\prime \prime}\right\rangle d \phi \dot{[} \Sigma\right]^{\prime}
$$

Hence, when $Q=\phi(x)$ or $\phi(x)^{n}$, we have on reference to (5.11b)

$$
\begin{array}{r}
\left\langle\mu[\Sigma] \cdot|\phi(x)| \mu[\Sigma]^{\prime \prime}\right\rangle=\left(-i \delta / \delta \mu(x)^{\prime}\right) \delta\left(\mu[\Sigma]^{\prime}-\mu[\Sigma]^{\prime \prime}\right), \\
\left\langle\mu[\Sigma]^{\prime}\left|\phi(x)_{n}\right| \mu[\Sigma]^{\prime \prime}\right\rangle=\left(-i \delta / \delta \mu(x)^{\prime}\right)^{n} \delta\left(\mu[\Sigma]^{\prime}-\mu[\Sigma]^{\prime \prime}\right) .
\end{array}
$$

Here we conveniently introduce the "matrices" $\phi(x)$ and $\mu(x)$ whose matrix elements are defined by $\left\langle\mu[\Sigma]^{\prime}|\phi(x)| \mu[\Sigma]^{\prime \prime}\right\rangle$ in (5.18) and

$$
\left\langle\mu[\Sigma]^{\prime}|\mu(x)| \mu[\Sigma]^{\prime \prime}\right\rangle=\mu(x)^{\prime} \delta\left(\mu[\Sigma]^{\prime}-\mu[\Sigma]^{\prime \prime}\right),
$$

the latter being a diagonal matrix. Then, using the ordinary matrix multiplication rule

$$
\left\langle\mu^{\prime}|A B| \mu^{\prime \prime}\right\rangle=\int\left\langle\mu^{\prime}|A| \mu^{\prime \prime \prime}\right\rangle d \mu^{\prime \prime \prime}\left\langle\mu^{\prime \prime \prime}|B| \mu^{\prime \prime}\right\rangle,
$$


$\left\langle\mu[\Sigma]^{\prime}\left|\phi(x)^{n}\right| \mu[\Sigma]^{\prime \prime}\right\rangle$ in (5.18) can be obtained as the matrix element of the matrix $\phi(x)^{n}$. More generally, if $Q(\phi)$ is a functional expandible with respect to $\phi,(5.17)$ can be regarded as the matrix element of the corresponding matrix $Q(\phi)$.

On the other hand, using the representations of $\phi(x)$ and $\mu(x)$ in (5.18) and (5.19), we observe that their commutation relations are

$$
\begin{gathered}
{\left[\phi(x), \mu\left(x^{\prime}\right)\right]=-i \delta\left(x-x^{\prime}\right),} \\
{\left[\phi(x), \phi\left(x^{\prime}\right)\right]=\left[\mu(x), \mu\left(x^{\prime}\right)\right]=0}
\end{gathered}
$$

with the ordinary bracket convention for commutator

$$
[A, B] \equiv A B-B A
$$

Now, on the right side of (5.16), $\left\langle\phi \mid \mu[\Sigma]^{\prime}\right\rangle$ is naturally interpreted as the representation of a left-hand Hilbert vector $\langle\phi|$ on which the matrix $Q(\phi)$ operates from the right side, and the term

$$
\int\left\langle\mu[\Sigma]^{\prime \prime} \mid \phi[\Sigma]^{\prime}\right\rangle d \phi[\Sigma]^{\prime}=\delta\left(\mu[\Sigma]^{\prime \prime}\right) \equiv\left\langle\mu[\Sigma]^{\prime \prime} \mid 0_{\mu}\right\rangle
$$

as the representation of a right-hand Hilbert vector $\left|0_{\mu}\right\rangle$ on which $Q(\phi)$ operates from the left side. Thus (5.16) may be symbolically expressed as

$$
\operatorname{Av}[Q(\phi)]=\left\langle\phi|Q(\phi)| 0_{\mu}\right\rangle
$$

with the normalization

$$
\left\langle\phi \mid 0_{\mu}\right\rangle=\int\left\langle\phi \mid \mu[\Sigma]^{\prime}\right\rangle d \mu[\Sigma]^{\prime}\left\langle\mu[\Sigma]^{\prime} \mid 0_{\mu}\right\rangle=\left.\left\langle\phi \mid \mu[\Sigma]^{\prime}\right\rangle\right|_{\mu^{\prime}=0}=1,
$$

which follows from (5.12). Here, the Hilbert vectors $\langle\phi|$ and $\left|0_{\mu}\right\rangle$ are specialized by (5.15) and (5.23), respectively, and thus

$$
\begin{gathered}
\langle\phi|\left[\phi(x)+i \int_{\Sigma} D\left(x-x^{\prime}\right) \mu\left(x^{\prime}\right)\left(d x^{\prime}\right)\right]=0 \\
\mu(x)\left|0_{\mu}\right\rangle=0
\end{gathered}
$$

where both $\phi(x)$ and $\mu\left(x^{\prime}\right)$ are now the matrices defined by (5.18) and (5.19).

The equations (5.24-26) give a complete description of the expectation value of any functional $Q(\phi)$, which is given as the matrix element of an equivalent matrix $Q(\phi)$ between the prescribed Hilbert vectors $\langle\phi|$ and $\left|0_{\mu}\right\rangle$.

The representations of $\phi(x)$ and $\mu(x)$ in (5.18) and (5.19) are the special representations in which $\mu[\Sigma]$ is diagonal. However, we could use various representations by suitable transformations, for which the commutation relations (5.21) and the equations (5.24-26) will remain the same. For instance, the representations in which $\phi[\Sigma]$ is diagonal are obtained by the use of the transformation function given by (5.11), and then (5.24) becomes

$$
\begin{aligned}
\operatorname{Av}[Q(\phi)] & =\iint\left\langle\phi \mid \phi[\Sigma]^{\prime}\right\rangle d \phi[\Sigma]^{\prime}\left\langle\phi[\Sigma]^{\prime}|Q(\phi)| \phi[\Sigma]^{\prime \prime}\right\rangle d \phi[\Sigma]^{\prime \prime}\left\langle\phi[\Sigma]^{\prime \prime} \mid 0_{\mu}\right\rangle \\
& =\int\left\langle\phi \mid \phi[\Sigma]^{\prime}\right\rangle Q\left(\phi^{\prime}\right) d \phi[\Sigma]^{\prime}, \quad\left\langle\phi[\Sigma]^{\prime \prime} \mid 0_{\mu}\right\rangle=1,
\end{aligned}
$$

${ }^{9}$ Equation (5.21) is derived from the identity

$$
\mu(x)=\int_{\Sigma} \delta\left(x-x^{\prime}\right) \mu\left(x^{\prime}\right)\left(d x^{\prime}\right)
$$


which turns out to be the original definition of the expectation value.

The equation (5.26a), for $\langle\phi|$ can be replaced by a simple condition corresponding to (5.26b) for $\left|0_{\mu}\right\rangle$ as follows. We put

$$
\langle\phi|=\left\langle 0_{\phi}\right| e^{-G[\Sigma]}
$$

with

$$
G[\Sigma]=\frac{1}{2} \iint_{\Sigma} \mu(x) D\left(x-x^{\prime}\right) \mu\left(x^{\prime}\right)(d x)\left(d x^{\prime}\right)
$$

and substitute in (5.26a), and then operate $e^{+G[\Sigma]}$ from the right side. Here, using the lemma ${ }^{10}$

$$
e^{-G} Q e^{+G}=Q-[G, Q]+\frac{1}{2 !}[G,[G, Q]]-\frac{1}{3 !}[G,[G,[G, Q]]]+\ldots
$$

and also the commutation relations (5.21), we see that

$$
\begin{aligned}
& e^{-G[\Sigma]} \phi(x) e^{G[\Sigma]}=\phi(x)-i \int_{\Sigma} D\left(x-x^{\prime}\right) \mu\left(x^{\prime}\right)\left(d x^{\prime}\right) \equiv \phi(x), \\
& e^{-G[\Sigma]} \mu(x) e^{G[\Sigma]}=\mu(x),
\end{aligned}
$$

since the terms of the orders higher than the second in (5.30) vanish for $Q=\phi(x)$. Thus, the condition for $\left\langle 0_{\phi}\right|$, corresponding to $(5.26 \mathrm{~b})$, turns out to be

$$
\left\langle 0_{\phi}\right| \phi(x)=0,\left\langle 0_{\phi} \mid 0_{\mu}\right\rangle=1,
$$

which means that $\left\langle 0_{\phi}\right|$ is a left-hand eigenvector of $\phi$ having the eigenvalue $\phi^{\prime}=0$.

On the other hand, using (5.28) in (5.24) and taking into account the condition (5.26b) and thus $G[\Sigma]\left|0_{\mu}\right\rangle=0$,

$$
\begin{aligned}
\operatorname{Av}[Q(\phi)] & =\left\langle 0_{\phi}\left|e^{-Q[\Sigma]} Q(\phi) e^{G[\Sigma]}\right| 0_{\mu}\right\rangle \\
& =\left\langle 0_{\phi}|Q(\phi)| 0_{\mu}\right\rangle, \quad\left\langle 0_{\phi} \mid 0_{\mu}\right\rangle=1,
\end{aligned}
$$

where $\dot{\phi}(x)$ is defined in (5.31) and

$$
e^{-G[\Sigma]} \phi(x)^{n} e^{G[\Sigma]}=\phi(x)^{n}, \quad e^{-G[\Sigma]} Q(\phi) e^{G[\Sigma]}=Q(\phi)
$$

for any functional $Q(\phi)$ expandable with respect to $\phi$. Equation (5.33) gives the expectation value of any $Q$ as the matrix element of the corresponding matrix representation between the Hilbert vectors $\left\langle 0_{\phi}\right|$ and $\left|0_{\mu}\right\rangle$.

It is very convenient to replace $\mu(x)$ by $\mu(x)+q(x)$, where $q(x)$ is an arbitrary ordinary real function and will be called "the external source" of the medium in the following. Thus, $G[\Sigma]$ of (5.29) and $\phi(x)$ of (5.31) are respectively replaced by

$$
G[\Sigma]=\frac{1}{2} \iint_{\Sigma}(\mu(x)+q(x)) D\left(x-x^{\prime}\right)\left(\mu\left(x^{\prime}\right)+q\left(x^{\prime}\right)\right)(d x)\left(d x^{\prime}\right),
$$

${ }^{10}$ Equation (5.30) is derived from the expansion

$$
e^{-a G} Q e^{a G}=\sum_{n=0}^{\infty} \frac{a^{n}}{n !} Q^{(n)}, \quad Q^{(0)}=Q,
$$

$a$ being an ordinary number, where $Q^{(n)}$ 's are obtained by successive differentiation of both sides with respect to $a$ at $a=1$. Added in proof: This transformation is to be understood as a similarity transformation. 


$$
\begin{aligned}
\phi(x) & =e^{-G[\Sigma]} \phi(x) e^{G[\Sigma]}=\phi(x)-i \int_{\Sigma} D\left(x-x^{\prime}\right)\left(\mu\left(x^{\prime}\right)+q\left(x^{\prime}\right)\right)\left(d x^{\prime}\right), \\
\langle\phi(x)\rangle_{0} & \equiv\left\langle 0_{\phi}|\phi(x)| 0_{\mu}\right\rangle=-i \int_{\Sigma} D\left(x-x^{\prime}\right) q\left(x^{\prime}\right)\left(d x^{\prime}\right)
\end{aligned}
$$

in terms of the notation

$$
\langle F\rangle_{0} \equiv\left\langle 0_{\phi}|F| 0_{\mu}\right\rangle
$$

Now, for the arbitrary functional $Q(\phi)$, we consider, on using the conditions for $\left\langle 0_{\phi}\right|$ and $\left|0_{\mu}\right\rangle$, i.e., (5.32) and (5.26b),

$$
\begin{aligned}
\langle\phi(x) Q(\phi)\rangle_{0} & =\langle Q(\phi) \phi(x)\rangle_{0} \\
& =\left\langle Q(\phi)\left\{\phi(x)+\langle\phi(x)\rangle_{0}\right\}\right\rangle_{0} \\
& =\langle[Q(\phi), \phi(x)]\rangle_{0}+\langle\phi(x)\rangle_{0}\langle Q(\phi)\rangle_{0},
\end{aligned}
$$

where, using the commutation relation (5.21),

$$
\begin{aligned}
{[Q(\phi), \phi(x)] } & =-[\phi(x), Q(\phi)]=i(\delta / \delta \mu(x)) Q(\phi) \\
& =i(\delta / \delta q(x)) Q(\phi),
\end{aligned}
$$

since the dependence of $\mu(x)$ on $\phi(x)$ is the same as that of $q(x)$. Thus, (5.36) becomes

$$
\langle\phi(x) Q(\phi)\rangle_{0}=\left\{\langle\phi(x)\rangle_{0}+i(\delta / \delta q(x))\right\}\langle Q(\phi)\rangle_{0} .
$$

On using (5.35), (5.38) yields for $q=0$

$$
\langle\phi(x) Q(\phi)\rangle_{0}=\int_{\Sigma} D\left(x-x^{\prime}\right)\left\langle\left(\delta / \delta \phi\left(x^{\prime}\right)\right) Q(\phi)\right\rangle_{0}\left(d x^{\prime}\right),
$$

which turns out to be the equation equivalent to (I.5.18).

From (5.38), we observe that

or, more generally,

$$
\left\langle\phi(x)^{n}\right\rangle_{0}=\left\{\langle\phi(x)\rangle_{0}+i(\delta / \delta q(x))\right\}^{n}, \quad\left\langle 0_{\phi} \mid 0_{\mu}\right\rangle=1,
$$

$$
\langle Q(\phi)\rangle_{0}=Q\left(\langle\phi(x)\rangle_{0}+i(\delta / \delta q(x))\right),
$$

in which we readily find, e.g.,

$$
\left.\left\langle\phi(x) \phi\left(x^{\prime}\right)\right\rangle_{0}\right|_{q=0}=D\left(x-x^{\prime}\right) .
$$

On the other hand, we observe from (5.38) that, on reference to (5.35),

$$
\begin{aligned}
& i(\delta / \delta q(x))\left\{e^{-G_{0}[\Sigma]}\langle Q(\phi)\rangle_{0}\right\}=e^{-G_{0}[\Sigma]^{\prime}}\langle\phi(x) Q(\phi)\rangle_{0}, \\
& G_{0}[\Sigma]=\left.G[\Sigma]\right|_{\mu=0},
\end{aligned}
$$

and thus, generally,

$$
(i(\delta / \delta q(x)))^{n}\left\{e^{-G_{0}[\Sigma]}\langle Q(\phi)\rangle_{0}\right\}=e^{-G_{0}[\Sigma]}\left\langle\phi(x)^{n} Q(\phi)\right\rangle_{0}
$$


Therefore, according to Taylor expansion,

$$
\begin{aligned}
& \left.e^{-G_{0}[\Sigma]}\langle Q(\phi)\rangle_{0}\right|_{q=q^{\prime}}=\left.\sum_{n=0}^{\infty} \frac{1}{n !}\left\{\int_{\Sigma} q(x)^{\prime} \frac{\delta}{\delta q(x)}(d x)\right\}^{n} e^{-G_{0}[\Sigma]}\langle Q(\phi)\rangle_{0}\right|_{q=0} \\
& =\left.\sum_{n=0}^{\infty} \frac{1}{n !} e^{-G_{0}[\Sigma]}\left\langle\left\{-i \int_{\Sigma} q(x)^{\prime} \phi(x)(d x)\right\}^{n} Q(\phi)\right\rangle_{0}\right|_{q=0} \\
& =\left.\left\langle\exp \left[-i \int_{\Sigma} q(x)^{\prime} \phi(x)(d x)\right] Q(\phi)\right\rangle_{0}\right|_{q=0},\left.G_{0}[\Sigma]\right|_{q=0}=0,
\end{aligned}
$$

in view of (5.43). Hence, we finally have, in terms of the convention $\left.G_{0}[\Sigma]^{\prime} \equiv G_{0}[\Sigma]\right|_{q=q^{\prime}}$,

$$
\left.\langle Q(\phi)\rangle_{0}\right|_{q=q^{\prime}}=\left.e^{+G_{0}[\Sigma]}\left\langle\exp \left[-i \int_{\Sigma} \phi(x) q(x)^{\prime}(d x)\right] Q(\phi)\right\rangle_{0}\right|_{q=0},
$$

which gives the explicit dependence of $\langle Q(\phi)\rangle_{0}$ on the external source $q(x)^{\prime}$. In the special case of $Q=1,(5.45)$ shows

$$
\left\langle\exp \left[-i \int_{\Sigma} \phi(x) q(x)^{\prime}(d x)\right]\right\rangle_{0}=e^{-G_{0}[\Sigma]^{\prime}},
$$

which is the generating function giving

$$
\left\langle\boldsymbol{\phi}\left(x_{1}\right) \phi\left(x_{2}\right) \ldots \phi\left(x_{n}\right)\right\rangle_{0}=\left.\left(i \delta / \delta q\left(x_{1}\right)\right)\left(i \delta / \delta q\left(x_{2}\right)\right) \ldots\left(i \delta / \delta q\left(x_{n}\right)\right) \exp \left[-G_{0}[\Sigma]\right]\right|_{q=0}
$$

On the other hand, differentiating both sides of (5.45) with respect to $q(x)^{\prime}$, we observe

$$
\left.i\left(\delta / \delta q(x)^{\prime}\right)\langle Q(\phi)\rangle_{0}\right|_{q=q^{\prime}}=\langle\phi(x) Q(\phi)\rangle_{0}-\left.\langle\phi(x)\rangle_{0}\langle Q(\phi)\rangle_{0}\right|_{q=q^{\prime}}
$$

reproducing (5.38) again. As the special case,

$$
i(\delta / \delta q(x))\left\langle\boldsymbol{\phi}\left(x^{\prime}\right)\right\rangle_{0}=\left\langle\boldsymbol{\phi}(x) \boldsymbol{\phi}\left(x^{\prime}\right)\right\rangle_{0}-\langle\boldsymbol{\phi}(x)\rangle_{0}\left\langle\boldsymbol{\phi}\left(x^{\prime}\right)\right\rangle_{0},
$$

on dropping the prime of $q(x)^{\prime}$.

\subsection{Mathematical Preparation for Matrix Representation of Wave Variables}

According to the expression (5.7) of the probability density function of waves, this can be given, on using (5.33), in the form

Here

$$
\left\langle\psi\left[\sigma_{1}\right]^{\prime}, \sigma_{1} \mid \psi\left[\sigma_{2}\right]^{\prime}, \sigma_{2}\right\rangle=\left\langle 0_{\phi}\left|\left\langle\psi\left[\sigma_{1}\right]^{\prime}, \sigma_{1} \mid \psi\left[\sigma_{2}\right]^{\prime}, \sigma_{2}\right\rangle\right| 0_{\mu}\right\rangle
$$

$$
\begin{aligned}
& \left\langle\psi\left[\sigma_{1}\right]^{\prime}, \sigma_{1} \mid \psi\left[\sigma_{2}\right]^{\prime}, \sigma_{2}\right\rangle=\int\left(d \bar{\psi}\left[\sigma_{2}\right]^{\prime} / 2 \pi\right) \exp \left[\frac{1}{2}\left(A-A^{*}\right)\right], \\
& A=\left.A\left(\psi\left[\sigma_{1}\right]^{\prime}, \sigma_{1} \mid \psi\left[\sigma_{2}\right]^{\prime}, \sigma_{2}\right)\right|_{\phi[\Sigma] \rightarrow \phi[\Sigma],} \\
& \left(d \bar{\psi}[\sigma]^{\prime} / 2 \pi\right)=\Pi_{a}\left(d \bar{\psi}_{a}[\sigma]^{\prime} / 2 \pi\right)_{R}\left(d \bar{\psi}_{a}[\sigma]^{\prime} / 2 \pi\right)_{l},
\end{aligned}
$$

and $\psi[\sigma]$ stands for the implicit dependence of $\psi[\sigma]$ on the operator $\phi[\Sigma]$, so that the bracket $\left\langle\psi\left[\sigma_{1}\right]^{\prime}, \sigma_{1} \mid \psi\left[\sigma_{2}\right]^{\prime}, \sigma_{2}\right\rangle$ is still an operator due to the $\phi[\Sigma]$ involved. Thus, for instance, the representation, in which $\phi[\Sigma]$ is diagonal, $(5.50 \mathrm{a})$ gives

$$
\left\langle\psi\left[\sigma_{1}\right]^{\prime}, \sigma_{1} \mid \psi\left[\sigma_{2}\right]^{\prime \prime}, \sigma_{2}\right\rangle=\iint\left\langle 0_{\phi} \mid \phi[\Sigma]^{\prime}\right\rangle d \phi[\Sigma]^{\prime}\left\langle\zeta\left[\sigma_{1}\right]^{\prime}, \sigma_{1} \mid \zeta\left[\sigma_{2}\right]^{\prime \prime}, \sigma_{2}\right\rangle d \phi[\Sigma]^{\prime \prime}\left\langle\phi[\Sigma]^{\prime \prime} \mid 0_{\mu}\right\rangle .
$$

\footnotetext{
${ }^{11}$ Indeed, when $q=0$, (5.5la) leads to the original definition of the average value, since $\left\langle\phi[\Sigma]^{\prime \prime} \mid 0_{\mu}\right\rangle=1$, while $\left\langle 0_{\phi} \mid \phi[\Sigma]^{\prime}\right\rangle$ turns out to be the given probability density function, yielding the probability $\left\langle 0_{\phi} \mid \phi[\Sigma]^{\prime}\right\rangle d \phi[\Sigma]^{\prime}$ that $\phi[\Sigma]$ has a value between $\phi[\Sigma]^{\prime}$ and $\phi[\Sigma]^{\prime}+d \phi[\Sigma]^{\prime}$.
} 
Here

$$
\left\langle\zeta\left[\sigma_{1}\right]^{\prime}, \sigma_{1} \mid \zeta\left[\sigma_{2}\right]^{\prime \prime}, \sigma_{2}\right\rangle=\left.\left\langle\psi\left[\sigma_{1}\right]^{\prime}, \sigma_{1} \mid \psi\left[\sigma_{2}\right]^{\prime \prime}, \sigma_{2}\right\rangle\right|_{\phi=\phi^{\prime}} \delta\left(\phi[\Sigma]^{\prime}-\phi[\Sigma]^{\prime \prime}\right),
$$

and $\zeta\left[\sigma_{1}\right]^{\prime}$ stands for both $\psi\left[\sigma_{1}\right]^{\prime}$ and $\phi[\Sigma]^{\prime}$, and $\zeta\left[\sigma_{2}\right]^{\prime \prime}$ for both $\psi\left[\sigma_{2}\right]^{\prime \prime}$ and $\phi[\Sigma]^{\prime \prime}$. In the special case of $\sigma_{1}=\sigma_{2}=\sigma$,

$$
\left\langle\zeta[\sigma]^{\prime}, \sigma \mid \zeta[\sigma]^{\prime \prime}, \sigma\right\rangle=\delta\left(\psi[\sigma]^{\prime}-\psi[\sigma]^{\prime \prime}\right) \delta\left(\phi[\Sigma]^{\prime}-\phi[\Sigma]^{\prime \prime}\right),
$$

which is evident from (5.6). The bracket term defined by (5.51b) will be called the Transformation Function in the following.

For later convenience, we also introduce the following notation for an arbitrary functional $Q$ of $\psi[\sigma]^{\prime}, \bar{\psi}[\sigma]^{\prime}$, and $\phi[\Sigma]^{\prime}\left(x_{3}\left[\sigma_{1}\right] \geqslant x_{3}[\sigma] \geqslant x_{3}\left[\sigma_{2}\right]\right)$;

$$
\left\langle\zeta\left[\sigma_{1}\right]^{\prime}, \sigma_{1}|Q(\psi, \bar{\psi}, \phi)| \zeta\left[\sigma_{2}\right]^{\prime \prime}, \sigma_{2}\right\rangle \equiv \int\left(d \bar{\psi}\left[\sigma_{2}\right]^{\prime} / 2 \pi\right) Q\left(\psi^{\prime}, \bar{\psi}^{\prime}, \boldsymbol{\phi}^{\prime}\right) \exp \left[\frac{1}{2}\left(A-A^{*}\right)\right] \delta\left(\phi[\Sigma]^{\prime}-\phi[\Sigma]^{\prime \prime}\right) .
$$

Here, $\psi^{\prime}$ and $\bar{\psi}^{\prime}$ are the solutions of the wave equations with the boundary values of $\psi\left[\sigma_{1}\right]^{\prime}$ (or $\psi\left[\sigma_{2}\right]^{\prime \prime}$ ) and $\bar{\psi}\left[\sigma_{2}\right]^{\prime}$, respectively, according to (2.37) and (2.38); therefore, they can be defined also by the use of $\psi(x)^{\prime}=-\delta A / \delta \bar{\eta}(x)$ and $\bar{\psi}(x)^{\prime}=-\delta A / \delta \eta(x)$ according to (4.8) with (4.4).

It is noticed that there is no prime for the arguments of $Q$ on the left side. Also, in order to be self-consistent, the order of the variables in $Q$ on the left side is very important and must be "wellordered," as will be seen later.

In the special cases of $Q=1, \phi(x)$, or $\psi\left[\sigma_{1}\right]$ (or $\psi\left[\sigma_{2}\right]$ ), we find, from (5.52), that

$$
\left\langle\zeta\left[\sigma_{1}\right]^{\prime}, \sigma_{1}|1| \zeta\left[\sigma_{2}\right]^{\prime \prime}, \sigma_{2}\right\rangle=\left\langle\zeta\left[\sigma_{1}\right]^{\prime}, \sigma_{1} \mid \zeta\left[\sigma_{2}\right]^{\prime \prime}, \sigma_{2}\right\rangle,
$$

$$
\begin{aligned}
& \left\langle\zeta\left[\sigma_{1}\right]^{\prime}, \sigma_{1}\left|\psi\left[\sigma_{1}\right]\right| \zeta\left[\sigma_{2}\right]^{\prime \prime}, \sigma_{2}\right\rangle=\psi\left[\sigma_{1}\right]^{\prime}\left\langle\zeta\left[\sigma_{1}\right]^{\prime}, \sigma_{1} \mid \zeta\left[\sigma_{2}\right]^{\prime \prime}, \sigma_{2}\right\rangle, \\
& \left\langle\zeta\left[\sigma_{1}\right]^{\prime}, \sigma_{1}|\phi(x)| \zeta\left[\sigma_{2}\right]^{\prime \prime}, \sigma_{2}\right\rangle=\phi(x)^{\prime}\left\langle\zeta\left[\sigma_{1}\right]^{\prime}, \sigma_{1} \mid \zeta\left[\sigma_{2}\right]^{\prime \prime}, \sigma_{2}\right\rangle,
\end{aligned}
$$

and a similar equation result for $\psi\left[\sigma_{2}\right]$, instead of $\psi\left[\sigma_{1}\right]$.

Now we investigate the change of $\left\langle\zeta\left[\sigma_{1}\right]^{\prime}, \sigma_{1} \mid \zeta\left[\sigma_{2}\right]^{\prime}, \sigma_{2}\right\rangle$ due to the variations of $\zeta\left[\sigma_{1}\right]^{\prime}, \zeta\left[\sigma_{2}\right]^{\prime}$, $x_{3}\left[\sigma_{1}\right], x_{3}\left[\sigma_{2}\right]$, and also due to those of $\eta$ and $\bar{\eta}$; on reference to the first equation of (5.53) with (5.52),

$$
\delta\left\langle\zeta\left[\sigma_{1}\right]^{\prime}, \sigma_{1} \mid \zeta\left[\sigma_{2}\right]^{\prime}, \sigma_{2}\right\rangle=\left\langle\zeta\left[\sigma_{1}\right]^{\prime}, \sigma_{1}\left|\frac{1}{2}\left(\delta A-\delta A^{*}\right)\right| \zeta\left[\sigma_{2}\right]^{\prime}, \sigma_{2}\right\rangle,
$$

where, from (4.8) with (4.4) and (5.8),

$$
\begin{aligned}
\delta A & =i \int_{\sigma_{1}} \bar{\psi} \gamma_{3} \delta \psi d \sigma_{3}+P_{3}\left[\sigma_{1}\right] \delta x_{3}\left[\sigma_{1}\right]-i \int_{\sigma_{2}} \bar{\psi} \gamma_{3} \delta \psi d \sigma_{3}-P_{3}\left[\sigma_{2}\right] \delta x_{3}\left[\sigma_{2}\right]+\int_{\sigma_{2}}^{\sigma_{1}} \delta L(d x), \\
\delta L(x) & =-\{\bar{\psi}(x) \delta \eta(x)+\delta \bar{\eta}(x) \psi(x)\}-\{\bar{\psi}(x) \gamma \psi(x)\} \delta \phi(x),
\end{aligned}
$$

and the variation due to $\delta \phi$ is added for later convenience.

\section{Introduction of Matrix Representation of Wave Variables and Commutation Relations}

In (5.54) combined with (5.55), we first consider the variation of $\left\langle\zeta\left[\sigma_{1}\right]^{\prime}, \sigma_{1} \mid \zeta\left[\sigma_{2}\right]^{\prime} \sigma_{2}\right\rangle$ due to $\delta \psi_{a}\left[\sigma_{1}\right]^{\prime}$ (where the Latin subscript is attached to express the components explicitly) or the corresponding variation $\delta \psi_{\alpha}\left(\sigma_{1}(x)\right)^{\prime}$. According to the definition of the $\langle>$-notation of (5.52), the $\delta \psi_{\alpha}\left(\sigma_{1}(x)\right)$ contained in $\delta A$ on the right side of (5.54) can be taken out of $\langle>$ and primed, as in (5.53). Hence 


$$
\begin{aligned}
\delta\left\langle\zeta\left[\sigma_{1}\right]^{\prime}, \sigma_{1} \mid \zeta\left[\sigma_{2}\right]^{\prime}, \sigma_{2}\right\rangle & =i \int_{\sigma_{1}} d \sigma_{3}(x)\left\langle\zeta\left[\sigma_{1}\right]^{\prime}, \sigma_{1}\left|\frac{1}{2}\left\{\bar{\psi}(x) \gamma_{3}\right\}_{\alpha}\right| \zeta\left[\sigma_{2}\right]^{\prime}, \sigma_{2}\right\rangle \delta \psi_{\alpha}\left(\sigma_{1}(x)\right)^{\prime} \\
& =i\left\langle\zeta\left[\sigma_{1}\right]^{\prime}, \sigma_{1}\left|\frac{1}{2}\left\{\bar{\psi}\left[\sigma_{1}\right] N\right\}_{a}\right| \zeta\left[\sigma_{2}\right]^{\prime}, \sigma_{2}\right\rangle \delta \psi_{a}\left[\sigma_{1}\right]^{\prime}
\end{aligned}
$$

the latter of which is obtained on reference to (4.14). Since (6.1a) is true for arbitrary $\delta \psi_{\alpha}\left(\sigma_{1}(x)\right)^{\prime}$, it follows that

$$
-i\left(\delta / \delta \psi_{\alpha}\left(\sigma_{1}(x)\right)^{\prime}\right)\left\langle\zeta\left[\sigma_{1}\right]^{\prime}, \sigma_{1} \mid \zeta\left[\sigma_{2}\right]^{\prime}, \sigma_{2}\right\rangle=\left\langle\zeta\left[\sigma_{1}\right]^{\prime}, \sigma_{1}\left|\frac{1}{2}\left\{\bar{\psi}\left(\sigma_{1}(x)\right) \gamma_{3}\right\}_{\alpha}\right| \zeta\left[\sigma_{2}\right]^{\prime}, \sigma_{2}\right\rangle
$$

or

$$
-i\left(\delta / \delta \psi_{a}\left[\sigma_{1}\right]^{\prime}\right)\left\langle\zeta\left[\sigma_{1}\right]^{\prime}, \sigma_{1} \mid \zeta\left[\sigma_{2}\right]^{\prime}, \sigma_{2}\right\rangle=\left\langle\zeta\left[\sigma_{1}\right]^{\prime}, \sigma_{1}\left|\frac{1}{2}\left\{\bar{\psi}\left[\sigma_{1}\right] N\right\}_{a}\right| \zeta\left[\sigma_{2}\right]^{\prime}, \sigma_{2}\right\rangle
$$

In the same way, we obtain

$$
\begin{aligned}
& +i\left(\delta / \delta \psi_{\alpha}\left(\sigma_{2}(x)\right)^{\prime}\right)\left\langle\zeta\left[\sigma_{1}\right]^{\prime}, \sigma_{1} \mid \zeta\left[\sigma_{2}\right]^{\prime}, \sigma_{2}\right\rangle=\left\langle\zeta\left[\sigma_{1}\right]^{\prime}, \sigma_{1}\left|\frac{1}{2}\left\{\bar{\psi}\left(\sigma_{2}(x)\right) \gamma_{3}\right\}_{\alpha}\right| \zeta\left[\sigma_{2}\right]^{\prime}, \sigma_{2}\right\rangle, \\
& +i\left(\delta / \delta \psi_{a}\left[\sigma_{2}\right]^{\prime}\right)\left\langle\zeta\left[\sigma_{1}\right]^{\prime}, \sigma_{1} \mid \zeta\left[\sigma_{2}\right]^{\prime}, \sigma_{2}\right\rangle=\left\langle\zeta\left[\sigma_{1}\right]^{\prime}, \sigma_{1}\left|\frac{1}{2}\left\{\bar{\psi}\left[\sigma_{2}\right] N\right\}_{a}\right| \zeta\left[\sigma_{2}\right]^{\prime}, \sigma_{2}\right\rangle,
\end{aligned}
$$

and similar equations for the complex conjugate variables.

For the variation due to the displacements $\delta x_{3}\left[\sigma_{1}\right]$ and $\delta x_{3}\left[\sigma_{2}\right],(5.54)$ gives, with reference to $(5.55)$,

$$
\begin{aligned}
& i\left(\partial / \partial x_{3}\left[\sigma_{1}\right]\right)\left\langle\zeta\left[\sigma_{1}\right]^{\prime}, \sigma_{1} \mid \zeta\left[\sigma_{2}\right]^{\prime} \sigma_{2}\right\rangle=\left\langle\zeta\left[\sigma_{1}\right]^{\prime}, \sigma_{1}\left|\mathscr{H}\left[\sigma_{1}\right]\right| \zeta\left[\sigma_{2}\right]^{\prime}, \sigma_{2}\right\rangle, \\
& -i\left(\partial / \partial x_{3}\left[\sigma_{2}\right]\right)\left\langle\zeta\left[\sigma_{1}\right]^{\prime}, \sigma_{1} \mid \zeta\left[\sigma_{2}\right]^{\prime}, \sigma_{2}\right\rangle=\left\langle\zeta\left[\sigma_{1}\right]^{\prime}, \sigma_{1}\left|\mathscr{H}\left[\sigma_{2}\right]\right| \zeta\left[\sigma_{2}\right]^{\prime}, \sigma_{2}\right\rangle .
\end{aligned}
$$

Here, from (4.7b),

$$
\begin{aligned}
\mathscr{H}[\sigma] & =\frac{i}{2}\left\{P_{3}[\sigma]-P_{3}^{*}[\sigma]\right\} \\
& =\frac{i}{2} \int_{\sigma} d \sigma_{3}\left[\left\{\left(\left(\gamma \partial_{T}\right)-k\right) \psi-\eta\right\}\left(\bar{\psi} \gamma_{3}^{2}\right)-\left\{\left(\left(\gamma \partial_{T}\right)-k^{*}\right) \psi^{*}-\eta^{*}\right\}\left(\bar{\psi}^{*} \gamma_{3}^{2}\right)-\bar{\eta} \psi+\bar{\eta}^{*} \psi^{*}\right],
\end{aligned}
$$

where the order of $\bar{\psi}$ and $\psi$ is interchanged for later convenience. The superscript $T$ stands for the transposition of the matrix referred to, $\gamma_{i}^{*}=\gamma_{i}^{T}=-\gamma_{i}$, and $k^{*}=k^{T}$.

In the special case of $\sigma_{1}=\sigma_{2}=\sigma,(6.1 \mathrm{~b})$ and $(6.2 \mathrm{a})$ give, on referring to $(5.51 \mathrm{c})$,

$$
\begin{aligned}
& \left\langle\zeta[\sigma]^{\prime}\left|\frac{1}{2}\left\{\bar{\psi}(\sigma(x)) \gamma_{3}\right\}_{\alpha}\right| \zeta[\sigma]^{\prime \prime}\right\rangle=-i\left(\delta / \delta \psi_{\alpha}(\sigma(x))^{\prime}\right) \delta\left(\psi[\sigma]^{\prime}-\psi[\sigma]^{\prime \prime}\right) \delta\left(\phi[\Sigma]^{\prime}-\phi[\Sigma]^{\prime \prime}\right), \\
& \left\langle\zeta[\sigma]^{\prime}\left|\frac{1}{2}\{\bar{\psi}[\sigma] N\}_{a}\right| \zeta[\sigma]^{\prime \prime}\right\rangle=-i\left(\delta / \delta \psi_{a}[\sigma]^{\prime}\right) \delta\left(\psi[\sigma]^{\prime}-\psi[\sigma]^{\prime \prime}\right) \delta\left(\phi[\Sigma]^{\prime}-\phi[\Sigma]^{\prime \prime}\right),
\end{aligned}
$$

where $\sigma$ is omitted in the above angle brackets on the left side since there is no explicit dependence on it.

Now we introduce the "matrices" $\frac{1}{2}\left\{\bar{\psi}(\sigma(x)) \gamma_{3}\right\}_{\alpha}$ and $\frac{1}{2}\{\bar{\psi}[\sigma] N\}_{a}$ whose matrix elements $\left\langle\zeta[\sigma]^{\prime}\left|\frac{1}{2}\left\{\bar{\psi}(\sigma(x)) \gamma_{3}\right\}_{\alpha}\right| \zeta[\sigma]^{\prime \prime}\right\rangle$ and $\left\langle\zeta[\sigma]^{\prime}\left|\frac{1}{2}\{\bar{\psi}[\sigma] N\}_{a}\right| \zeta[\sigma]^{\prime \prime}\right\rangle$ are defined by those in (6.5), respectively; these constitute the ordinary matrix representations of the differential operators $-i\left(\delta / \delta \psi_{\alpha}(\sigma(x))^{\prime}\right)$ and $-i\left(\delta / \delta \psi_{a}[\sigma]^{\prime}\right)$, respectively, and therefore, for instance, $(6.2 \mathrm{a})$ and $(6.2 \mathrm{~b})$ for $\sigma_{1} \neq \sigma_{2}$ can be represented as

$$
\begin{aligned}
& \int\left\langle\zeta\left[\sigma_{1}\right]^{\prime}\left|\frac{1}{2}\left\{\bar{\psi}\left(\sigma_{1}(x)\right) \gamma_{3}\right\}_{\alpha}\right| \zeta\left[\sigma_{1}\right]^{\prime \prime}\right\rangle\left(d \zeta\left[\sigma_{1}\right]^{\prime \prime}\right)\left\langle\zeta\left|\left[\sigma_{1}\right]^{\prime \prime}, \sigma_{1}\right| \zeta\left[\sigma_{2}\right]^{\prime}, \sigma_{2}\right\rangle \\
& =\left\langle\zeta\left[\sigma_{1}\right]^{\prime}, \sigma_{1}\left|\frac{1}{2}\left\{\bar{\psi}\left(\sigma_{1}(x)\right) \gamma_{3}\right\}_{\alpha}\right| \zeta\left[\sigma_{2}\right]^{\prime}, \sigma_{2}\right\rangle, \quad\left(d \zeta[\sigma]^{\prime}\right) \equiv\left(d \psi[\sigma]^{\prime}\right) d \phi[\Sigma]^{\prime},
\end{aligned}
$$


$\int\left\langle\zeta\left[\sigma_{1}\right]^{\prime}, \sigma_{1} \mid \zeta\left[\sigma_{2}\right]^{\prime \prime}, \sigma_{2}\right\rangle\left(d \zeta\left[\sigma_{2}\right]^{\prime \prime}\right)\left\langle\zeta\left[\sigma_{2}\right]^{\prime \prime}\left|\frac{1}{2}\left\{\bar{\psi}\left(\sigma_{2}(x)\right) \gamma_{3}\right\}_{\alpha}\right| \zeta\left[\sigma_{2}\right]^{\prime}\right\rangle$

$$
=\left\langle\zeta\left[\sigma_{1}\right]^{\prime}, \sigma_{1}\left|\frac{1}{2}\left\{\bar{\psi}\left(\sigma_{2}(x)\right) \gamma_{3}\right\}_{\alpha}\right| \zeta\left[\sigma_{2}\right]^{\prime}, \sigma_{2}\right\rangle \text {, etc. }
$$

Thus, we infer that $\left\langle\zeta\left[\sigma_{1}\right]^{\prime}, \sigma_{1} \mid \zeta\left[\sigma_{2}\right]^{\prime}, \sigma_{2}\right\rangle$ constitutes a right-hand Hilbert vector for the operator $\frac{1}{2}\left\{\bar{\psi}\left(\sigma_{1}\right) \gamma_{3}\right\}_{\alpha}$, and a left-hand vector for $\frac{1}{2}\left\{\bar{\psi}\left(\sigma_{2}\right) \gamma_{3}\right\}_{\alpha}$.

The matrix representations (6.5) for $\frac{1}{2}\left\{\bar{\psi}(\sigma) \gamma_{3}\right\}_{\alpha}$ and $\frac{1}{2}\{\bar{\psi}[\sigma] N\}_{a}$ can be regarded as the representations in which the "matrices" $\psi_{\alpha}(\sigma)$ or $\psi_{a}[\sigma]$ (and $\psi_{\alpha}^{*}(\sigma)$ or $\psi_{a}^{*}[\sigma]$ ) and $\phi[\Sigma]$, i.e., $\zeta[\sigma]$, is diagonal:

$$
\begin{gathered}
\left\langle\zeta[\sigma]^{\prime}\left|\psi_{\alpha}(\sigma(x))\right| \zeta[\sigma]^{\prime \prime}\right\rangle=\psi_{\alpha}(\sigma(x))^{\prime} \delta\left(\psi[\sigma]^{\prime}-\psi[\sigma]^{\prime \prime}\right) \delta\left(\phi[\Sigma]^{\prime}-\phi[\Sigma]^{\prime \prime}\right), \\
{ }^{-}\left\langle\zeta[\sigma]^{\prime}\left|\psi_{a}[\sigma]\right| \zeta[\sigma]^{\prime \prime}\right\rangle=\psi_{a}[\sigma]^{\prime} \delta\left(\psi[\sigma]^{\prime}-\psi[\sigma]^{\prime \prime}\right) \delta\left(\phi[\Sigma]^{\prime}-\phi[\Sigma]^{\prime \prime}\right),
\end{gathered}
$$

these being the special case of $\sigma_{1}=\sigma_{2}=\sigma$ in (5.53). Also, the matrix $\psi^{*}$ turns out to be the Hermitian conjugate of $\psi$, as is directly seen from the representations similar to (6.7).

Therefore, using the ordinary bracket convention (5.22) for the commutator of two matrices and the matrix multiplication rule

$$
\left\langle\psi[\sigma]^{\prime}|A B| \psi[\sigma]^{\prime \prime}\right\rangle=\int\left\langle\psi[\sigma]^{\prime}|A| \psi[\sigma]^{\prime \prime \prime}\right\rangle\left(d \psi[\sigma]^{\prime \prime \prime}\right)\left\langle\psi[\sigma]^{\prime \prime \prime}|B| \psi[\sigma]^{\prime \prime}\right\rangle,
$$

we obtain, in this representation, the following commutation relations:

$$
\begin{aligned}
& {\left[\frac{1}{2}\left\{\bar{\psi}(\sigma(x)) \gamma_{3}\right\}_{\alpha},\left\{\gamma_{3} \psi\left(\sigma\left(x^{\prime}\right)\right)\right\}_{\beta}\right]=-i\left(\gamma_{3}\right)_{\beta \alpha} \delta\left(\sigma(x)-\sigma\left(x^{\prime}\right)\right),} \\
& {\left[\frac{1}{2}\{\bar{\psi}[\sigma] N\}_{a}, \psi_{b}[\sigma]\right]=-i \delta_{a b},}
\end{aligned}
$$

[the $\delta$-function being defined in (2.19d)] and also the similar Hermitian conjugate equations

$$
\begin{aligned}
& {\left[\frac{1}{2}\left\{\gamma_{3} \bar{\psi}^{*}(\sigma(x))\right\}_{\alpha},\left\{\psi^{*}(\sigma(x)) \gamma_{3}\right\}_{\beta}\right]=-i\left(\gamma_{3}\right)_{\alpha \beta} \delta\left(\sigma(x)-\sigma\left(x^{\prime}\right)\right),} \\
& {\left[\frac{1}{2}\left\{N \bar{\psi}^{*}[\sigma]\right\}_{a}, \psi_{b}^{*}[\sigma]\right]=-i \delta_{a b},}
\end{aligned}
$$

corresponding to the complex conjugate wave variables. All the other commutators vanish:

$$
\left[\psi_{a}[\sigma], \psi_{b}[\sigma]\right]=\left[\bar{\psi}_{a}[\sigma], \bar{\psi}_{b}[\sigma]\right]=\left[\psi_{a}^{*}[\sigma], \psi_{b}[\sigma]\right]=\left[\bar{\psi}_{a}[\sigma], \psi_{b}^{*}[\sigma]\right]=0 \text {, etc. }
$$

We also observe directly from (6.5) and (6.7) that the wave variables and $\phi$ commute:

$$
[\psi[\sigma], \phi[\Sigma]]=[\bar{\psi}[\sigma], \phi[\Sigma]]=0, \text { etc. }
$$

On the other hand, since $\phi$ does not commute with $\phi$ and $\mu$, separately, the wave variables $\psi, \bar{\psi}$, etc., are found not to commute with $\phi$ and $\mu$ (refer to the later equation (6.22)).

The two equations in (6.9a) are dependent and derived from each other by the use of the relation $(2.19 \mathrm{a}-\mathrm{d})$; i.e.,

$$
\begin{aligned}
& \psi(\sigma(x))=\psi_{a}(\sigma(x)) N_{a b} \psi_{b}[\sigma], \quad \bar{\psi}(\sigma(x))=\bar{\psi}_{b}[\sigma] N_{b a} \psi_{a}^{*}(\sigma(x)), \\
& \left\{\psi_{a}(\sigma(x))\right\}_{\alpha} N_{a b}\left\{\psi_{b}^{*}\left(\sigma\left(x^{\prime}\right)\right)\right\}_{\beta}=\left(\gamma_{3}\right)_{\alpha \beta} \delta\left(\sigma(x)-\sigma\left(x^{\prime}\right)\right) .
\end{aligned}
$$


Since $\psi[\sigma]$ and $\phi[\Sigma]$ are commutative, the matrix representations of all relevant variables involved are possible, sich that $\psi[\sigma], \psi^{*}[\sigma]$, and $\phi[\Sigma]$, i.e., $\zeta[\sigma]$, become simultaneously diagonal.

\subsection{Equation of Motion of Transformation Function (Schrödinger Equation)}

The equation (6.3) prescribes the spatial change of the transformation function $\left\langle\xi\left[\sigma_{1}\right]^{\prime}\right.$, $\sigma_{1}\left|\zeta\left[\sigma_{2}\right]^{\prime \prime}, \sigma_{2}\right\rangle$ with respect to the coordinates $x_{3}\left[\sigma_{1}\right]$ and $x_{3}\left[\sigma_{2}\right]$, together with the boundary condition $(5.51 \mathrm{c})$ :

$$
\left.\left\langle\zeta\left[\sigma_{1}\right]^{\prime}, \sigma_{1} \mid \zeta\left[\sigma_{2}\right]^{\prime \prime}, \sigma_{2}\right\rangle\right|_{\sigma_{1} \rightarrow \sigma_{2}}=\delta\left(\psi\left[\sigma_{1}\right]^{\prime}-\psi\left[\sigma_{2}\right]^{\prime \prime}\right) \delta\left(\phi[\Sigma]^{\prime}-\phi[\Sigma]^{\prime \prime}\right) .
$$

According to the original definition (5.52) of the bracket \langle\rangle notation, the $\psi\left[\sigma_{1}\right], \psi^{*}\left[\sigma_{1}\right]$, and $\phi$ contained in $\mathscr{H}\left[\sigma_{1}\right]$ can be taken out of the brackets and primed as in (5.53), and thus the remaining terms in the brackets contain only $\bar{\psi}\left[\sigma_{1}\right]$ and $\bar{\psi}^{*}\left[\sigma_{1}\right]$ in linear form according to (6.4).

On the other hand, for the brackets containing $\bar{\psi}\left[\sigma_{1}\right]$ and $\bar{\psi}^{*}\left[\sigma_{1}\right]$, we can use the rule (6.6) with (6.5), and the corresponding one for $\bar{\psi}^{*}\left[\sigma_{1}\right]$. Hence, in (6.3), if we substitute the expression (6.4) for $\mathscr{P}\left[\sigma_{1}\right]$ with $\psi\left[\sigma_{1}\right], \bar{\psi}\left[\sigma_{1}\right], \psi^{*}\left[\sigma_{1}\right]$, and $\bar{\psi}^{*}\left[\sigma_{1}\right]$, which are now all matrices, in the given order, $\psi\left[\sigma_{1}\right]$ and $\psi^{*}\left[\sigma_{1}\right]$ being defined by (6.7), and if we apply the ordinary matrix multiplication rule (6.8), the correct equivalence is obtained for the right side of (6.3) between the one originally defined by (5.52) and the one given in the matrix representation. Thus, (6.3) is equivalent to

$$
\begin{aligned}
& i\left(\partial / \partial x_{3}\left[\sigma_{1}\right]\right)\left\langle\zeta\left[\sigma_{1}\right]^{\prime}, \sigma_{1} \mid \zeta\left[\sigma_{2}\right]^{\prime}, \sigma_{2}\right\rangle=\int\left\langle\zeta\left[\sigma_{1}\right]^{\prime}\left|\not \mathscr{P}\left[\sigma_{1}\right]\right| \zeta\left[\sigma_{1}\right]^{\prime \prime}\right\rangle\left(d \zeta\left[\sigma_{1}\right]^{\prime \prime}\right)\left\langle\zeta\left[\sigma_{1}\right]^{\prime \prime}, \sigma_{1} \mid \zeta\left[\sigma_{2}\right]^{\prime}, \sigma_{2}\right\rangle, \\
& \left.-i\left(\partial / \partial x_{3}\left[\sigma_{2}\right]\right)\left\langle\zeta\left[\sigma_{1}\right]^{\prime}, \sigma_{1} \mid \zeta\left[\sigma_{2}\right]^{\prime}, \sigma_{2}\right\rangle=\int\left\langle\zeta\left[\sigma_{1}\right]^{\prime}, \sigma_{1} \mid \zeta\left[\sigma_{2}\right]^{\prime \prime}, \sigma_{2}\right\rangle\left(d \zeta \mid \sigma_{2}\right]^{\prime \prime}\right)\left\langle\zeta\left[\sigma_{2}\right]^{\prime \prime}|\not| g\left[\sigma_{2}\right] \mid \zeta\left[\sigma_{2}\right]^{\prime}\right\rangle,
\end{aligned}
$$

$$
(d \zeta[\sigma]) \equiv(d \psi[\sigma]) d \phi[\Sigma] .
$$

Thus, the order of the variables in $\mathscr{H}[\sigma]$ is very important because they do not generally commute. However, by the use of the commutation relations (6.9), $\mathscr{P}[\sigma]$ in (6.4) can be replaced by

$$
\begin{aligned}
\mathscr{P}[\sigma] & =\frac{i}{2} \int_{\sigma} d \sigma_{3}\left[\bar{\psi} \gamma_{3}^{2}\left\{\left[\left(\gamma \partial_{T}\right)-k\right] \psi-\eta\right\}-\bar{\psi}^{*} \cdot \gamma_{3}^{2}\left\{\left[\left(\gamma \partial_{T}\right)-k^{*}\right] \psi^{*}-\eta^{*}\right\}-\bar{\eta} \psi+\bar{\eta}^{*} \psi^{*}\right] \\
& =\frac{i}{2} \int_{\sigma} d \sigma_{3}\left[\left\{\bar{\psi}\left[-\left(\gamma \overleftarrow{\partial}_{T}\right)-k\right]-\bar{\eta}\right\} \gamma_{3}^{2} \psi-\left\{\bar{\psi}^{*}\left[-\left(\overleftarrow{\left.\gamma \check{\partial}_{T}\right)}-k^{*}\right]-\bar{\eta}^{*}\right\} \gamma_{3}^{2} \psi^{*}-\bar{\psi} \eta+\bar{\psi}^{*} \eta^{*}\right],\right.
\end{aligned}
$$

with the supplementary conditions $(4.7 \mathrm{c})$, where $k^{*}=k^{T}$ is the complex conjugate matrix. The proof is not difficult by the use of (4.7a) in (4.6).

On the other hand, the right side of (6.14) is just the Hermitian conjugation of that of (6.4), and thus $\mathscr{H}[\sigma]$ is found to be Hermitian in the representation used in which $\zeta[\sigma]$ is diagonal. Therefore, we see from (6.13), together with the boundary condition (6.12), that

$$
\left\langle\zeta\left[\sigma_{1}\right]^{\prime}, \sigma_{1} \mid \zeta\left[\sigma_{2}\right]^{\prime}, \sigma_{2}\right\rangle^{*}=\left\langle\zeta\left[\sigma_{2}\right]^{\prime}, \sigma_{2} \mid \zeta\left[\sigma_{1}\right]^{\prime}, \sigma_{1}\right\rangle
$$

provided that $\left\langle\zeta\left[\sigma_{2}\right]^{\prime}, \sigma_{2} \mid \zeta\left[\sigma_{1}\right]^{\prime}, \sigma_{1}\right\rangle$ on the right side is defined by the solution of (6.13) with the boundary condition (6.12) even for $x_{3}\left[\sigma_{2}\right]<x_{3}\left[\sigma_{1}\right]$. From (6.15), we see that, when $x_{3}\left[\sigma_{2}\right]<x_{3}\left[\sigma_{1}\right]$, $\left\langle\zeta\left[\sigma_{2}\right]^{\prime}, \sigma_{2} \mid \zeta\left[\sigma_{1}\right]^{\prime}, \sigma_{1}\right\rangle$ can be defined by the complex conjugation of $\left\langle\zeta\left[\sigma_{1}\right]^{\prime}, \sigma_{1} \mid \zeta\left[\sigma_{2}\right]^{\prime}, \sigma_{2}\right\rangle$. Further, if $\bar{\eta}=\bar{\eta}^{*}=0$, the both sides of (6.15) will be real, as may be seen from the result (5.6) in connection with the relation (3.7). 
The equation (6.13) evidently corresponds to the Schrödinger equation in quantum mechanics; the time in the latter is simply replaced by $x_{3}[\sigma]$ in the former.

\subsection{A Few Relations Between Transformation Functions}

There is the following relation between the transformation functions: for any plane $\sigma_{2}$ which is perpendicular to the $x_{3}$-axis, it holds that

$$
\left\langle\zeta\left[\sigma_{1}\right]^{\prime}, \sigma_{1} \mid \zeta\left[\sigma_{3}\right]^{\prime}, \sigma_{3}\right\rangle=\int\left\langle\zeta\left[\sigma_{1}\right]^{\prime}, \sigma_{1} \mid \zeta\left[\sigma_{2}\right]^{\prime}, \sigma_{2}\right\rangle\left(d \zeta\left[\sigma_{2}\right]^{\prime}\right)\left\langle\zeta\left[\sigma_{2}\right]^{\prime}, \sigma_{2} \mid \zeta\left[\sigma_{3}\right]^{\prime}, \sigma_{3}\right\rangle .
$$

The proof of (6.16) is given first by showing that the right side is independent of the coordinate $x_{3}\left[\sigma_{2}\right]$ and then, by putting $x_{3}\left[\sigma_{2}\right] \rightarrow x_{3}\left[\sigma_{3}\right]$, both sides are found to be equal by the use of $(6.12)$; the first step of the proof is very simple by the use of (6.13).

Applying the functional differentiation $-2 \delta / \delta \eta_{\alpha}(x)$ to both sides of (6.16), where the point $x$ is between the surfaces $\sigma_{2}$ and $\sigma_{3}$, we have, on account of (5.54) and (5.55) or the later equation (6.25),

$$
\left\langle\zeta\left[\sigma_{1}\right]^{\prime}, \sigma_{1}\left|\bar{\psi}_{\alpha}(x)\right| \zeta\left[\sigma_{3}\right]^{\prime}, \sigma_{3}\right\rangle=\int\left\langle\zeta\left[\sigma_{1}\right]^{\prime}, \sigma_{1} \mid \zeta\left[\sigma_{2}\right]^{\prime}, \sigma_{2}\right\rangle\left(d \zeta\left[\sigma_{2}\right]^{\prime}\right)\left\langle\zeta\left[\sigma_{2}\right]^{\prime}, \sigma_{2}\left|\bar{\psi}_{\alpha}(x)\right| \zeta\left[\sigma_{3}\right]^{\prime}, \sigma_{3}\right\rangle .
$$

If the surface $\sigma_{2}$ is chosen so that the point $x$ is on it, the rule (6.6a) can be used on the right side of (6.17), and thus

$$
\begin{aligned}
\left\langle\zeta\left[\sigma_{1}\right]^{\prime}, \sigma_{1}\left|\bar{\psi}_{\alpha}(x)\right| \zeta\left[\sigma_{3}\right]^{\prime},\right. & \left.\sigma_{3}\right\rangle=\iint\left\langle\zeta\left[\sigma_{1}\right]^{\prime}, \sigma_{1} \mid \zeta\left[\sigma_{2}\right]^{\prime}, \sigma_{2}\right\rangle \\
& \times\left(d \zeta\left[\sigma_{2}\right]^{\prime}\right)\left\langle\zeta\left[\sigma_{2}\right]^{\prime}\left|\bar{\psi}_{\alpha}(x)\right| \zeta\left[\sigma_{2}\right]^{\prime \prime}\right\rangle\left(d \zeta\left[\sigma_{2}\right]^{\prime \prime}\right)\left\langle\zeta\left[\sigma_{2}\right]^{\prime \prime}, \sigma_{2} \mid \zeta\left[\sigma_{3}\right]^{\prime}, \sigma_{3}\right\rangle .
\end{aligned}
$$

We could do the same things also for $\psi_{\alpha}(x), \bar{\psi}_{\alpha}^{*}(x)$, and $\psi_{\alpha}^{*}(x)$ by applying $-2\left(\delta / \delta \bar{\eta}_{\alpha}(x)\right)$, $+2\left(\delta / \delta \eta_{\alpha}^{*}(x)\right)$, and $+2\left(\delta / \delta \bar{\eta}_{\alpha}^{*}(x)\right)$, respectively. Thus, it generally follows that, for any function $F(x)$ of the wave variables $\psi(x), \bar{\psi}(x), \psi^{*}(x)$, and $\bar{\psi}^{*}(x)$ on $\sigma_{2}$,

$$
\begin{aligned}
\left\langle\zeta\left[\sigma_{1}\right]^{\prime}, \sigma_{1}|F(x)| \zeta\left[\sigma_{3}\right]^{\prime}, \sigma_{3}\right\rangle=\iint & \left\langle\zeta\left[\sigma_{1}\right]^{\prime}, \sigma_{1} \mid \zeta\left[\sigma_{2}\right]^{\prime}, \sigma_{2}\right\rangle\left(d \zeta\left[\sigma_{2}\right]^{\prime}\right) \\
& \times\left\langle\zeta\left[\sigma_{2}\right]^{\prime}|F(x)| \zeta\left[\sigma_{2}\right]^{\prime \prime}\right\rangle\left(d \zeta\left[\sigma_{2}\right]^{\prime \prime}\right)\left\langle\zeta\left[\sigma_{2}\right]^{\prime \prime}, \sigma_{2} \mid \zeta\left[\sigma_{3}\right]^{\prime}, \sigma_{3}\right\rangle .
\end{aligned}
$$

\subsection{Wave Equation in the Heisenberg Representation}

The matrix representations (6.5) and (6.7) for $\bar{\psi}(\sigma(x))$ and $\psi(\sigma(x))$, respectively, can be interpreted to be the representations in which $\psi[\sigma]$ is diagonal, the point $x$ being on $\sigma$. However, $\psi[\sigma]$ itself changes with the coordinate $x_{3}[\sigma]$, and thus, if their matrix representations were based on the variable $\psi[\sigma]$ at a fixed surface, say $\sigma=\sigma_{2}$, they would change explicitly with $x_{3}[\sigma]$. As in quantum mechanics, the former representation will be called the Schrödinger representation and the latter the Heisenberg representation.

Let $F\left(x_{1}\right)$ be an arbitrary function of wave variables $\psi\left(x_{1}\right)$ and $\bar{\psi}\left(x_{1}\right)$ on $\sigma_{1}$; then, according to (6.19), its representation in which $\zeta\left[\sigma_{2}\right]$ is diagonal can be expressed, on referring to (6.15), as

$$
\begin{aligned}
\left\langle\zeta\left[\sigma_{2}\right]^{\prime}\left|F\left(x_{1}\right)\right| \zeta\left[\sigma_{2}\right]^{\prime \prime}\right\rangle=\iint\left\langle\zeta\left[\sigma_{2}\right]^{\prime}, \sigma_{2} \mid \zeta\left[\sigma_{1}\right]^{\prime}, \sigma_{1}\right\rangle\left(d \zeta\left[\sigma_{1}\right]^{\prime}\right) & \\
& \times\left\langle\zeta\left[\sigma_{1}\right]^{\prime}\left|F\left(x_{1}\right)\right| \zeta\left[\sigma_{1}\right]^{\prime \prime}\right\rangle\left(d \zeta\left[\sigma_{1}\right]^{\prime \prime}\right)\left\langle\zeta\left[\sigma_{1}\right]^{\prime \prime}, \sigma_{1} \mid \zeta\left[\sigma_{2}\right]^{\prime \prime}, \sigma_{2}\right\rangle .
\end{aligned}
$$

Applying $i\left(\partial / \partial x_{3}\left[\sigma_{1}\right]\right)$ to both sides of $(6.20)$ and using $(6.3)$, we observe, since $\left\langle\zeta\left[\sigma_{1}\right]^{\prime}\left|F\left(x_{1}\right)\right| \zeta\left[\sigma_{1}\right]^{\prime \prime}\right\rangle$ is independent of $x_{3}\left[\sigma_{1}\right]$, that 
$i\left(\partial / \partial x_{3}\left[\sigma_{1}\right]\right)\left\langle\zeta\left[\sigma_{2}\right]^{\prime}\left|F\left(x_{1}\right)\right| \zeta\left[\sigma_{2}\right]^{\prime \prime}\right\rangle$

$=-\int\left\langle\zeta\left[\sigma_{2}\right]^{\prime}, \sigma_{2}\left|\not \mathcal{P}\left[\sigma_{1}\right]\right| \zeta\left[\sigma_{1}\right]^{\prime}, \sigma_{1}\right\rangle\left(d \zeta\left[\sigma_{1}\right]^{\prime}\right)\left\langle\zeta\left[\sigma_{1}\right]^{\prime}, \sigma_{1}\left|F\left(x_{1}\right)\right| \zeta\left[\sigma_{2}\right]^{\prime \prime}, \sigma_{2}\right\rangle$

$$
\begin{array}{r}
+\int\left\langle\zeta\left[\sigma_{2}\right]^{\prime}, \sigma_{2}\left|F\left(x_{1}\right)\right| \zeta\left[\sigma_{1}\right]^{\prime}, \sigma_{1}\right\rangle\left(d \zeta\left[\sigma_{1}\right]^{\prime}\right)\left\langle\zeta\left[\sigma_{1}\right]^{\prime}, \sigma_{1}\left|\mathscr{H}\left[\sigma_{1}\right]\right| \zeta\left[\sigma_{2}\right]^{\prime \prime}, \sigma_{2}\right\rangle \\
=\left\langle\zeta\left[\sigma_{2}\right]^{\prime}, \sigma_{2}\left|-\mathscr{P}\left[\sigma_{1}\right] F\left(x_{1}\right)+F\left(x_{1}\right) \mathscr{H}\left[\sigma_{1}\right]\right| \zeta\left[\sigma_{2}\right]^{\prime \prime}, \sigma_{2}\right\rangle
\end{array}
$$

or, in the matrix representation with the convention (5.22) for the commutators,

$$
i\left(\partial / \partial x_{3}\left[\sigma_{1}\right]\right) F\left(x_{1}\right)=\left[F\left(x_{1}\right), \mathscr{P}\left[\sigma_{1}\right]\right],
$$

which corresponds to the Heisenberg equation of motion.

For instance, if we put $F\left(x_{1}\right)=\gamma_{3} \psi\left(x_{1}\right)=\gamma_{3} \psi\left(\sigma\left(x_{1}\right)\right)$ and use the commutation relations (6.9a) and $\mathscr{H}[\sigma]$ given by (6.14), the equation (6.22) becomes

$$
i\left(\partial / \partial x_{3}\right) \gamma_{3} \psi\left(x_{1}\right)=-\gamma_{3}^{2}\left[\left\{\left(\gamma \partial_{T}\right)-k\right\} \psi\left(x_{1}\right)-\eta\left(x_{1}\right)\right], \quad \text { or } \gamma_{3}^{2}\left[\{(\gamma \partial)-k\} \psi\left(x_{1}\right)-\eta\left(x_{1}\right)\right]=0,
$$

or, using the supplementary condition $(4.7 \mathrm{c})$,

$$
[(\gamma \partial)-k] \psi\left(x_{1}\right)=\eta\left(x_{1}\right) .
$$

In the same way, putting $F\left(x_{1}\right)=\bar{\psi}\left(x_{1}\right) \gamma_{3}$,

$$
\left[\bar{\psi}\left(x_{1}\right)\{-(\stackrel{4}{\partial})-k\}-\bar{\eta}\left(x_{1}\right)\right] \gamma_{3}^{2}=0
$$

or

$$
\bar{\psi}\left(x_{1}\right)[-(\gamma \overleftarrow{\partial})-k]=\bar{\eta}\left(x_{i}\right)
$$

Equations $(6.23 \mathrm{~b})$ and $(6.24 \mathrm{~b})$ are exactly the same as the original wave equations (2.1) and (2.3), respectively. The same results are also obtained for $\psi^{*}$ and $\bar{\psi}^{*}$.

In the same way as the coordinate derivatives of the wave variables (but not of $\phi(x)$ ) with respect to $x_{3}$ are obtained by (6.22), the derivatives with respect to other external variables such as $\eta(x), \bar{\eta}(x)$, and $q(x)$ are also important, and are obtained in a similar form: using (5.54) with (5.55) we observe

$$
\begin{aligned}
& \left(\delta / \delta \eta_{\alpha}(x)\right)\left\langle\zeta\left[\sigma_{1}\right]^{\prime}, \sigma_{1} \mid \zeta\left[\sigma_{2}\right]^{\prime}, \sigma_{2}\right\rangle=-\frac{1}{2}\left\langle\zeta\left[\sigma_{1}\right]^{\prime}, \sigma_{1}\left|\bar{\psi}_{\alpha}(x)\right| \zeta\left[\sigma_{2}\right]^{\prime}, \sigma_{2}\right\rangle, \\
& \left(\delta / \delta \eta_{\alpha}^{*}(x)\right)\left\langle\zeta\left[\sigma_{1}\right]^{\prime}, \sigma_{1} \mid \zeta\left[\sigma_{2}\right]^{\prime}, \sigma_{2}\right\rangle=+\frac{1}{2}\left\langle\zeta\left[\sigma_{1}\right]^{\prime}, \sigma_{1}\left|\bar{\psi}_{\alpha}^{*}(x)\right| \zeta\left[\sigma_{2}\right]^{\prime}, \sigma_{2}\right\rangle,
\end{aligned}
$$

where the point $x$ is to be in the space between $\sigma_{1}$ and $\sigma_{2}$.

On the other hand, differentiating both sides of (6.19) with respect to $\eta\left(x^{\prime}\right)$ and using (6.25), we have, as (6.22) is derived,

$$
\begin{aligned}
\left(\delta / \delta \eta_{\alpha}\left(x^{\prime}\right)\right)\left\langle\zeta\left[\sigma_{1}\right]^{\prime}, \sigma_{1}|F(x)| \zeta\left[\sigma_{2}\right]^{\prime}, \sigma_{2}\right\rangle=-\frac{1}{2}\left\langle\zeta\left[\sigma_{1}\right]^{\prime}, \sigma_{1}\left|T\left[F(x) \bar{\psi}_{\alpha}\left(x^{\prime}\right)\right]\right| \zeta\left[\sigma_{2}\right]^{\prime}, \sigma_{2}\right\rangle \\
+\left\langle\zeta\left[\sigma_{1}\right]^{\prime}, \sigma_{1}\left|\delta^{\prime} F(x) / \delta^{\prime} \eta_{\alpha}\left(x^{\prime}\right)\right| \zeta\left[\sigma_{2}\right]^{\prime}, \sigma_{2}\right\rangle, \quad x_{3}\left[\sigma_{1}\right]>x_{3}\left[\sigma_{2}\right] .
\end{aligned}
$$

Here, $T$ stands for the coordinate ordered product

$$
T\left[A(x) B\left(x^{\prime}\right)\right]= \begin{cases}A(x) B\left(x^{\prime}\right), & x_{3}>x_{3}^{\prime}, \\ B\left(x^{\prime}\right) A(x), & x_{3}<x_{3}^{\prime},\end{cases}
$$


and $\delta^{\prime} F(x) / \delta^{\prime} \eta_{\alpha}\left(x^{\prime}\right)$ is the direct functional derivative when $F(x)$ is an explicit functional of $\eta$.

In the same way, for $x_{3}\left[\sigma_{1}\right]>x_{3}\left[\sigma_{2}\right]$,

$\left(\delta / \delta \eta_{\alpha}^{*}\left(x^{\prime}\right)\right)\left\langle\zeta\left[\sigma_{1}\right]^{\prime}, \sigma_{1}|F(x)| \zeta\left[\sigma_{2}\right]^{\prime}, \sigma_{2}\right\rangle=+\frac{1}{2}\left\langle\zeta\left[\sigma_{1}\right]^{\prime}, \sigma_{1}\left|T\left[F(x) \bar{\psi}_{\alpha}^{*}\left(x^{\prime}\right)\right]\right| \zeta\left[\sigma_{2}\right]^{\prime}, \sigma_{2}\right\rangle$

$$
+\left\langle\zeta\left[\sigma_{1}\right]^{\prime}, \sigma_{1}\left|\delta^{\prime} F(x) / \delta^{\prime} \eta_{\alpha}^{*}\left(x^{\prime}\right)\right| \zeta\left[\sigma_{2}\right]^{\prime}, \sigma_{2}\right\rangle .
$$

Generally, we observe that, for $x_{3}\left[\sigma_{1}\right]>x_{3}\left[\sigma_{2}\right]$,

$\delta\left\langle\zeta\left[\sigma_{1}\right]^{\prime}, \sigma_{1}|F(x)| \zeta\left[\sigma_{2}\right]^{\prime}, \sigma_{2}\right\rangle$

$$
=\left\langle\zeta\left[\sigma_{1}\right]^{\prime}, \sigma_{1}\left|T\left[F(x) \int_{\sigma_{2}}^{\sigma_{1}} \frac{1}{2}\left\{\delta L\left(x^{\prime}\right)-\delta L^{*}\left(x^{\prime}\right)\right\}\left(d x^{\prime}\right)\right]+\delta^{\prime} F(x)\right| \zeta\left[\sigma_{2}\right]^{\prime}, \sigma_{2}\right\rangle,
$$

where, according to (5.55) with (5.8),

$$
\frac{1}{2}\left\{\delta L(x)-\delta L^{*}(x)\right\}=-i j(x) \delta \phi(x)-\frac{1}{2}\left\{\bar{\psi}(x) \delta \eta(x)+\delta \bar{\eta}(x) \psi(x)-\bar{\psi}^{*}(x) \delta \eta^{*}(x)-\delta \bar{\eta}^{*}(x) \psi^{*}(x)\right\},
$$

with

$$
j(x)=-\frac{i}{2}\left\{\bar{\psi}(x) \gamma \psi(x)-\bar{\psi}^{*}(x) \gamma^{*} \psi^{*}(x)\right\}
$$

Equation (6.29) gives the variation of $\delta\left\langle\zeta\left[\sigma_{1}\right]^{\prime}, \sigma_{1}|F(x)| \zeta\left[\sigma_{2}\right]^{\prime}, \sigma_{2}\right\rangle$ for any wave variable $F(x)$ involved in the space between $\sigma_{1}$ and $\sigma_{2}{ }^{12}$

Since $\psi(n(x))$ and $\bar{\psi}(n(x))$ given by $(2.18 \mathrm{~b})$ are not independent variables, but functions of $\psi(\sigma(x)), \eta(x)$, and $\bar{\psi}(\sigma(x)), \bar{\eta}(x)$, respectively, special care is necessary when using (6.29) for these variables. For instance, using $(2.18 \mathrm{~b})$,

$\left(\delta / \delta \eta_{\alpha}\left(x^{\prime}\right)\right)\left\langle\zeta\left[\sigma_{1}\right]^{\prime}, \sigma_{1}\left|\psi_{\beta}(n(x))\right| \zeta\left[\sigma_{2}\right]^{\prime}, \sigma_{2}\right\rangle$

$$
=-\left\langle\zeta\left[\sigma_{1}\right]^{\prime}, \sigma_{1}\left|\frac{1}{2} T\left[\psi_{\beta}(n(x)) \bar{\psi}_{\alpha}\left(x^{\prime}\right)\right]+\left\{k_{33}(x)^{-1}\left(1-\gamma_{3}^{2}\right)\right\}_{\beta \alpha} \delta\left(x-x^{\prime}\right)\right| \zeta\left[\sigma_{2}\right]^{\prime}, \sigma_{2}\right\rangle,
$$

where the term corresponding to $\delta^{\prime} F(x)$ in $(6.29)$ does not vanish.

\section{Analogies to Quantum Electrodynamics}

The important equations obtained in the preceding sections will be as follows: from (5.26b), (5.32), (5.35a), (5.50a), and (6.22),

$$
\begin{gathered}
\left\langle\psi\left[\sigma_{1}\right]^{\prime}, \sigma_{1} \mid \psi\left[\sigma_{2}\right]^{\prime}, \sigma_{2}\right\rangle=\left\langle 0_{\phi}\left|\left\langle\psi\left[\sigma_{1}\right]^{\prime}, \sigma_{1} \mid \psi\left[\sigma_{2}\right]^{\prime}, \sigma_{2}\right\rangle\right| 0_{\mu}\right\rangle, \\
\left\langle 0_{\phi}|\phi(x)=0, \quad \mu(x)| 0_{\mu}\right\rangle=0, \quad\left\langle 0_{\phi} \mid 0_{\mu}\right\rangle=1, \\
\phi(x)=\phi(x)-i \int_{\Sigma} D\left(x-x^{\prime}\right)\left\{\mu\left(x^{\prime}\right)+q\left(x^{\prime}\right)\right\}\left(d x^{\prime}\right), \\
i\left(\partial / \partial x_{3}[\sigma]\right) F(x)=[F(x), \mathscr{H}[\sigma]] .
\end{gathered}
$$

Here $F(x)$ in (7.1d) stands for the wave variables $\psi(x), \bar{\psi}(x), \psi^{*}(x)$, and $\bar{\psi}^{*}(x)$, but not the medium variables $\phi(x), \phi(x)$, and $\mu(x)$. The total Hamiltonian $\mathscr{H}[\sigma]$ is given by (6.14) together with the conditions (4.7c) which define $\psi(n(x))$ and $\bar{\psi}(n(x))$ in terms of $\psi(\sigma(x)), \bar{\psi}(\sigma(x))$, and their tangential derivatives on the surface $\sigma$, respectively. Thus, with the conditions $(4.7 \mathrm{c}), \mathscr{H}[\sigma]$ may be given by

${ }^{12}$ At this stage, $\delta \phi(x)$ in $(6.30)$ is to be an arbitrary variation and thus independent of the $\phi(x)$ whose eigenvectors are used as the basis for the representation of the transformation function. 


$$
\begin{aligned}
& \mathscr{H}[\sigma]=\mathscr{H}_{0}[\sigma]+\mathscr{H}_{1}[\sigma], \\
& \mathscr{H}_{0}[\sigma]=\frac{i}{2} \int_{\sigma} d \sigma_{3}\left[\bar{\psi}\left\{\left(\gamma \partial_{T}\right)-k_{0}\right\} \psi-\bar{\psi}^{*}\left\{\left(\gamma \partial_{T}\right)-k_{0}^{*}\right\} \psi^{*}\right], \\
& \mathscr{H}_{1}[\sigma]=\int_{\sigma} d \sigma_{3}\left[j(x) \phi(x)-\frac{i}{2}\left\{\bar{\psi} \eta+\bar{\eta} \psi-\bar{\psi}^{*} \eta^{*}-\bar{\eta}^{*} \psi^{*}\right\}\right],
\end{aligned}
$$

where $j(x)$ is defined by (6.31), and the commutation relations of the wave variables and the medium variables involved are given by $(6.9 \mathrm{a}-\mathrm{c})$ and $(5.21)$, respectively.

In (7.1) and (7.2), we observe an important analogy to the field theory: Equation (7.1d) for the wave variables is exactly the same as the Heisenberg equation of motion in quantum mechanics due to the Hamiltonian $\mathscr{P}[\sigma]$, the time being replaced by the coordinate $x_{3}[\sigma]$, while $\phi(x)$ contained in $y_{f}[\sigma]$ plays the role of another field interacting with the wave field. Equation (7.1c) gives the $\boldsymbol{\phi}(x)$ in terms of the canonical conjugate variables $\phi(x)$ and $\mu(x)$, and (7.1b) may be interpreted as the vacuum condition for the $\phi$-field (the corresponding condition for the wave field will be described in the next section). Equation (7.1a) will, of course, correspond to the probability amplitude function in the vacuum state of the $\phi$-field. On the other hand, the major difference from the field theory will be the nonexistence of the equation of motion for the medium variables $\phi, \mu$, and $\phi$ given in the form of $(7.1 \mathrm{~d})$, which would determine their behavior along the coordinate $x_{3}$.

In the case of quantum electrodynamics, the longitudinal field can be removed (on account of the Lorentz condition) to give rise to the Coulomb interaction terms between the charges involved in the Hamiltonian. ${ }^{13}$ Therefore, it will be interesting to check a few results that would come from the corresponding use of the Coulomb gauge in electrodynamics. On referring to (7.1a) or (5.51a), we observe from (6.3) that

$$
\begin{gathered}
i\left(\partial / \partial x_{3}\left[\sigma_{1}\right]\right)\left\langle\psi\left[\sigma_{1}\right]^{\prime}, \sigma_{1} \mid \psi\left[\sigma_{2}\right]^{\prime}, \sigma_{2}\right\rangle=\left\langle\psi\left[\sigma_{1}\right]^{\prime}, \sigma_{1}\left|\partial \mathcal{f}\left[\sigma_{1}\right]\right| \psi\left[\sigma_{2}\right]^{\prime}, \sigma_{2}\right\rangle, \\
-i\left(\partial / \partial x_{3}\left[\sigma_{2}\right]\right)\left\langle\psi\left[\sigma_{1}\right]^{\prime}, \sigma_{1} \mid \psi\left[\sigma_{2}\right]^{\prime}, \sigma_{2}\right\rangle=\left\langle\psi\left[\sigma_{1}\right]^{\prime}, \sigma_{1}\left|y f\left[\sigma_{2}\right]\right| \psi\left[\sigma_{2}\right]^{\prime}, \sigma_{2}\right\rangle,
\end{gathered}
$$

where, according to (7.2),

$$
\mathscr{H}[\sigma]=\mathscr{H}_{0}[\sigma]+\mathscr{H}_{1}[\sigma], \quad \mathscr{H}_{1}[\sigma]=\int_{\sigma} d \sigma_{3} j(x) \phi(x),
$$

when $\eta=\bar{\eta}=\eta^{*}=\bar{\eta}^{*}=0$.

On the other hand, according to the lemma (5.38),

$$
\left\langle\psi\left[\sigma_{1}\right]^{\prime}, \sigma_{1}|j(x) \phi(x)| \psi\left[\sigma_{2}\right]^{\prime}, \sigma_{2}\right\rangle=\left\{\langle\phi(x)\rangle_{0}+i(\delta / \delta q(x))\right\}\left\langle\psi\left[\sigma_{1}\right]^{\prime}, \sigma_{1}|j(x)| \psi\left[\sigma_{2}\right]^{\prime}, \sigma_{2}\right\rangle,
$$

where, according to $(5.35 \mathrm{a})$,

$$
\langle\phi(x)\rangle_{0}=-i \int_{\Sigma} D\left(x-x^{\prime}\right) q\left(x^{\prime}\right)\left(d x^{\prime}\right)
$$

In order to evaluate the functional derivative with respect to $q(x)$ in (7.5), we notice the lemma (6.29) with (6.30) and thus have

$$
i(\delta / \delta q(x))\left\langle\psi\left[\sigma_{1}\right]^{\prime}, \sigma_{1}|j(x)| \psi\left[\sigma_{2}\right]^{\prime}, \sigma_{2}\right\rangle=\left\langle\psi\left[\sigma_{1}\right]^{\prime}, \sigma_{1}\left|T\left[j(x) \int_{\sigma_{2}}^{\sigma_{1}} j\left(x^{\prime}\right)\left\{\delta \phi\left(x^{\prime}\right) / \delta q(x)\right\}\left(d x^{\prime}\right)\right]\right| \psi\left[\sigma_{2}\right]^{\prime}, \sigma_{2}\right\rangle
$$

where, according to $(7.1 \mathrm{c})$,

$$
\delta \phi\left(x^{\prime}\right) / \delta q(x)=-i D\left(x^{\prime}-x\right)
$$

${ }^{13}$ Coulomb potential is, of course, an ordinary potential not quantized. 
Therefore, we finally observe from (7.4), (7.5), (7.7) and (7.8) that, for $q=0$,

$\left\langle\psi\left[\sigma_{1}\right]^{\prime}, \sigma_{1}\left|\mathscr{H}_{1}\left[\sigma_{1}\right]\right| \psi\left[\sigma_{2}\right]^{\prime}, \sigma_{2}\right\rangle$

$$
=-i\left\langle\psi\left[\sigma_{1}\right]^{\prime}, \sigma_{1}\left|T\left[\int_{\sigma_{1}} d \sigma_{3}(x) \int_{\sigma_{2}}^{\sigma_{1}}\left(d x^{\prime}\right) j(x) D\left(x-x^{\prime}\right) j\left(x^{\prime}\right)\right]\right| \psi\left[\sigma_{2}\right]^{\prime}, \sigma_{2}\right\rangle, \quad q=0
$$

On the right side of (7.9), the $\phi$ variable is not present, and the term $-i j(x) D\left(x-x^{\prime}\right) j\left(x^{\prime}\right)$ may be interpreted as a two-body interaction between the "charges" $j(x)$ and $j(x$ ') with the interaction potential $-i D\left(x-x^{\prime}\right)$. However, a large difference from the electrodynamics is that it is an interaction of the charges on the surface $\sigma_{1}$ with all the charges distributed over the space between $\sigma_{1}$ and $\sigma_{2}$, and consequently, it follows that $\mathscr{P}\left[\sigma_{1}\right]$ in (7.3) depends not only on the wave variables on $\sigma_{1}$ but also on the variables on other surfaces involved between $\sigma_{1}$ and $\sigma_{2}$. It implies that the relation corresponding to (6.16) does not hold, and thus a new mathematical problem arises when trying to use (7.9).

\section{Expectation Values of Physical Variables and Their Relations With Green's Functions}

As mentioned in section 2 , the medium fluctuation is assumed to vanish adiabatically at infinity, and also the external sources $\eta=\bar{\eta}=\eta^{*}=\bar{\eta}^{*}=0$ there. Hence, we can explicitly define the incoming waves and the outgoing waves there such that

$$
\psi[\sigma]=\psi^{+}[\sigma]+\psi^{-}[\sigma], \quad \bar{\psi}[\sigma]=\bar{\psi}^{+}[\sigma]+\bar{\psi}^{-}[\sigma], \quad(d \psi[\sigma])=\left(d \psi^{+}[\sigma]\right)\left(d \psi^{-}[\sigma]\right),
$$

where $x_{3}[\sigma]= \pm \infty$, and $\psi^{ \pm}[\sigma]$ and $\bar{\psi}^{ \pm}[\sigma]$ are the components of $\psi[\sigma]$ and $\bar{\psi}[\sigma]$ belonging to the waves propagating in the positive and negative directions, respectively. According to (2.19), (8.1) is equivalent to

where

$$
\psi(x)=\psi^{+}(x)+\psi^{-}(x), \quad \bar{\psi}(x)=\bar{\psi}^{+}(x)+\bar{\psi}^{-}(x),
$$

$$
\psi^{ \pm}(x)=\psi_{a}(x) N_{a b} \psi_{b}^{ \pm}[\sigma], \quad \bar{\psi}^{ \pm}(x)=\bar{\psi}_{\bar{b}}^{ \pm}[\sigma] N_{b a} \psi_{a}^{*}(x)
$$

the explicit subscripts being attached.

When evaluating the expectation value of any physical variable, we notice that the excitation of the waves is given by the external current source, $\eta$ and/or by possible incoming waves prescribed at infinity. First, we shall consider $A v[A(x) B(y)]$ which is the average value of the product of two physical variables $A$ at $x$ and $B$ at $y$. Here the incoming wave components $\psi^{-}\left[\sigma_{1}\right]^{\prime}$ at $x_{3}\left[\sigma_{1}\right]=$ $+\infty$ and $\psi^{+}\left[\sigma_{2}\right]^{\prime}$ at $x_{3}\left[\sigma_{2}\right]=-\infty$ are assumed to be given, and the outgoing waves may have various possible values due to the fluctuation of the medium. Then, tracing the same course of evaluation as we did when deriving $(5.51 \mathrm{la})$, we have, if the point $x$ is on the surface $\sigma_{x}$ and the point $y$ on $\sigma_{y}$ and if $x_{3}\left[\sigma_{x}\right] \geqslant x_{3}\left[\sigma_{y}\right]$,

$\operatorname{Av}[A(x) B(y)]=\lim x_{3}\left[\sigma_{1}\right] \rightarrow+\infty, x_{3}\left[\sigma_{2}\right] \rightarrow-\infty$

$$
\times \frac{\iiint \iiint\left\langle 0_{\phi} \mid \phi[\Sigma]^{\prime}\right\rangle d \phi[\Sigma]^{\prime}\left(d \psi^{+}\left[\sigma_{1}\right]^{\prime}\right)\left\langle\zeta\left[\sigma_{1}\right]^{\prime}, \sigma_{1} \mid \zeta\left[\sigma_{x}\right]^{\prime}, \sigma_{x}\right\rangle A(x)^{\prime}\left(d \zeta\left[\sigma_{x}\right]^{\prime}\right)}{\iiint \int\left\langle 0_{\phi} \mid \phi[\Sigma]^{\prime}\right\rangle d \phi[\Sigma]^{\prime}\left(d \psi^{+}\left[\sigma_{1}\right]^{\prime}\right)\left\langle\zeta\left[\sigma_{1}\right]^{\prime}, \sigma_{1} \mid \zeta\left[\sigma_{2}\right]^{\prime}, \sigma_{2}\right\rangle}
$$

$* \frac{\left\langle\zeta\left[\sigma_{x}\right]^{\prime}, \sigma_{x} \mid \zeta\left[\sigma_{y}\right]^{\prime}, \sigma_{y}\right\rangle B(y)^{\prime}\left(d \zeta\left[\sigma_{y}\right]^{\prime}\right)\left\langle\zeta\left[\sigma_{y}\right]^{\prime}, \sigma_{y} \mid \zeta\left[\sigma_{2}\right]^{\prime}, \sigma_{2}\right\rangle\left(d \psi^{-}\left[\sigma_{2}\right]^{\prime}\right) d \phi[\Sigma]^{\prime \prime}\left\langle\phi[\Sigma]^{\prime \prime} \mid 0_{\mu}\right\rangle}{\left(d \psi^{-}\left[\sigma_{2}\right]^{\prime}\right) d \phi[\Sigma]^{\prime \prime}\left\langle\phi[\Sigma]^{\prime \prime} \mid 0_{\mu}\right\rangle}$

where

$$
\zeta\left[\sigma_{1}\right]^{\prime}=\left(\psi^{+}\left[\sigma_{1}\right]^{\prime}, \psi^{-}\left[\sigma_{1}\right]^{\prime}, \phi[\Sigma]^{\prime}\right), \quad \zeta\left[\sigma_{2}\right]^{\prime}=\left(\psi^{+}\left[\sigma_{2}\right]^{\prime}, \psi^{-}\left[\sigma_{2}\right]^{\prime}, \phi[\Sigma]^{\prime \prime}\right)
$$


Here, it is very convenient to introduce the right- and left-hand Hilbert vectors $\left|0^{+}\right\rangle$and $\left\langle 0^{-}\right|$ defined by

$$
\begin{aligned}
& \psi^{+}\left[\sigma_{2}\right]\left|0^{+}\right\rangle=\bar{\psi}^{+}\left[\sigma_{2}\right]\left|0^{+}\right\rangle=\psi^{+*}\left[\sigma_{2}\right]\left|0^{+}\right\rangle=\bar{\psi}^{+*}\left[\sigma_{2}\right]\left|0^{+}\right\rangle=0, \\
& \left\langle 0^{-}\right| \psi^{-}\left[\sigma_{1}\right]=\left\langle 0^{-}\right| \bar{\psi}^{-}\left[\sigma_{1}\right]=\left\langle 0^{-}\right| \psi^{-*}\left[\sigma_{1}\right]=\left\langle 0^{-}\right| \bar{\psi}^{-*}\left[\sigma_{1}\right]=0,
\end{aligned}
$$

or, in the representation in which $\psi^{+}[\sigma]$ and $\bar{\psi}^{+}[\sigma]$ or $\psi^{-}[\sigma]$ and $\bar{\psi}^{-}[\sigma]$ are simultaneously diagonal, their matrix elements may be given by

$$
\begin{aligned}
& \left\langle\psi^{+}\left[\sigma_{2}\right]^{\prime}, \bar{\psi}^{+}\left[\sigma_{2}\right]^{\prime} \mid 0^{+}\right\rangle=\delta\left(\psi^{+}\left[\sigma_{2}\right]^{\prime}\right) \delta\left(\bar{\psi}^{+}\left[\sigma_{2}\right]^{\prime} / 2 \pi\right), \\
& \left\langle 0^{-} \mid \psi^{-}\left[\sigma_{1}\right]^{\prime}, \bar{\psi}^{-}\left[\sigma_{1}\right]^{\prime}\right\rangle=\delta\left(\psi^{-}\left[\sigma_{1}\right]^{\prime}\right) \delta\left(\bar{\psi}^{-}\left[\sigma_{1}\right]^{\prime} / 2 \pi\right),
\end{aligned}
$$

where the $\delta$-function in this case is defined as in (5.2). The above representation is possible since $\psi^{ \pm}[\sigma]$ and $\bar{\psi}^{ \pm}[\sigma]$ commute each other and thus they can be represented simultaneously in diagonal; using the orthogonality (2.39) and the commutation relation $(6.9 \mathrm{a}-\mathrm{c})$,

$$
\begin{aligned}
& {\left[\bar{\psi}_{a}^{ \pm}[\sigma], \psi_{b}^{ \pm}[\sigma]\right]=\left[\bar{\psi}_{a}^{ \pm *}[\sigma], \psi_{b}^{ \pm *}[\sigma]\right]=0,} \\
& {\left[\frac{1}{2}\left\{\bar{\psi}^{ \pm}[\sigma] N\right\}_{a}, \psi_{b}[\sigma]\right]=-i \delta_{a b} .}
\end{aligned}
$$

In order to get the representations of $\left|0^{+}\right\rangle$and $\left\langle 0^{-}\right|$in which $\psi\left[\sigma_{2}\right]$ and $\psi\left[\sigma_{1}\right]$ are diagonal, respectively, the transformation function $\left\langle\psi[\sigma]^{\prime} \mid \bar{\psi}[\sigma]^{\prime}\right\rangle$ between the two variables $\psi[\sigma]$ and $\bar{\psi}[\sigma]$ is necessary and is given by

$$
\begin{aligned}
&\left\langle\psi[\sigma]^{\prime} \mid \bar{\psi}[\sigma]^{\prime}\right\rangle=\exp \left[i \frac{1}{2} \int_{\sigma} d \sigma_{3}\left\{\bar{\psi}^{\prime} \gamma_{3} \psi^{\prime}+\psi^{* \prime} \gamma_{3} \bar{\psi}^{* \prime}\right\}\right] \\
&=\exp \left[i \frac{1}{2}\left\{\bar{\psi} a[\sigma]^{\prime} N_{a b} \psi_{b}[\sigma]^{\prime}+\psi_{b}^{*}[\sigma]^{\prime} N_{b a} \bar{\psi}_{a}^{*}[\sigma]^{\prime}\right\}\right] \\
&=\left\langle\psi^{+}[\sigma]^{\prime} \mid \bar{\psi}^{-}[\sigma]^{\prime}\right\rangle\left\langle\psi^{-}[\sigma]^{\prime} \mid \bar{\psi}^{+}[\sigma]^{\prime}\right\rangle, \\
&\left\langle\psi^{ \pm}[\sigma]^{\prime} \mid \bar{\psi}^{\mp}[\sigma]^{\prime}\right\rangle=\exp \left[i \frac{1}{2}\left\{\bar{\psi}_{a}^{\mp}[\sigma]^{\prime} N_{a b} \psi_{b}[\sigma]^{\prime}+\psi_{b}^{+*}[\sigma]^{\prime} N_{b a} \bar{\psi}_{a}^{\mp *}[\sigma]^{\prime}\right\}\right],
\end{aligned}
$$

where, by the use of (6.5), it is easy to see that

$$
\int\left\langle\psi[\sigma]^{\prime}\left|\frac{1}{2}\{\bar{\psi}[\sigma] N\}_{a}\right| \psi[\sigma]^{\prime \prime}\right\rangle\left(d \psi[\sigma]^{\prime \prime}\right)\left\langle\psi[\sigma]^{\prime \prime} \mid \bar{\psi}[\sigma]^{\prime}\right\rangle=\frac{1}{2}\left\{\bar{\psi}[\sigma]^{\prime} N\right\}_{a}\left\langle\psi[\sigma]^{\prime} \mid \bar{\psi}[\sigma]^{\prime}\right\rangle,
$$

and a similar equation holds for $\frac{1}{2}\left\{N \bar{\psi}^{*}[\sigma]\right\}_{a}$. Also, it is normalized in such a way that

$$
\int\left\langle\psi[\sigma]^{\prime} \mid \bar{\psi}[\sigma]^{\prime}\right\rangle\left(d \bar{\psi}[\sigma]^{\prime} / 2 \pi\right)\left\langle\bar{\psi}[\sigma]^{\prime} \mid \psi[\sigma]^{\prime \prime}\right\rangle=\delta\left(\psi[\sigma]^{\prime}-\psi[\sigma]^{\prime \prime}\right) .
$$

Thus, using (8.8),

$$
\begin{aligned}
& \left\langle\psi\left[\sigma_{2}\right]^{\prime} \mid 0^{+}\right\rangle \equiv\left\langle\psi^{+}\left[\sigma_{2}\right]^{\prime}, \psi^{-}\left[\sigma_{2}\right]^{\prime} \mid 0^{+}\right\rangle=\int\left\langle\psi^{-}\left[\sigma_{2}\right]^{\prime} \mid \bar{\psi}^{+}\left[\sigma_{2}\right]^{\prime}\right\rangle \\
& \quad \times\left(d \bar{\psi}^{+}\left[\sigma_{2}\right]^{\prime} / 2 \pi\right)\left\langle\psi^{+}\left[\sigma_{2}\right]^{\prime}, \bar{\psi}^{+}\left[\sigma_{2}\right]^{\prime} \mid 0^{+}\right\rangle=\delta\left(\psi^{+}\left[\sigma_{2}\right]^{\prime}\right) .
\end{aligned}
$$

In the same way,

$$
\left\langle 0^{-} \mid \psi\left[\sigma_{1}\right]^{\prime}\right\rangle=\delta\left(\psi^{-}\left[\sigma_{1}\right]^{\prime}\right)
$$


We observe from (8.11) that $\left|0^{+}\right\rangle$has a uniform distribution with respect to $\psi^{-}\left[\sigma_{2}\right]^{\prime}$ and $\left\langle 0^{-}\right|$ with respect to $\psi^{+}\left[\sigma_{1}\right]^{\prime}$. It implies that any eigenvector of the eigenvalue $\bar{\psi}^{+}[\sigma]^{\prime}=0$ represents the uniform distribution with respect to $\psi^{-}[\sigma]^{\prime}$, and any vector of $\bar{\psi}^{-}[\sigma]^{\prime}=0$ with respect to $\psi^{+}[\sigma]^{\prime}$.

Therefore, when there are no incoming waves at the infinity and thus $\psi^{-}\left[\sigma_{1}\right]^{\prime}=\psi^{+}\left[\sigma_{2}\right]^{\prime}=0$ in (8.4), the right side of (8.4) can be entirely expressed in terms of $\left\langle 0^{-}\right|$and $\left|0^{+}\right\rangle$as follows: using $(8.11 \mathrm{a}, \mathrm{b})$,

$$
\begin{aligned}
\iint\left\langle 0_{\phi} \mid \phi[\Sigma]^{\prime}\right\rangle & d \phi[\Sigma]^{\prime}\left(d \psi^{+}\left[\sigma_{1}\right]^{\prime}\right)\left\langle\zeta\left[\sigma_{1}\right]^{\prime}, \sigma_{1} \mid \zeta[\sigma]^{\prime}, \sigma\right\rangle \\
& =\int\left\langle 0_{\phi} \mid \phi[\Sigma]^{\prime}\right\rangle\left\langle 0^{-} \mid \psi\left[\sigma_{1}\right]^{\prime}\right\rangle\left(d \zeta\left[\sigma_{1}\right]^{\prime}\right)\left\langle\zeta\left[\sigma_{1}\right]^{\prime}, \sigma_{1} \mid \zeta[\sigma]^{\prime}, \sigma\right\rangle \equiv\left\langle 0_{\phi}\right|\left\langle 0^{-} \mid \zeta[\sigma]^{\prime}, \sigma\right\rangle,
\end{aligned}
$$

$\iint\left\langle\zeta[\sigma]^{\prime}, \sigma \mid \zeta\left[\sigma_{2}\right]^{\prime}, \sigma_{2}\right\rangle\left(d \psi^{-}\left[\sigma_{2}\right]^{\prime}\right) d \phi[\Sigma]^{\prime}\left\langle\phi[\Sigma]^{\prime} \mid 0_{\mu}\right\rangle=\left\langle\zeta[\sigma]^{\prime}, \sigma \mid 0^{+}\right\rangle\left|0_{\mu}\right\rangle$.

Thus, in the case of no incoming waves, (8.4) can be given by

$$
\mathrm{A} v[A(x) B(y)]=\langle T[A(x) B(y)]\rangle, \quad \psi^{-}\left[\sigma_{1}\right]^{\prime}=\psi^{+}\left[\sigma_{2}\right]^{\prime}=0,
$$

in terms of the notations

with

$$
\langle F\rangle \equiv\langle 0|F| 0\rangle /\langle 0 \mid 0\rangle,
$$

$$
\left\langle 0\left|\equiv\left\langle 0_{\phi}\left|\left\langle 0^{-}|, \quad| 0\right\rangle \equiv\right| 0^{+}\right\rangle\right| 0_{\mu}\right\rangle ;
$$

this new notation is to be distinguished from the similar one $\langle F\rangle_{0}$ introduced in (5.35b).

$\langle F\rangle$ is generally a functional of the external sources $\eta, \eta^{*}, \bar{\eta}, \bar{\eta}^{*}$, and $q$, and it will usually be possible to expand it into the power series, e.g.,

$$
\begin{aligned}
\langle F\rangle & =\left.\sum_{n=0}^{\infty} \frac{1}{n !}\left[\int_{\Sigma}(d x)\left\{\eta_{\alpha}(x)^{\prime}\left(\delta / \delta \eta_{\alpha}(x)\right)+\eta_{\alpha}^{*}(x)^{\prime}\left(\delta / \delta \eta_{\alpha}^{*}(x)\right)\right\}\right]^{n}\langle F\rangle\right|_{\eta=\eta^{*}=0} \\
& =\left.\exp \left[\int_{\Sigma}(d x)\left\{\eta_{\alpha}(x)^{\prime}\left(\delta / \delta \eta_{\alpha}(x)\right)+\eta_{\alpha}^{*}(x)^{\prime}\left(\delta / \delta \eta_{\alpha}^{*}(x)\right)\right\}\right]\langle F\rangle\right|_{\eta=\eta^{*}=0}
\end{aligned}
$$

which is the expansion with respect to $\eta=\eta^{\prime}$ and $\eta^{*}=\eta^{* \prime}$. The above series, however, terminates after a finite number of term in the case where $F$ is a polynomial of the wave variables $\psi$ and $\psi^{*}$, being the case of most interest, as will be seen later (Green's functions).

On the other hand, from (6.26) and (6.28), we see, if $T[F]=F$, which does not contain $\eta$ and $\eta^{*}$ explicitly, that

$$
\begin{aligned}
& \left(\delta / \delta \eta_{\alpha}(x)\right)^{n}\langle 0|F| 0\rangle=\left\langle 0\left|T\left[F\left\{-\frac{1}{2} \bar{\psi}_{\alpha}(x)\right\}^{n}\right]\right| 0\right\rangle, \\
& \left(\delta / \delta \eta_{\alpha}^{*}(x)\right)^{n}\langle 0|F| 0\rangle=\left\langle 0\left|T\left[F\left\{\frac{1}{2} \bar{\psi}_{\alpha}^{*}(x)\right\}^{n}\right]\right| 0\right\rangle .
\end{aligned}
$$

Therefore, on referring to (8.15), we have, if $T[F]=F$,

with

$$
\left.\langle 0|F| 0\rangle\right|_{\eta=\eta^{\prime}, \eta^{*}=\eta^{*}}=\left.\langle 0|T[\exp (-i w) F]| 0\rangle\right|_{\eta=\eta^{*}=0},
$$

$$
w=\frac{i}{2} \int_{\Sigma}\left\{-\bar{\psi}(x) \eta(x)^{\prime}+\eta^{*}(x)^{\prime} \bar{\psi}^{*}(x)\right\}(d x) .
$$

Thus, using (8.17) and (8.18), we observe from (8.13), dropping the primes from $\eta^{\prime}$ and $\eta^{* \prime}$, that

$$
\operatorname{Av}[F]=\langle T[F]\rangle=\langle 0|T[\exp (-i w) F]| 0\rangle /\langle 0|T[\exp (-i w)]| 0\rangle,
$$

where $\eta=\eta^{*}=0$ on the right side except for those in the'term $w$. 
In the same way, we can get the similar explicit dependence on $\bar{\eta}$ and $\bar{\eta}^{*}$ by the use of (6.29) with (6.30).

The equation (8.19) gives the explicit dependence of the external sources on the expectation value of any physical variable involved when there are no incoming waves at the infinity. Here it is remarked that the same equation (8.19) can be used even when the excitation of the system is provided by incoming waves $\psi^{-}\left[\sigma_{1}\right]^{\prime}$ and $\psi^{+}\left[\sigma_{2}\right]^{\prime}$ prescribed at the infinities $x_{3}\left[\sigma_{1}\right]=+\infty$ and $x_{3}\left[\sigma_{2}\right]=-\infty$, respectively, by introducing the following effective values $\eta_{\psi}$ of $\eta$ and $\eta_{\psi}^{*}$ of $\eta^{*}$ :

$$
\begin{gathered}
\eta_{\psi}(x)=i \gamma_{3}\left[-\psi^{-}\left(\sigma_{1}(x)\right)^{\prime} \delta\left(x_{3}[\sigma]-x_{3}\left[\sigma_{1}\right]\right)+\psi^{+}\left(\sigma_{2}(x)\right)^{\prime} \delta\left(x_{3}[\sigma]-x_{3}\left[\sigma_{2}\right]\right)\right], \\
\eta_{\psi}^{*}(x)=i\left[\psi^{-*}\left(\sigma_{1}(x)\right)^{\prime} \delta\left(x_{3}[\sigma]-x_{3}\left[\sigma_{1}\right]\right)-\psi^{+*}\left(\sigma_{2}(x)\right)^{\prime} \delta\left(x_{3}[\sigma]-x_{3}\left[\sigma_{2}\right]\right)\right] \gamma_{3} .
\end{gathered}
$$

Here $\psi^{ \pm}(x)$ is defined by (8.3) and $\sigma$ is the surface on which the point $x$ exists. The proof of (8.20) is not difficult if one notes that $\left|\psi^{+}\left[\sigma_{2}\right]^{\prime}\right\rangle$ and $\left\langle\psi^{-}\left[\sigma_{1}\right]^{\prime}\right|$ defined by

$$
\begin{aligned}
\left|\psi^{+}\left[\sigma_{2}\right]^{\prime}\right\rangle & \equiv \exp \left[-\frac{i}{2} \int_{\sigma_{2}} d \sigma_{3}\left\{\bar{\psi} \gamma_{3} \psi^{+\prime}+\psi^{+* \prime} \gamma_{3} \bar{\psi}^{*}\right\}\right]\left|0^{+}\right\rangle, \\
\left\langle\psi^{-}\left[\sigma_{1}\right]^{\prime}\right| & \equiv\left\langle 0^{-}\right| \exp \left[\frac{i}{2} \int_{\sigma_{1}} d \sigma_{3}\left\{\bar{\psi} \gamma_{3} \psi^{-\prime}+\psi^{-* \prime} \gamma_{3} \bar{\psi}^{*}\right\}\right],
\end{aligned}
$$

turn out to be the eigenvectors of the eigenvalues $\psi^{+}\left[\sigma_{2}\right]^{\prime}$ and $\psi^{-}\left[\sigma_{1}\right]^{\prime}$, respectively (appendix 5).

In the same way as in (8.19), we could get the explicit dependence of $q$ on $\langle F\rangle$ according to (5.45). Thus, in the most general case where all the external sources $\eta, \eta^{*}, \bar{\eta}, \bar{\eta}^{*}$, and $q$ are not zero, we find, on referring to (8.17) and (8.18), that the equation (8.19) still gives their explicit dependence, if $w$ in (8.18) is replaced by

$$
w=\int_{\Sigma}(d x)\left[\phi(x) q(x)+\frac{i}{2}\left\{-\bar{\psi}(x) \eta(x)-\bar{\eta}(x) \psi(x)+\eta^{*}(x) \bar{\psi}^{*}(x)+\psi^{*}(x) \bar{\eta}^{*}(x)\right\}\right] .
$$

As a few direct results of (8.19) with (8.22), we have, for $T[F]=F$ which does not contain $\eta$ and $\eta^{*}$ explicitly,

$$
\begin{aligned}
& \left(\delta / \delta \eta_{\alpha}(x)\right)\langle F\rangle=-\frac{1}{2}\left\langle T\left[F \bar{\psi}_{\alpha}(x)\right]\right\rangle+\frac{1}{2}\langle F\rangle\left\langle\bar{\psi}_{\alpha}(x)\right\rangle, \\
& \left(\delta / \delta \eta_{\alpha}^{*}(x)\right)\langle F\rangle=\frac{1}{2}\left\langle T\left[F \bar{\psi}_{\alpha}^{*}(x)\right]\right\rangle-\frac{1}{2}\langle F\rangle\left\langle\bar{\psi}_{\alpha}^{*}(x)\right\rangle, \\
& i(\delta / \delta q(x))\langle F\rangle=\langle T[F \phi(x)]\rangle-\langle F\rangle\langle\phi(x)\rangle,
\end{aligned}
$$

and also the similar equations with respect to $\bar{\eta}_{\alpha}(x)$ and $\bar{\eta}_{\alpha}{ }^{*}(x)$.

On the other hand, in the same way as (7.5) is obtained, we have

$$
\langle\phi(x)\rangle=\langle\phi(x)\rangle_{0}+i(\delta / \delta q(x))\langle 0 \mid 0\rangle /\langle 0 \mid 0\rangle
$$

while, tracing the same course of evaluation as in (7.7) with (7.8),

Hence,

$$
i(\delta / \delta q(x))\langle 0 \mid 0\rangle=-i \int_{\Sigma} D\left(x-x^{\prime}\right)\left\langle 0\left|j\left(x^{\prime}\right)\right| 0\right\rangle\left(d x^{\prime}\right)
$$

$$
\begin{aligned}
\langle\phi(x)\rangle & =\langle\phi(x)\rangle_{0}-i \int_{\Sigma} D\left(x-x^{\prime}\right)\left\langle j\left(x^{\prime}\right)\right\rangle\left(d x^{\prime}\right) \\
& =-i \int_{\Sigma} D\left(x-x^{\prime}\right)\left\{q\left(x^{\prime}\right)+\left\langle j\left(x^{\prime}\right)\right\rangle\right\}\left(d x^{\prime}\right) .
\end{aligned}
$$
field.

${ }^{14}$ Using the field theoretical statement, $\langle j(x)\rangle$ corresponds to the vacuum fluctuation current, e.g., of the electron 
However, when the nonphysical external sources $\bar{\eta}=\bar{\eta}^{*}=0,\langle\boldsymbol{\phi}(x)\rangle$ should be independent of the wave variables since it is the expectation value of the fluctuating part of the medium, and thus $\langle\phi(x)\rangle=\langle\phi(x)\rangle_{0}$ or $\langle j(x)\rangle=0$.

The equation for $\langle\psi(x)\rangle$, corresponding to (8.25), is obtained by applying the brackets $\langle$ and $\rangle$ to the left and right sides of $(6.23 \mathrm{~b})$ with (5.8) to give

$$
\left[(\gamma \partial)-k_{0}\right]\langle\psi(x)\rangle-\gamma\langle\phi(x) \psi(x)\rangle=\eta(x),
$$

which, using the lemma (8.23c) for the second term on the left side, gives

$$
\left[(\gamma \partial)-k_{0}-\gamma\langle\phi(x)\rangle-i \gamma(\delta / \delta q(x))\right]\langle\psi(x)\rangle=\eta(x) .
$$

Here, according to the boundary condition $(8.5),\left\langle\psi^{ \pm}(x)\right\rangle$ are to vanish at $x_{3}=\mp \infty$, respectively, and thus $\langle\psi(x)\rangle$ satisfies the outward propagating wave condition at infinity.

In the same way, for $\langle\bar{\psi}(x)\rangle$, we have

$$
\langle\bar{\psi}(x)\rangle\left[-(\gamma \overleftarrow{\partial})-k_{0}-\gamma\langle\phi(x)\rangle-i \gamma(\overleftarrow{\delta / \delta q(x)})\right]=\bar{\eta}(x)
$$

and also the similar equations for $\left\langle\psi^{*}(x)\right\rangle$ and $\left\langle\bar{\psi}^{*}(x)\right\rangle$.

The Green's functions $G_{\alpha \beta}\left(x, x^{\prime}\right)$ and $G_{\alpha \beta}^{*}\left(x, x^{\prime}\right)$ for the original wave function and the complex conjugate wave function are respectively defined, on referring to (8.23) and also (6.32), by

$$
\begin{aligned}
G_{\alpha \beta}\left(x, x^{\prime}\right) & =\left(\delta / \delta \eta_{\beta}\left(x^{\prime}\right)\right)\left\langle\psi_{\alpha}(x)\right\rangle \\
& =-\frac{1}{2}\left\langle T\left[\psi_{\alpha}(x) \bar{\psi}_{\beta}\left(x^{\prime}\right)\right]\right\rangle-\left\{\left\langle k_{33}(x)^{-1}\right\rangle\left(1-\gamma_{3}^{2}\right)\right\}_{\alpha \beta} \delta\left(x-x^{\prime}\right), \\
G_{\alpha \beta}^{*}\left(x, x^{\prime}\right) & =\left(\delta / \delta \eta_{\beta}^{*}\left(x^{\prime}\right)\right)\left\langle\psi_{\alpha}^{*}(x)\right\rangle \\
& =\frac{1}{2}\left\langle T\left[\psi_{\alpha}^{*}(x) \bar{\psi}_{\beta}^{*}\left(x^{\prime}\right)\right]\right\rangle-\left\{\left\langle k_{33}(x)^{-1}\right\rangle\left(1-\gamma_{3}^{2}\right)\right\}_{\alpha \beta} \delta\left(x-x^{\prime}\right),
\end{aligned}
$$

where $\eta, \eta^{*}, \bar{\eta}$, and $\bar{\eta}^{*}$ are to vanish after the functional differentiations (but $q \neq 0$ for a while). Also $\langle\psi(x)\rangle=\left\langle\psi^{*}(x)\right\rangle=0$ for $\eta=\eta^{*}=0$, according to (8.26). In the same way, we can define the Green's function $g\left(x, x^{\prime}\right)$ of the medium by

$$
\begin{aligned}
g\left(x, x^{\prime}\right)=i\left(\delta / \delta q\left(x^{\prime}\right)\right)\langle\phi(x)\rangle=\left\langle T\left[\phi(x) \phi\left(x^{\prime}\right)\right]\right\rangle-\langle\phi(x)\rangle\left\langle\phi\left(x^{\prime}\right)\right\rangle & \\
& =D\left(x-x^{\prime}\right)+\int_{\Sigma}\left(d x^{\prime \prime}\right) D\left(x-x^{\prime \prime}\right)\left(\delta / \delta q\left(x^{\prime}\right)\right)\left\langle j\left(x^{\prime \prime}\right)\right\rangle=D\left(x-x^{\prime}\right),
\end{aligned}
$$

where $(8.23 \mathrm{c})$ and $(8.25)$ are used and $\eta=\eta^{*}=\bar{\eta}=\bar{\eta}^{*}=0$.

On the other hand, operating $\delta / \delta \eta(x)$ on both sides of $(8.26 \mathrm{a})$, and putting $\eta=\eta^{*}=\bar{\eta}=\bar{\eta}^{*}=0$ after it, we have, according to the definition (8.27) of the Green's functions,

$$
\left[(\gamma \partial)-k_{0}-\gamma\langle\phi(x)\rangle-i \gamma(\delta / \delta q(x))\right] G\left(x, x^{\prime}\right)=\delta\left(x-x^{\prime}\right)
$$

The equation (8.29) is also derived directly from the explicit expression in (8.27) on using the wave equation $(6.23 \mathrm{~b})$ for $\eta=0$ and the commutation relation $(6.9 \mathrm{a}-\mathrm{c})$.

In the same way,

$$
\left[(\gamma \partial)-k_{0}^{*}-\gamma^{*}\langle\phi(x)\rangle-i \gamma^{*}(\delta / \delta q(x))\right] G^{*}\left(x, x^{\prime}\right)=\delta\left(x-x^{\prime}\right)
$$


Here, $\langle\phi(x)\rangle$ in (8.29) and (8.30) is given by (8.25) with $\langle j(x)\rangle=0 . .^{15}$ Thus, the simultaneous equations (8.29) and (8.30) with (8.25) give a rigorous description of $G\left(x, x^{\prime}\right)$ and $G^{*}\left(x, x^{\prime}\right)$.

In a similar way, we may define a double Green's function by

$$
G\left(x_{1} ; x_{2} \mid x_{1}^{\prime} ; x_{2}^{\prime}\right)=\left.\left(\delta / \delta \eta\left(x_{1}^{\prime}\right)\right)\left(\delta / \delta \eta^{*}\left(x_{2}^{\prime}\right)\right)\left\langle T\left[\psi\left(x_{1}\right) \psi^{*}\left(x_{2}\right)\right]\right\rangle\right|_{\eta=\eta^{*}=\bar{\eta}=\bar{\eta}^{*}=0}
$$

where the subscripts for the wave components are included in the coordinates $x_{i}$ and $x_{i}^{\prime}$. Using (8.27), this function is given by

$$
\begin{aligned}
& G\left(x_{1} ; x_{2} \mid x_{1}^{\prime} ; x_{2}^{\prime}\right)=-\frac{1}{4}\left\langle T\left[\psi\left(x_{1}\right) \psi^{*}\left(x_{2}\right) \bar{\psi}\left(x_{1}^{\prime}\right) \bar{\psi}^{*}\left(x_{2}^{\prime}\right)\right]\right\rangle \\
& -\left(\delta / \delta \eta^{*}\left(x_{2}^{\prime}\right)\right)\left\langle\psi^{*}\left(x_{2}\right) k_{33}\left(x_{1}\right)^{-1}\right\rangle\left(1-\gamma_{3}^{2}\right)_{1} \delta\left(x_{1}-x_{1}^{\prime}\right)-\left(\delta / \delta \eta\left(x_{1}^{\prime}\right)\right)\left\langle\psi\left(x_{1}\right) k_{33}\left(x_{2}\right)^{-1}\right\rangle\left(1-\gamma_{3}^{2}\right)_{2} \delta\left(x_{2}-x_{2}^{\prime}\right) \\
& -\left\langle k_{33}\left(x_{1}\right)^{-1} k_{33}\left(x_{2}\right)^{-1}\right\rangle\left(1-\gamma_{3}^{2}\right)_{1}\left(1-\gamma_{3}^{2}\right)_{2} \delta\left(x_{1}-x_{1}^{\prime}\right) \delta\left(x_{2}-x_{2}^{\prime}\right)
\end{aligned}
$$

where $\left(1-\gamma_{3}^{2}\right)$ i, $i=1,2$, operate on the coordinates $x_{i}$.

On the other hand, in the same way as $(8.26 \mathrm{a})$ is derived,

$$
\mathscr{F}\left(x_{1}\right)\left\langle T\left[\psi\left(x_{1}\right) \psi^{*}\left(x_{2}\right)\right]\right\rangle=\eta\left(x_{1}\right)\left\langle\psi^{*}\left(x_{2}\right)\right\rangle
$$

where $\mathscr{F}(x)$ is the functional differential operator on the left side of (8.26a) or (8.29). The above equation further reads, using the complex conjugate of (8.26a),

$$
\mathscr{F} *\left(x_{2}\right) \mathscr{F}\left(x_{1}\right)\left\langle T\left[\psi\left(x_{1}\right) \psi^{*}\left(x_{2}\right)\right]\right\rangle=\eta\left(x_{1}\right) \eta^{*}\left(x_{2}\right),
$$

where $\mathscr{F}^{*}(x)$ is the functional operator on the left side of $(8.30)$.

Applying $\delta / \delta \eta\left(x_{1}^{\prime}\right)$ and $\delta / \delta \eta^{*}\left(x_{2}^{\prime}\right)$ on both sides of (8.33) and putting $\eta=\eta^{*}=\bar{\eta}=\bar{\eta}^{*}=0$ after it,

$$
\mathscr{F}^{*}\left(x_{2}\right) \mathscr{F}\left(x_{1}\right) G\left(x_{1} ; x_{2} \mid x_{1}^{\prime} ; x_{2}^{\prime}\right)=\delta\left(x_{1}-x_{1}^{\prime}\right) \delta\left(x_{2}-x_{2}^{\prime}\right)
$$

Here, the two differential operators $\mathscr{F}\left(x_{1}\right)$ and $\mathscr{F} *\left(x_{2}\right)$ are commutative on account of the symmetry $g\left(x, x^{\prime}\right)=g\left(x^{\prime}, x\right)$ from $(8.28)$.

Generally, the Green's functions of waves are defined by

$$
\begin{aligned}
G\left(x_{1}, x_{2}, \ldots . x_{n} ; y_{1}, y_{2}, \ldots ., y_{m} \mid x_{1}^{\prime}, x_{2}^{\prime}, \ldots, x_{n}^{\prime} ; y_{1}^{\prime}, y_{2}^{\prime}, \ldots ., y_{m}^{\prime}\right) \\
=\left\{\left(\delta / \delta \eta\left(x_{1}^{\prime}\right)\right)\left(\delta / \delta \eta\left(x_{2}^{\prime}\right)\right) \ldots .\left(\delta / \delta \eta\left(x_{n}^{\prime}\right)\right)\right\}\left\{\left(\delta / \delta \eta^{*}\left(y_{1}^{\prime}\right)\right)\left(\delta / \delta \eta^{*}\left(y_{2}^{\prime}\right)\right) \ldots .\left(\delta / \delta \eta^{*}\left(y_{m}^{\prime}\right)\right)\right\} \\
\quad \times\left.\left\langle\psi\left(x_{1}\right) \psi\left(x_{2}\right) \ldots \ldots\left(x_{n}\right) \psi^{*}\left(y_{1}\right) \psi^{*}\left(y_{2}\right) \ldots . \psi^{*}\left(y_{m}\right)\right\rangle\right|_{\eta=\eta^{*}=\bar{\eta}=\bar{\eta}^{*}=0},
\end{aligned}
$$

and all the other Green's functions which might be possible vanish identically; for instance,

$$
G\left(x_{1} \mid x_{2}, x_{3}\right)=\left.\left(\delta / \delta \eta\left(x_{2}\right)\right)\left(\delta / \delta \eta\left(x_{3}\right)\right)\left\langle\psi\left(x_{1}\right)\right\rangle\right|_{\eta=\eta^{*}=\bar{\eta}=\bar{\eta}^{*}=0}
$$

${ }^{15}$ According to the definitions (6.31) and (8.27) for $j(x)$ and the Green's functions, respectively,

$$
\begin{aligned}
\langle j(x)\rangle & =\left.i \operatorname{Tr}\left[\gamma G\left(x, x^{\prime}\right)+\gamma^{*} G^{*}\left(x, x^{\prime}\right)\right]\right|_{x^{\prime} \rightarrow x \pm 0} \\
& \left.=i \operatorname{Tr} \gamma\left\{G\left(x, x^{\prime}\right)+G^{+}\left(x^{\prime}, x\right)\right\}\right]\left.\right|_{x^{\prime} \rightarrow x \pm 0},
\end{aligned}
$$

where $G_{\dot{\beta} \alpha}^{+}\left(x^{\prime}, x\right)=G_{\alpha \beta}^{*}\left(x, x^{\prime}\right)$. Now it takes the same form as given for the corresponding vacuum current in field theory. See, for instance, Schwinger [1951]. 
should vanish because, otherwise, it would imply a quadratic dependence of $\left\langle\psi\left(x_{1}\right)\right\rangle$ on the external source $\eta$ according to the expansion (8.15).

According to (8.35), the expectation value of any functional $F$ of $\psi$ and $\psi^{*}$ proves to be expressible in terms of the Green's functions in view of the series expansion (8.15).

In the next section, the theory of the Green's functions will be developed, starting from (8.25), (8.29), and (8.30), by using the unperturbational method of Schwinger for quantum electrodynamics.

\section{Theory of Green's Functions of Waves}

The functional differential equation (8.29) is equivalent, as will be shown later, to the integrodifferential equation

$$
\left[(\gamma \partial)-k_{0}-\gamma\langle\boldsymbol{\phi}(x)\rangle\right] G\left(x, x^{\prime}\right)-\int \Delta k\left(x, x^{\prime \prime}\right)\left(d x^{\prime \prime}\right) G\left(x^{\prime \prime}, x^{\prime}\right)=\delta\left(x-x^{\prime}\right) .
$$

Here $\langle\boldsymbol{\phi}(x)\rangle$ is given by (8.25) with $\langle j(x)\rangle=0$ and the operator $\Delta k$ is defined later. According to the boundary conditions (8.5), $G\left(x, x^{\prime}\right)$ is to be the outward propagating wave at infinity, as stated below (8.26a).

Following Schwinger [1951], it is convenient to regard $G\left(x, x^{\prime}\right)$ and $\gamma$ as the following matrices with respect to the coordinates $x$ and $x^{\prime}$ including the subscripts:

$$
\left\langle x|G| x^{\prime}\right\rangle=G\left(x, x^{\prime}\right), \quad\left\langle x|\gamma(\xi)| x^{\prime}\right\rangle=\gamma \delta(x-\xi) \delta\left(x-x^{\prime}\right), \quad\left\langle x|p| x^{\prime}\right\rangle=i(\partial / \partial x) \delta\left(x-x^{\prime}\right) .
$$

Then, $\gamma\langle\phi(x)\rangle$ and $\gamma(\delta / \delta q(x))$ in (8.29) are represented by matrices as

$$
\gamma\langle\boldsymbol{\phi}(x)\rangle \rightarrow \int \gamma(\xi)\langle\boldsymbol{\phi}(\xi)\rangle(d \xi), \quad \gamma(\delta / \delta q(x)) \rightarrow \int \gamma(\xi)(\delta / \delta q(\xi))(d \xi),
$$

Thus, on comparing with (9.1), (8.29) can be given in the form

$$
\left[(\gamma p)-k_{0}-\int \gamma(\xi)\langle\boldsymbol{\phi}(\xi)\rangle(d \xi)-\Delta k\right] G=1,
$$

where the operator $\Delta k$ is defined by

$$
\Delta k G=i \int(d \xi) \gamma(\xi)(\delta / \delta q(\xi)) G
$$

In the same way, from (8.30),

$$
\left[(\gamma p)-k_{0}^{*}-\int \gamma^{*}(\xi)\langle\boldsymbol{\phi}(\xi)\rangle(d \xi)-\Delta k^{*}\right] G^{*}=1, \quad \Delta k^{*} G^{*}=i \int(d \xi) \gamma^{*}(\xi)(\delta / \delta q(\xi)) G^{*} .
$$

In order to evaluate the functional derivative in (9.5) and (9.6), we introduce the matrices $\Gamma(\xi)$ and $\Gamma^{*}(\xi)$ defined by

$$
\begin{gathered}
\Gamma(\xi)=-(\delta / \delta\langle\phi(\xi)\rangle) G^{-1}=G^{-1}\{\delta G / \delta\langle\phi(\xi)\rangle\} G^{-1}, \\
\Gamma^{*}(\xi)=-(\delta / \delta\langle\phi(\xi)\rangle) G^{*-1}=G^{*-1}\left\{\delta G^{*} / \delta\langle\phi(\xi)\rangle\right\} G^{*-1} .
\end{gathered}
$$

We then have the relation

$$
(\delta / \delta\langle\phi(\xi)\rangle) G=G \Gamma(\xi) G, \quad(\delta / \delta\langle\phi(\xi)\rangle) G^{*}=G^{*} \Gamma^{*}(\xi) G^{*},
$$

which give, using (9.4) and (9.6),

$$
\Gamma(\xi)=\gamma(\xi)+(\delta / \delta\langle\phi(\xi)\rangle) \Delta k, \quad \Gamma^{*}(\xi)=\gamma^{*}(\xi)+(\delta / \delta\langle\phi(\xi)\rangle) \Delta k^{*} .
$$


On the other hand, using (9.8) with (8.28),

$$
i\left(\delta / \delta q\left(\xi^{\prime}\right)\right) G=\int(d \xi) G \Gamma(\xi) G D\left(\xi-\xi^{\prime}\right), \quad i\left(\delta / \delta q\left(\xi^{\prime}\right)\right) G^{*}=\int(d \xi) G^{*} \Gamma^{*}(\xi) G^{*} D\left(\xi-\xi^{\prime}\right) .
$$

Therefore, substituting (9.10) in (9.5) and (9.6), we find that

$$
\Delta k=\iint(d \xi)\left(d \xi^{\prime}\right) \gamma\left(\xi^{\prime}\right) G \Gamma(\xi) D\left(\xi-\xi^{\prime}\right), \quad \Delta k^{*}=\iint(d \xi)\left(d \xi^{\prime}\right) \gamma^{*}\left(\xi^{\prime}\right) G^{*} \Gamma^{*}(\xi) D\left(\xi-\xi^{\prime}\right) .
$$

Further, using (9.11) in (9.9) together with (9.8), we get

$$
\begin{aligned}
\Gamma(\xi)=\gamma(\xi)+\iint\left(d \xi^{\prime}\right)\left(d \xi^{\prime \prime}\right) \gamma\left(\xi^{\prime}\right) G \Gamma(\xi) G \Gamma\left(\xi^{\prime \prime}\right) D\left(\xi^{\prime \prime}-\xi^{\prime}\right) \\
+\iint\left(d \xi^{\prime}\right)\left(d \xi^{\prime \prime}\right) \gamma\left(\xi^{\prime}\right) G\left\{\delta \Gamma\left(\xi^{\prime \prime}\right) / \delta\langle\phi(\xi)\rangle\right\} D\left(\xi^{\prime \prime}-\xi^{\prime}\right),
\end{aligned}
$$

and a similar equation result for $\Gamma^{*}(\xi)$.

The operators $\Delta k, \Delta k^{*}, \Gamma, \Gamma^{*}, G$, and $G^{*}$ can be constructed by successive approximation. Thus, to the first approximation, we find, using (9.11) and (9.12),

$$
\begin{aligned}
& \left\langle x|\Delta k| x^{\prime}\right\rangle \sim \gamma G_{0}\left(x-x^{\prime}\right) \gamma D\left(x-x^{\prime}\right), \\
& \left\langle x|\Gamma(\xi)| x^{\prime}\right\rangle \sim \gamma \delta(x-\xi) \delta\left(x-x^{\prime}\right)+\gamma G_{0}(x-\xi) \gamma G_{0}\left(\xi-x^{\prime}\right) \gamma D\left(x-x^{\prime}\right),
\end{aligned}
$$

where $G_{0}$ refers to the 0 th order approximation.

The Fourier transformation of the above equations in the limit $q=0$ are also important; in that case, because of the translation invariance, $G\left(x, x^{\prime}\right)=G\left(x-x^{\prime}\right),\left\langle x|\Delta k| x^{\prime}\right\rangle=\left\langle x-x^{\prime \prime}|\Delta k| x^{\prime}-x^{\prime \prime}\right\rangle$, etc., and also $\langle\phi(x)\rangle=0$. Thus, putting

$$
\begin{aligned}
& G(x)=\frac{1}{(2 \pi)^{3}} \int_{-\infty}^{\infty}(d t) e^{-i(t x)} G(t), \quad D(x)=\frac{1}{(2 \pi)^{3}} \int_{-\infty}^{\infty}(d t) e^{-i(t x)} D\left(t^{2}\right), \\
& \left\langle x|\Delta k| x^{\prime}\right\rangle=\frac{1}{(2 \pi)^{3}} \int_{-\infty}^{\infty}(d t) e^{-i\left(t\left(x-x^{\prime}\right)\right)} \Delta k(t), \\
& \left\langle x|\Gamma(\xi)| x^{\prime}\right\rangle=\frac{1}{(2 \pi)^{6}} \iint_{-\infty}^{\infty}\left(d t_{1}\right)\left(d t_{2}\right) e^{-i\left\{t_{1}(x-\xi)+t_{2}\left(x-x^{\prime}\right)\right\}} \Gamma\left(t_{1}, t_{2}\right)
\end{aligned}
$$

and the similar equation results for $G^{*}, \Delta k^{*}$, and $\Gamma^{*}$; then, from (9.4), (9.6), and (9.11),

$$
\left[(\gamma t)-k_{0}-\Delta k(t)\right] G(t)=1, \quad\left[-(\gamma t)-k_{0}^{*}-\Delta k^{*}(t)\right] G^{*}(t)=1,
$$

or

and

$$
G(t)=G_{0}(t)+G_{0}(t) \Delta k G(t), \quad G_{0}(t)=\left[(\gamma t)-k_{0}\right]^{-1},
$$

$$
\Delta k(t)=\frac{1}{(2 \pi)^{3}} \int_{-\infty}^{\infty}\left(d t^{\prime}\right) \gamma G\left(t+t^{\prime}\right) \Gamma\left(t^{\prime}, t\right) D\left(t^{\prime 2}\right) .
$$

The spectrum of possible waves in the fluctuating medium is given by the poles of $G(t)$, each pole of which gives the possible propagation constant.

In the actual situation, the correlation function between the waves at different points in space is very important, and it is thoroughly described by the Green's function introduced by (8.31). Thus, in view of (8.15),

$$
\operatorname{Av}\left[\psi_{\alpha}\left(x_{1}\right) \psi_{\beta}^{*}\left(x_{2}\right)\right]=\iint\left(d x_{1}^{\prime}\right)\left(d x_{2}^{\prime}\right) G_{\alpha \beta} ; \gamma_{\delta}\left(x_{1} ; x_{2} \mid x_{1}^{\prime} ; x_{2}^{\prime}\right) \eta \gamma\left(x_{1}^{\prime}\right) \eta_{\delta}^{*}\left(x_{2}^{\prime}\right)
$$


Here, the Green's function

$$
G\left(x_{1} ; x_{2} \mid x_{1}^{\prime} ; x_{2}^{\prime}\right) \equiv\left\langle x_{1}, x_{2}|G(1,2)| x_{1}^{\prime}, x_{2}^{\prime}\right\rangle
$$

satisfies the functional differential equation (8.34), which can also be represented by the following integro-differential equation corresponding to (9.4) and (9.6) (appendix 6), i.e.,

$$
F(1) F^{*}(2) G(1,2)-I(1,2) G(1,2)=1,
$$

where

$F(1)=\left[(\gamma p)-k_{0}-\Delta k-\int(d \xi) \gamma(\xi)\langle\boldsymbol{\phi}(\xi)\rangle\right]_{1}, \quad F^{*}(2)=\left[(\gamma p)-k_{0}^{*}-\Delta k^{*}-\int(d \xi) \gamma^{*}(\xi)\langle\boldsymbol{\phi}(\xi)\rangle\right]_{2}$

and the subscripts 1 and 2 refer to the coordinates $x_{1}$ and $x_{2}$, respectively. On comparison with (8.34), the interaction term $I(1,2)$ is found to be (appendix 6)

$$
I(1,2) G(1,2)=\iint(d \xi)\left(d \xi^{\prime}\right) \gamma_{1}(\xi) D\left(\xi-\xi^{\prime}\right) \Gamma_{2}^{*}\left(\xi^{\prime}\right) G(1,2)+i \int(d \xi) \gamma_{1}(\xi) G(1)(\delta / \delta q(\xi))\{I(1,2) G(1,2)\},
$$

which, to the first approximation, gives

$$
\left\langle x_{1}, x_{2}|I(1,2)| x_{1}^{\prime}, x_{2}^{\prime}\right\rangle \sim \gamma_{1} \gamma_{2}^{*} D\left(x_{1}-x_{2}\right) \delta\left(x_{1}-x_{1}^{\prime}\right) \delta\left(x_{2}-x_{2}^{\prime}\right) .
$$

The perturbation theory, as applied in the above, is not to be confused with the Born series expansion with respect to the coupling constant $\gamma$. As stated in part $\mathrm{I}$, it is equivalent to the selective summation of all the Feynman diagrams of the ladder type.

\section{Summary and Discussion}

The electromagnetic field distribution on an infinite plane $\sigma$ is completely described by the $\psi_{a}[\sigma]$ 's (or simply $\psi[\sigma]$ ) which are its components of a complete set of orthogonal modes of electromagnetic waves defined on the plane $\sigma$. When two infinite planes $\sigma_{1}$ and $\sigma_{2}$ are parallel to each other, $\psi\left[\sigma_{1}\right]$ is uniquely determined in terms of $\psi\left[\sigma_{2}\right]$ if the medium involved between $\sigma_{1}$ and $\sigma_{2}$ is given, while it may assume various values corresponding to a probability distribution function if the medium is fluctuating. Thus, the probability density function of waves is introduced (sec. 3). Here, the surfaces $\sigma_{1}$ and $\sigma_{2}$ may be concentric spherical surfaces having the same origin, or cylindrical surfaces having the same axis, since the existence of a complete set of orthogonal modes of waves on such surfaces is known [Stratton, 1941].

Also, an adjoint wave variable $\bar{\psi}[\sigma]$ is inevitably introduced to describe the statistical system, and both $\psi[\sigma]$ and $\bar{\psi}[\sigma]$ are represented by matrices or operators which operate on the probability density function of waves or, more generally, on the transformation function associated with it (sec. 5). These operators correspond to the canonical variables in field theory and satisfy the commutation relation of Bose statistics. Also, their spatial changes are prescribed by the same equation as the Heisenberg equation of motion in quantum mechanics. Further, the boundary condition of the wave (i.e., the outward propagating wave condition at infinity) is given in the same form as the vacuum condition in field theory.

In this connection, the $\psi[\sigma]$ seems to be more fundamental than the wave itself on the surface $\sigma$, since the former makes it possible to develop the whole theory as divergence-free, while it is not always true for the latter (sec. 2); not only can the matrix representations of $\psi[\sigma]$ and $\bar{\psi}[\sigma]$ be well defined on the surface of reference, but also the commutator exists between $\psi\left[\sigma_{1}\right]$ and $\bar{\psi}\left[\sigma_{2}\right]$ on different surfaces $\sigma_{1}$ and $\sigma_{2}$, while the matrix representation of the field $\psi(x)$ at a point $x$ is not determined uniquely unless otherwise the surface (on which the point $x$ exists) of reference is specified. Also, the commutator generally does not exist between $\psi\left(x_{1}\right)$ on $\sigma_{1}$ and $\bar{\psi}\left(x_{2}\right)$ on $\sigma_{2}$,

${ }^{16}$ Taking the limit of $q=0,(9.18)$ with (9.21) provides the equation (6.7) in part $\mathbf{I}$. 
although there exist some special matrix elements of the commutator. Excluding the above points, the analogies of the basic equations to those in field theory are rather remarkable, and many powerful methods known in the latter may be available, based on the fundamental relationships developed in this paper. For instance, the Feynman diagram technique in field theory is a consequence of the quantization, the Heisenberg equation of motion, and the boundary (vacuum) condition. Therefore, we could develop the corresponding technique, as discussed in part $\mathbb{I}$, by adopting the interaction representation for the wave variables, and also by using the boundary conditions specified by $\langle 0|$ and $|0\rangle$ of (8.14), i.e. (5.26b), (5.32), and (8.5). Also, the theory of statistical Green's functions can be developed almost in the same form as in the field theory. For instance, it may be noticed that the equation (9.18) takes the same form as the two particle Green's function in field theory, and therefore the methods used for solving the latter may be available to get the present Green's function in an appropriate approximation.

In this respect, the situation will be the same as in the recent theory of thermodynamic system consisting of many particles, where the theory has been rapidly developed after introducing the advanced techniques in field theory.

\section{Appendix 1}

Operating $1-\gamma_{3}^{2}$ on both sides of the wave equation (2.1), i.e.,

$$
(\gamma \partial) \psi=k \psi+\eta
$$

and using the algebraic relations $(2.2 \mathrm{~b})$ and also (2.18a), the left side of (A.1.1) becomes

$$
\left(1-\gamma_{3}^{2}\right)(\gamma \partial) \psi(x)=\left(1-\gamma_{3}^{2}\right)\left(\gamma \partial_{T}\right) \gamma_{3}^{2} \psi(x)=\left(1-\gamma_{3}^{2}\right)\left(\gamma \partial_{T}\right) \psi(\sigma(x))
$$

while the right side becomes

$$
\begin{aligned}
\left(1-\gamma_{3}^{2}\right)\{k \psi(x)+\eta(x)\} & =\left(1-\gamma_{3}^{2}\right)\left[k\left(1-\gamma_{3}^{2}\right) \psi(x)+k \gamma_{3}^{2} \psi(x)+\eta(x)\right] \\
& =k_{33} \psi(n(x))+\left(1-\gamma_{3}^{2}\right)\{k \psi(\sigma(x))+\eta(x)\}
\end{aligned}
$$

where

$$
\left(1-\gamma_{3}^{2}\right) k\left(1-\gamma_{3}^{2}\right)=\left(1-s_{3}^{2}\right) k\left(1-s_{3}^{2}\right)=k_{33}\left(1-s_{3}^{2}\right)
$$

since $s_{3}$ has the eigenvalue's $0, \pm 1$ and thus the only nonvanishing matrix element is the diagonal element corresponding to the eigenvector of $s_{3}=0$. Applying $\operatorname{Tr}^{\prime}$ on both sides of (A.1.4),

$$
k_{33}=\operatorname{Tr}^{\prime}\left[\left(1-s_{3}^{2}\right) k\right] \text {. }
$$

Thus, by equating (A. 1.2) and (A. 1.3),

$$
\psi(n(x))=k_{33}(x)^{-1}\left(1-\gamma_{3}^{2}\right)\left[\left\{\left(\gamma \partial_{T}\right)-k\right\} \psi(\sigma(x))-\eta(x)\right]
$$

\section{Appendix 2}

Using the wave equation (2.21) for $\cup_{a}\left(x,\left[\sigma_{2}\right]\right),(2.35)$ gives, on dropping the subscript 1 from $x_{1}$,

$$
\begin{aligned}
& {[(\gamma \partial)-k] \psi(x)=-[(\gamma \partial)-k]\left(1-\gamma_{3}^{2}\right) k_{33}(x)^{-1} \eta(x)} \\
& \quad+\gamma_{3} \cup_{a}(x,[\sigma]) N_{a b} \int_{\sigma} \psi_{b}^{*}\left(\sigma\left(x^{\prime}\right)\right)\left[1+\left\{-\left(\gamma \overleftarrow{\partial_{T}^{\prime}}\right)-k\left(x^{\prime}\right)\right\}\left(1-\gamma_{3}^{2}\right) k_{33}\left(x^{\prime}\right)^{-1}\right] \eta\left(x^{\prime}\right) d \sigma_{3}^{\prime},
\end{aligned}
$$

$\partial^{\prime}$ being the differentiation with respect to $x^{\prime}$ and $\sigma$ the surface containing the point $x$. Here, on 
referring to (2.33) and (2.34), the second term on the right side of (A.2.1) becomes

$$
\begin{aligned}
\gamma_{3}^{2} \int_{\sigma} \delta\left(\sigma(x)-\sigma\left(x^{\prime}\right)\right)\left[1+\left\{-\left(\overleftarrow{\gamma} \partial_{T}^{\prime}\right)-k\right\}\left(1-\gamma_{3}^{2}\right) k_{33}\left(x^{\prime}\right)^{-1}\right] \eta\left(x^{\prime}\right) d \sigma_{3}^{\prime} \\
=\gamma_{3}^{2} \eta(x)+\gamma_{3}^{2}\left\{\left(\gamma \partial_{T}\right)-k\right\}\left(1-\gamma_{3}^{2}\right) k_{33}(x)^{-1} \eta(x) .
\end{aligned}
$$

Hence, since $(\gamma \partial)\left(1-\gamma_{3}^{2}\right)=\left(\gamma \partial_{T}\right)\left(1-\gamma_{3}^{2}\right)$ in the first term on the right side of $(\mathrm{A} .2 .1)$, the right side of (A.2.1) becomes

$$
\gamma_{3}^{2} \eta(x)-\left(1-\gamma_{3}^{2}\right)\left\{\left(\gamma \partial_{T}\right)-k\right\}\left(1-\gamma_{3}^{2}\right) k_{33}(x)^{-1} \eta(x)=\eta(x),
$$

where, using (A.1.4), the following relations are used:

$$
\begin{aligned}
& \left(1-\gamma_{3}^{2}\right)\left(\gamma \partial_{T}\right)\left(1-\gamma_{3}^{2}\right)=0 \\
& \left(1-\gamma_{3}^{2}\right) k\left(1-\gamma_{3}^{2}\right)=\left(1-\gamma_{3}^{2}\right) k_{33}
\end{aligned}
$$

Thus the $\psi\left(x_{1}\right)$ given by (2.35) satisfies the given wave equation together with the boundary values on $\sigma_{2}$.

\section{Appendix 3}

Let $d a$ and $d b$ be complex numbers connected by $d b=e^{i \theta} d a$, then

$$
\begin{aligned}
& d b_{R}=\cos \theta d a_{R}-\sin \theta d a_{I}, \\
& d b_{I}=\sin \theta d a_{R}+\cos \theta d a_{I},
\end{aligned}
$$

or, in matrix form,

$$
\left(\begin{array}{l}
d b_{R} \\
d b_{I}
\end{array}\right)=\left(\begin{array}{lr}
\cos \theta-\sin \theta \\
\sin \theta & \cos \theta
\end{array}\right)\left(\begin{array}{l}
d a_{R} \\
d a_{I}
\end{array}\right)
$$

Hence,

$$
d b_{R} d b_{I}=d a_{R} d a_{I}, \text { or } \quad(d b)=(d a) .
$$

In the general case in which $d b=w d a$, it follows that

$$
(d b)=|w|^{2}(d a)
$$

When $d b$ is a complex vector having two components $\left(d b_{1}, d b_{2}\right)$ and connected with $d a=\left(d a_{1}\right.$, $\left.d a_{2}\right)$ by

$$
\left(\begin{array}{l}
d b_{1} \\
d b_{2}
\end{array}\right)=\left(\begin{array}{ll}
w_{11} & w_{12} \\
w_{21} & w_{22}
\end{array}\right)\left(\begin{array}{l}
d a_{1} \\
d a_{2}
\end{array}\right)
$$

each matrix element $w_{i j}$ should be considered as a two-rowed matrix as in (A.3.2). Thus, introducing the two-rowed matrices 1 and $\mathbb{I}$ defined by

$$
\mathbf{1}=\left(\begin{array}{ll}
1 & 0 \\
0 & 1
\end{array}\right), \quad \mathbf{I}=\left(\begin{array}{rr}
0 & -1 \\
1 & 0
\end{array}\right), \quad \mathbf{I}^{2}=-1
$$

it follows that any complex variable $w$ can be represented by

$$
w=w_{R} \mathbf{1}+w_{I} \mathbf{I}, \quad \operatorname{Det}(w)=w_{R}^{2}+w_{X}^{2}=|w|^{2} .
$$

Hence, the whole set of elements $w_{i j}$ can be represented in the space resulting from the direct product of the two independent spaces, one of which is spanned by 1 and $\mathbb{I}$. Therefore the total 
determinant of (A.3.5) can be obtained independently from the product of the determinants connected with the subspaces. Thus

$$
\left(d b_{1}\right)\left(d b_{2}\right)=\left|\operatorname{Det}\left(w_{i j}\right)\right|^{2}\left(d a_{1}\right)\left(d a_{2}\right)
$$

Here $\operatorname{Det}\left(w_{i j}\right)$ is the ordinary determinant with respect to the indices $i$ and $j$.

In the general case where

it follows that

$$
d b_{1} d b_{2} \ldots d b_{n}=\left|\frac{\partial\left(b_{1}, b_{2}, \ldots, b_{n}\right)}{\partial\left(a_{1}, a_{2}, \ldots, a_{n}\right)}\right| d a_{1} d a_{2} \ldots d a_{n}
$$

$$
\left(d b_{1}\right)\left(d b_{2}\right) \ldots\left(d b_{n}\right)=\left\|\frac{\partial\left(b_{1}, b_{2}, \ldots ., b_{n}\right)}{\partial\left(a_{1}, a_{2}, \ldots, a_{n}\right)}\right\|^{2}\left(d a_{1}\right)\left(d a_{2}\right) \ldots\left(d a_{n}\right) .
$$

\section{Appendix 4.}

In the first equation on the right side of (4.15), the first term becomes, using (4.16),

$$
\begin{aligned}
i \bar{\psi}\left[\sigma_{2}\right]^{\prime} N \psi\left[\sigma_{2}\right] & =i \bar{\psi}\left[\sigma_{2}\right]^{\prime} N\left\{\cup\left[\sigma_{2}, \sigma_{1}\right] N \psi\left[\sigma_{1}\right]^{\prime}+\int_{\sigma_{1}}^{\sigma_{2}} \cup\left[\sigma_{2}, \sigma\right] N \eta[\sigma] d x_{3}[\sigma]\right\} \\
& =i \bar{\psi}\left[\sigma_{1}\right] N \psi\left[\sigma_{1}\right]^{\prime}-i \int_{\sigma_{2}}^{\sigma_{1}} \bar{\psi}[\sigma] N \eta[\sigma] d x_{3}[\sigma]
\end{aligned}
$$

which gives the first and third terms of (4.14).

In the same way, in the second equation on the right side of (4.15),

$$
\begin{aligned}
-i \bar{\psi}\left[\sigma_{1}\right] N \psi\left[\sigma_{1}\right] & =-i \bar{\psi}\left[\sigma_{1}\right] N\left\{\cup\left[\sigma_{1}, \sigma_{2}\right] N \psi\left[\sigma_{2}\right]^{\prime}+\int_{\sigma_{2}}^{\sigma_{1}} \cup\left[\sigma_{1}, \sigma\right] N \eta[\sigma] d x_{3}[\sigma]\right\} \\
& =-i \bar{\psi}\left[\sigma_{2}\right]^{\prime} N \psi\left[\sigma_{2}\right]^{\prime}-i \int_{\sigma_{2}}^{\sigma_{1}} \bar{\psi}[\sigma] N \eta[\sigma] d x_{3}[\sigma],
\end{aligned}
$$

which gives the second and third terms of (4.14).

\section{Appendix 5}

Using the definition of $\psi^{ \pm}(x)$ of (8.3) together with (2.19a-c) and (2.39), (8.21) gives

$$
\begin{aligned}
& \left|\psi^{+}\left[\sigma_{2}\right]^{\prime}\right\rangle=\exp \left[-\frac{i}{2}\left\{\bar{\psi}_{b}^{-}\left[\sigma_{2}\right] N_{b a} \psi_{a}^{+}\left[\sigma_{2}\right]^{\prime}+\psi_{a}^{+*}\left[\sigma_{2}\right]^{\prime} N_{a b} \bar{\psi}_{b}^{-*}\left[\sigma_{2}\right]\right\}\right]\left|0^{+}\right\rangle \\
& \left\langle\psi^{-}\left[\sigma_{1}\right]^{\prime}\right|=\left\langle 0^{-}\right| \exp \left[\frac{i}{2}\left\{\bar{\psi}_{b}^{+}\left[\sigma_{1}\right] N_{b a} \psi_{a}^{-}\left[\sigma_{1}\right]^{\prime}+\psi_{a}^{-*}\left[\sigma_{1}\right]^{\prime} N_{a b} \bar{\psi}_{b}^{+*}\left[\sigma_{1}\right]\right\}\right]
\end{aligned}
$$

Hence, using the commutation relation (8.7) and the boundary condition (8.5), they are found to satisfy

$$
\begin{aligned}
& \psi_{a}^{+}\left[\sigma_{2}\right]\left|\psi^{+}\left[\sigma_{2}\right]^{\prime}\right\rangle=\psi_{a}^{+}\left[\sigma_{2}\right]^{\prime}\left|\psi^{+}\left[\sigma_{2}\right]^{\prime}\right\rangle, \\
& \bar{\psi}_{a}^{+}\left[\sigma_{2}\right]\left|\psi^{+}\left[\sigma_{2}\right]^{\prime}\right\rangle=0, \\
& \left\langle\psi^{-}\left[\sigma_{1}\right]^{\prime}\right| \psi_{a}^{-}\left[\sigma_{1}\right]=\left\langle\psi^{-}\left[\sigma_{1}\right]^{\prime}\right| \psi_{a}^{-}\left[\sigma_{1}\right]^{\prime}, \\
& \left\langle\psi^{-}\left[\sigma_{1}\right]^{\prime}\right| \bar{\psi}_{a}^{-}\left[\sigma_{1}\right]=0 .
\end{aligned}
$$


In view of (8.32),

\section{Appendix 6}

$$
\mathscr{F}(1) G(1,2)=G^{*}(2)
$$

Here $G^{*}(2)$ stands for the conjugate Green's function of the coordinates $x_{2}$ and $x_{2}^{\prime}$. Hence, using $F(1)$ and $F^{*}(2)$ defined by (9.19), and also (9.4) and (9.6), i.e.,

it follows that

$$
\text { i } F(1) G(1)=1, \quad F^{*}(2) G^{*}(2)=1
$$

$$
F^{*}(2) \mathscr{F}(1) G(1,2)=1, \quad F(1) \mathscr{F}^{*}(2) G(1,2)=1 .
$$

On the other hand, from (8.34) and (9.18),

$$
\begin{aligned}
& \mathscr{F}(1) \mathscr{F}^{*}(2) G(1,2)=1, \\
& I(1,2) G(1,2)=F(1) F^{*}(2) G(1,2)-1,
\end{aligned}
$$

which gives, together with (A.6.3),

$$
\begin{aligned}
\boldsymbol{I}(1,2) G(1,2) & =F(1)\left(F^{*}(2)-\mathscr{F}^{*}(2)\right) G(1,2) \\
& =(F(1)-\mathscr{F}(1))\left(F^{*}(2)-\mathscr{F}^{*}(2)\right) G(1,2)+\mathscr{F}(1)\left(F^{*}(2)-\mathscr{F}^{*}(2)\right) G(1,2),
\end{aligned}
$$

whose last term becomes

$$
\left[\tilde{F}(1), F^{*}(2)\right] G(1,2)+\left(F^{*}(2)-\widetilde{F} *(2)\right) \widetilde{F}(1) G(1,2),
$$

since $\mathscr{F}(1)$ and $\mathscr{F} *(2)$ commute; as noted below (8.34).

The last term of (A.6.6) vanishes, as is seen using (A.6.3) and the first equation of (A.6.4). Thus (A.6.5) becomes

$$
I(1,2) G(1,2)=(F(1)-\mathscr{F}(1))\left(F^{*}(2)-\mathscr{F}^{*}(2)\right) G(1,2)+\left[\mathscr{F}(1), F^{*}(2)\right] G(1,2) .
$$

Here, $F^{*}(2)=G^{*}(2)^{-1}$ from (A.6.2), and thus using (9.7),

$$
\begin{aligned}
{\left[\mathscr{F}(1), F^{*}(2)\right] } & =-i \int(d \xi) \gamma_{1}(\xi)\left\{(\delta / \delta q(\xi)) F^{*}(2)\right\} \\
& =i \iint(d \xi)\left(d \xi^{\prime}\right) \gamma_{1}(\xi) \Gamma_{2}^{*}\left(\xi^{\prime}\right)(\delta / \delta q(\xi))\left\langle\phi\left(\xi^{\prime}\right)\right\rangle \\
& =\iint(d \xi)\left(d \xi^{\prime}\right) \gamma_{1}(\xi) \Gamma_{2}^{*}\left(\xi^{\prime}\right) D\left(\xi-\xi^{\prime}\right)
\end{aligned}
$$

On the other hand, using the second equation of (A.6.5), the first term on the right side of (A.6.7) becomes, since $F(1)^{-1}=G(1)$ from (A.6.2),

$$
(F(1)-\mathscr{T}(1)) G(1) I(1,2) G(1,2)=\left\{-\Delta k_{1}+i \int(d \xi) \gamma_{1}(\xi)(\delta / \delta q(\xi))\right\}\{G(1) I(1,2) G(1,2)\},
$$

which, on account of (9.5), gives

$$
+i \int(d \xi) \gamma_{1}(\xi) G(1)(\delta / \delta q(\xi))\{I(1,2) G(1,2)\}
$$


Thus, (A.6.7) finally becomes

$$
I(1,2) G(1,2)=\iint(d \xi)\left(d \xi^{\prime}\right) \gamma_{1}(\xi) \Gamma_{2}^{*}\left(\xi^{\prime}\right) D\left(\xi-\xi^{\prime}\right) G(1,2)+i \int(d \xi) \gamma_{1}(\xi) G(1)(\delta / \delta q(\xi))\{I(1,2) G(1,2)\}
$$

In the same way, another expression is obtained in which the numbers 1 and 2 are interchanged.

\section{Appendix 7}

Let $f(x)$ be a complex function of a real variable $x$, defined in a finite range of $L \geqslant x \geqslant-L$, and $N$ be an integer, $N \geqslant 1$, and

$$
\Delta x=L / N, \quad x_{n}=n \Delta x, \quad n=0, \pm 1, \pm 2, \ldots, \pm N .
$$

Then, the set of values $f\left(x_{n}\right)(N-1 \geqslant n \geqslant-N)$ can be represented by

$$
f\left(x_{n}\right)=\sum_{m=-N}^{N-1}(2 L)^{-1 / 2} f_{m} e^{-i \pi m x_{n} / L}, \quad f\left(x_{-N}\right)=f\left(x_{N}\right),
$$

where

$$
f_{m}=\sum_{n=-N}^{N-1}(2 L)^{-1 / 2} \Delta x e^{i \pi m x_{n} / L f} f\left(x_{n}\right) .
$$

The proof can be obtained by substituting (A.7.3) into the right side of (A.7.2) and by using the identity

$$
\sum_{m=-N}^{N-1}(2 N)^{-1} e^{i \pi m\left(x_{n}-x_{l}\right) / L}=\sum_{m=-N}^{N-1}(2 N)^{-1} e^{i \pi m(n-l) / N}=\frac{\left(e^{-i \pi(n-l)}-e^{i \pi(n-l)}\right)}{2 N\left(1-e^{i \pi(n-l) / N}\right)}= \begin{cases}1 & n=l \\ 0 & n \neq l .\end{cases}
$$

In the limit for $N \rightarrow \infty$,

$$
\left.f_{m}\right|_{N_{\rightarrow \infty}}=(2 L)^{-1 / 2} \int_{-L}^{L} d x e^{i \pi m x / L f} f(x) .
$$

Thus, (A.7.3) corresponds to the Fourier transformation.

On the other hand, putting

$$
\begin{aligned}
\pi(m, n) & =\pi(n, m) \equiv(2 N)^{-1 / 2} e^{i \pi m x_{n} / L}=(2 N)^{-1 / 2} e^{i \pi m n / N}, \\
\pi^{*}(m, n) & =\pi(-m, n) ;
\end{aligned}
$$

$\pi^{*}(m, n)$ being the complex conjugate of $\pi(m, n),($ A.7.3) can alsn he represented by the alternative form

$$
f_{m}=\sum_{n=-N}^{N-1} \pi(m, n) f\left(x_{n}\right)(\Delta x)^{1 / 2},
$$

Here, in view of (A.7.4),

$$
\sum_{m=-N}^{N-1} \pi^{*}(n, m) \pi(m, l)=\delta_{n l} .
$$

Hence, according to (A.3.10), (A.7.7) gives

$$
\prod_{m=-N}^{N-1}\left(d f_{m}\right)=|\operatorname{Det}(\pi)|^{2} \prod_{n=-N}^{N-1}\left(d f\left(x_{n}\right)(\Delta x)^{1 / 2}\right),
$$

where, for a complex differential $d f$ having the real and imaginary parts $d f_{R}$ and $d f_{I}$, respectively, 
$(d f)$ is defined as

$$
(d f) \equiv d f_{R} d f_{I}
$$

while $\operatorname{Det}(\pi)$ is the determinant of the matrix $\pi(m, n)$ with respect to the indices $m$ and $n$.

On the other hand, it follows from (A.7.8) that

Thus, (A.7.9) can be reduced to

$$
|\operatorname{Det}(\pi)|^{2}=1 .
$$

$$
\prod_{m=-N}^{N-1}\left(d f_{m}\right)=\prod_{n=-N}^{N-1}\left(d f\left(x_{n}\right)(\Delta x)^{1 / 2}\right)
$$

In the limit $N \rightarrow \infty, \Delta x \rightarrow 0$ and thus the discrete set of values $f\left(x_{n}\right)$ 's tend to a continuous set of values when $f(x)$ is a continuous function of $x$, while the coefficients $f_{m}$ 's still remain as a discrete set of values in so far as the range $L \geqslant x \geqslant-L$ is bounded.

The result (A.7.12) is obtained for the general case of a complex function $f(x)$. When $f(x)$ is a real function, (A.7.12) is modified as follows: from (A.7.2), with the reference to (A.7.1),

$$
f\left(x_{n}\right)(\Delta x)^{1 / 2}=(2 N)^{-1 / 2}\left[\sum_{m=1}^{N-1}\left\{f_{m} e^{-i \pi m x_{n} / L}+f_{-m} e^{i \pi m x_{n} / L}\right\}+f_{0}+(-)^{n} f_{-N}\right],
$$

where, since $f\left(x_{n}\right)$ 's are real values,

$$
f_{-m}=f_{m}^{*}
$$

being the complex conjugate of $f_{m}$, and also the boundary values $f_{0}$ and $f_{-N}$ prove to be real values. Therefore, the real part of (A.7.13) becomes

$$
f\left(x_{n}\right)(\Delta x)^{1 / 2}=\sum_{m=0}^{N-1} \sum_{\nu=1}^{2} \cup\left(x_{n} \mid m, \nu\right) f_{m, \nu}
$$

where

and

$$
f_{m, \nu}=\left\{\begin{array}{ll}
\sqrt{2} f_{m R}, & \nu=1 \\
\sqrt{2} f_{m I}, & \nu=2,
\end{array} \quad(m \neq 0) \quad f_{0, \nu}= \begin{cases}f_{0}, & \nu=1 \\
f_{-N}, & \nu=2,\end{cases}\right.
$$

$$
\begin{aligned}
& \cup\left(x_{n} \mid m, \nu\right)=\cup^{T}\left(m, \nu \mid x_{n}\right)=\left\{\begin{array}{ll}
N^{-1 / 2} \cos \left(\pi m x_{n} / L\right), & \nu=1 \\
N^{-1 / 2} \sin \left(\pi m x_{n} / L\right), & \nu=2,
\end{array} \quad(m \neq 0)\right. \\
& \cup\left(x_{n} \mid 0, \nu\right)=\cup^{T}\left(0, \nu \mid x_{n}\right)= \begin{cases}(2 N)^{-1 / 2}, & \nu=1 \\
(2 N)^{-1 / 2}(-)^{n}, & \nu=2 .\end{cases}
\end{aligned}
$$

On the other hand, the real part of (A.7.4) can be given in the form

$$
\sum_{m=0}^{N-1} \sum_{\nu=1}^{2} \cup\left(x_{n} \mid m, \nu\right) \cup^{T}\left(m, \nu \mid x_{l}\right)=\delta_{n l} .
$$

Hence, the determinant of the matrix $U$ defined by (A.7.16) with respect to the indices $x_{n}$ and $(m, \nu)$ satisfies the relation

$$
\operatorname{Det}(U) \operatorname{Det}\left(U^{T}\right)=1 \text { or }\{\operatorname{Det}(U)\}^{2}=1 .
$$

Thus, from (A.7.14) and (A.7.15),

$$
\prod_{n=-N}^{N-1}\left\{d f\left(x_{n}\right)(\Delta x)^{1 / 2}\right\}=\prod_{m=0}^{N-1} \prod_{\nu=1}^{2} d f_{m, \nu}=\prod_{m=1}^{N-1}\left\{2\left(d f_{m}\right)\right\} \cdot d f_{0} d f_{-N} .
$$


If a bracket $\langle\mu[L] \mid \phi[L]\rangle$ is defined by

$$
\langle\mu[L] \mid \phi[L]\rangle=\exp \left[i \int_{-L}^{L} \mu(x) \phi(x) d x\right],
$$

as in (5.1la), it may be considered as the limit

$$
\lim _{N \rightarrow \infty} \exp \left[\sum_{n=-N}^{N-1} i\left\{\mu\left(x_{n}\right) \Delta x^{1 / 2}\right\}\left\{\phi\left(x_{n}\right) \Delta x^{1 / 2}\right\}\right] .
$$

Here, applying the representation (A.7.14) to $\mu\left(x_{n}\right)$ and $\phi\left(x_{n}\right)$ with (A.7.17), the expression (A.7.21) can be reduced to

$$
\langle\mu[L] \mid \phi[L]\rangle=\lim _{N \rightarrow \infty} \exp \left[i \sum_{m=0}^{N-1} \sum_{\nu=1}^{2} \mu_{m, \nu} \phi_{m, \nu}\right]
$$

Thus, $d \phi[L]$ can be defined by

$$
d \phi[L] \equiv \lim _{N \rightarrow \infty} \prod_{m=0}^{N-1} \prod_{\nu=1}^{2}\left\{(2 \pi)^{-1 / 2} d \phi_{m, \nu}\right\},
$$

which can also be represented as follows in view of (A.7.19):

$$
\lim _{. N \rightarrow \infty} \prod_{n=-N}^{N-1}\left\{d \phi\left(x_{n}\right)(\Delta x / 2 \pi)^{1 / 2}\right\} .
$$

A similar equation holds for $d \mu[L]$. Here, as is already noted, although the discrete sets of values $\phi\left(x_{n}\right)$ and $\mu\left(x_{n}\right)$ generally tend to continuous sets of values as $N \rightarrow \infty$, the $\phi_{m, \nu}$ 's and $\mu_{m, \nu}$ 's remain discrete in so far as the space $-L \leqslant x \leqslant L$ is bounded.

\section{References}

Alekseev, A. I. (1961), The application of the methods of quantum field theory in statistical physics, Soviet Phys. Uspekhi 4, 23.

Bardeen, J., L. N. Cooper, and J. R. Schrieffer (1957), Theory of superconductivity, Phys. Rev. $108,1175$.

Dyson, F. (1949a), The radiation theories of Tomonaga, Schwinger, and Feynman, Phys. Rev. 75, 486.

Dyson, F. (1949b), The $S$ matrix in quantum electrodynamics, Phys. Rev. 75, 1736.

Furutsu, K. (1963), On the statistical theory of electromagnetic waves in a fluctuating medium (I), J. Res. NBS 67D (Radio Prop.), No. 3, 303-323.

Lippmann, B. A., and J. Schwinger (1950), Variational principles for scattering processes (I), Phys. Rev. $79,469$.

Marcuvitz, N., and J. Schwinger (1951), On the representation of the electric and magnetic fields produced by currents and discontinuities in wave guides (I), J. Appl. Phys. 22, 806.

Matsubara, T. (1955), A new approach to quantum-statistical mechanics, Prog. Theoret. Phys. $14,351$.

Middleton (1960), Introduction to statistical communication theory, ch. 7 -sec. 7.3 (7.28), McGraw-Hill Book Co.

Martin, P. C., and J. Schwinger (1959), Theory of many-particle systems (I), Phys. Rev. $115,1342$.

Rickayzen, G. (1961), Scattering by impurities in metals, "Many-body problem," W. A. Benjamin, Inc., New York.

Schwinger, J. (1951), On the Green's functions of quantized fields, I, II, Proc. Natl. Acad. Sci. 37, $452 ; 455$.

Schwinger, J. (1951-1954), Theory of quantized fields I-VI, Phys. Rev. 82, 914; 91, 713; 91, 728; 92, 1283; 93, 615; 94, 1362.

Stratton, J. A. (1941), Electromagnetic theory, McGraw-Hill Book Co., New York.

U.S. GOVER NMENT PRINTING OFFICE . 1964 OL $-734-50$ 


UNITED STATES

GOVERNMENT PRINTING OFFICE DIVISION OF PUBLIC DOCUMENTS

WASHINGTON, D. C., 20402

OFFICIAL BUSINESS
PENALTY FOR PRIVATE USE TO AVOID PAYMENT OF POSTAGE. $\$ 300$

$$
\text { (GPO) }
$$






